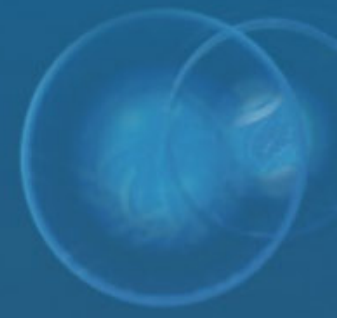

\title{
Journal of \\ Physical Science and Application
}

Volume 4, Number 2, February 2014

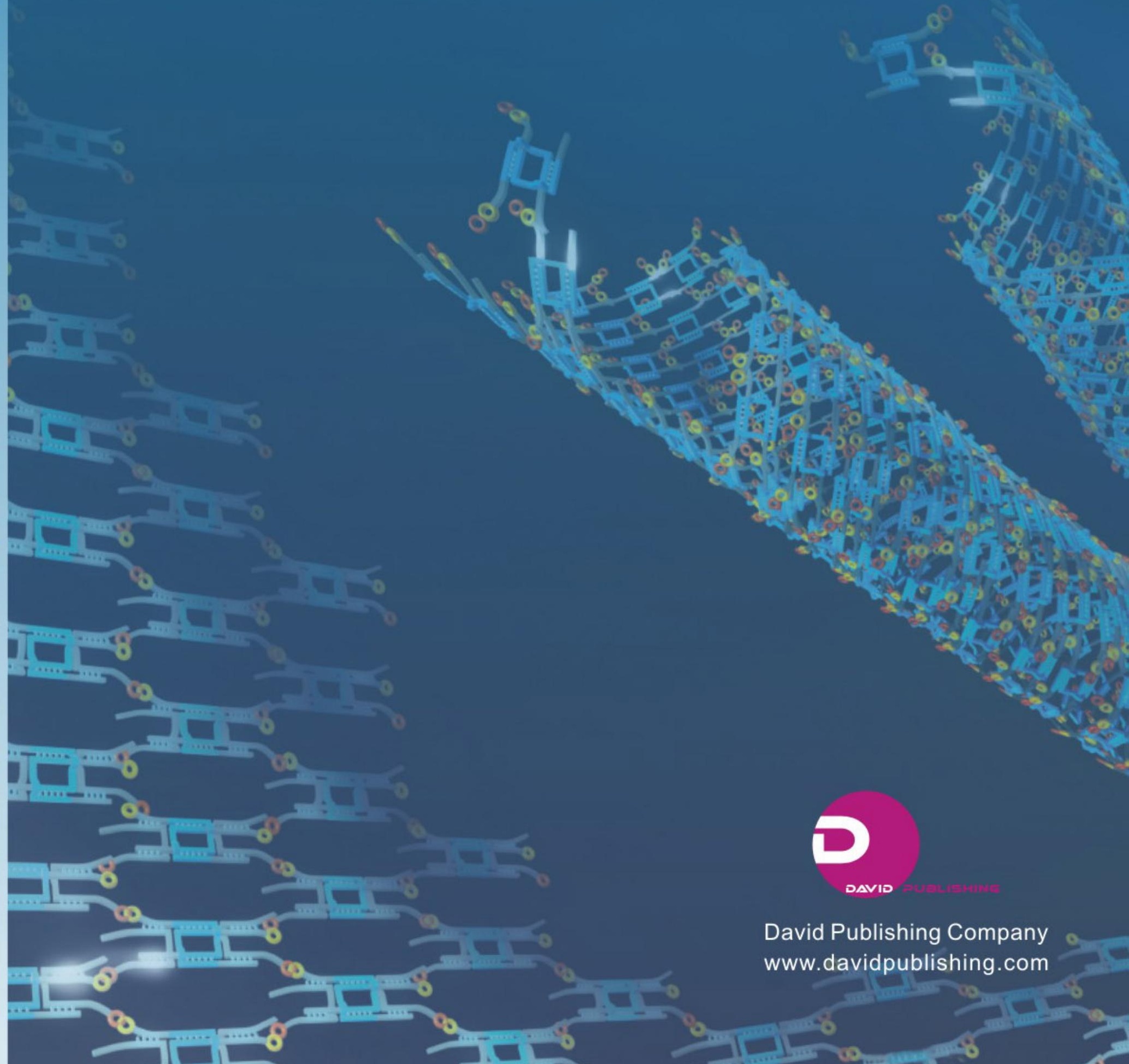




\section{Journal of Physical \\ Science and Application}

Volume 4, Number 2, February 2014 (Serial Number 19)

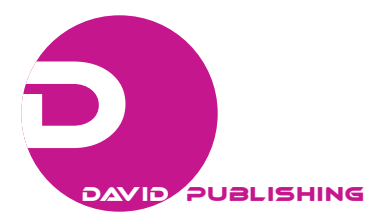

David Publishing Company

www.davidpublishing.com 


\title{
Publication Information:
}

Journal of Physical Science and Application is published monthly in hard copy (ISSN 2159-5348) by David Publishing Company located at 240 Nagle Avenue \#15C, New York, NY 10034, USA.

\begin{abstract}
Aims and Scope:
Journal of Physical Science and Application, a monthly professional academic journal, particularly emphasizes new medium for the publication of theoretical and experimental studies and reviews related to mechanics, acoustics, optical, magnetic, molecular principle, atomic theory, astrophysics, geophysics, biophysics, nuclear physics, chemical physics, and so on. Articles interpreting practical application of up-to-date technology are also welcome.
\end{abstract}

\section{Editorial Board Members:}

Prof. Aydin Mikail Ulubey (Italy), Prof. Damian Kachlakev (USA), Prof. A.N. Guz (Ukraine), Prof. Hesham Abdel Aziz Afifi (Egypt), Prof. Alejandro García Juárez (Mexico), Prof. Bagdoev A.G. (Armenia), Prof. Voronov Vladimir Kirillovich (Russian Federation), Prof. Tomasz Czujko (Poland), Assoc. Prof. Silvia Serranti (Roma), Dr. Elmar Christof Fuchs (Netherlands).

Manuscripts and correspondence are invited for publication. You can submit your papers via web submission, or E-mail to physical@davidpublishing.org; physics.david@yahoo.com. The submission guidelines and web submission system are available at http://www.davidpublishing.com.

\section{Editorial Office:}

240 Nagle Avenue \#15C, New York, NY 10034, USA

Tel: 1-323-984-7526, 323-410-1082

Fax: 1-323-984-7374, 323-908-0457

E-mail: physical@davidpublishing.org; physics.david@yahoo.com

Copyright@2014 by David Publishing Company and individual contributors. All rights reserved. David Publishing Company holds the exclusive copyright of all the contents of this journal. In accordance with the international convention, no part of this journal may be reproduced or transmitted by any media or publishing organs (including various websites) without the written permission of the copyright holder. Otherwise, any conduct would be considered as the violation of the copyright. The contents of this journal are available for any citation. However, all the citations should be clearly indicated with the title of this journal, serial number and the name of the author.

\author{
Abstracted/Indexed in: \\ Database of EBSCO, Massachusetts, USA \\ Cambridge Science Abstracts (CSA) \\ Ulrich's Periodicals Directory \\ Summon Serials Solutions \\ Chinese Database of CEPS, Airiti Inc. \& OCLC \\ Chinese Scientific Journals Database, VIP Corporation, Chongqing, China \\ Google Scholar (the published individual papers can be searched at: scholar.google.com) \\ ProQuest
}

\section{Subscription Information:}

Print \$450, Online \$320, Print and Online \$600 (per year)

David Publishing Company

240 Nagle Avenue \#15C, New York, NY 10034, USA

Tel: 1-323-984-7526, 323-410-1082; Fax: 1-323-984-7374, 323-908-0457

E-mail: order@davidpublishing.com

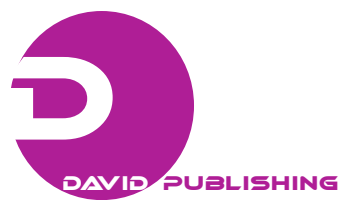

David Publishing Company

www.davidpublishing.com 


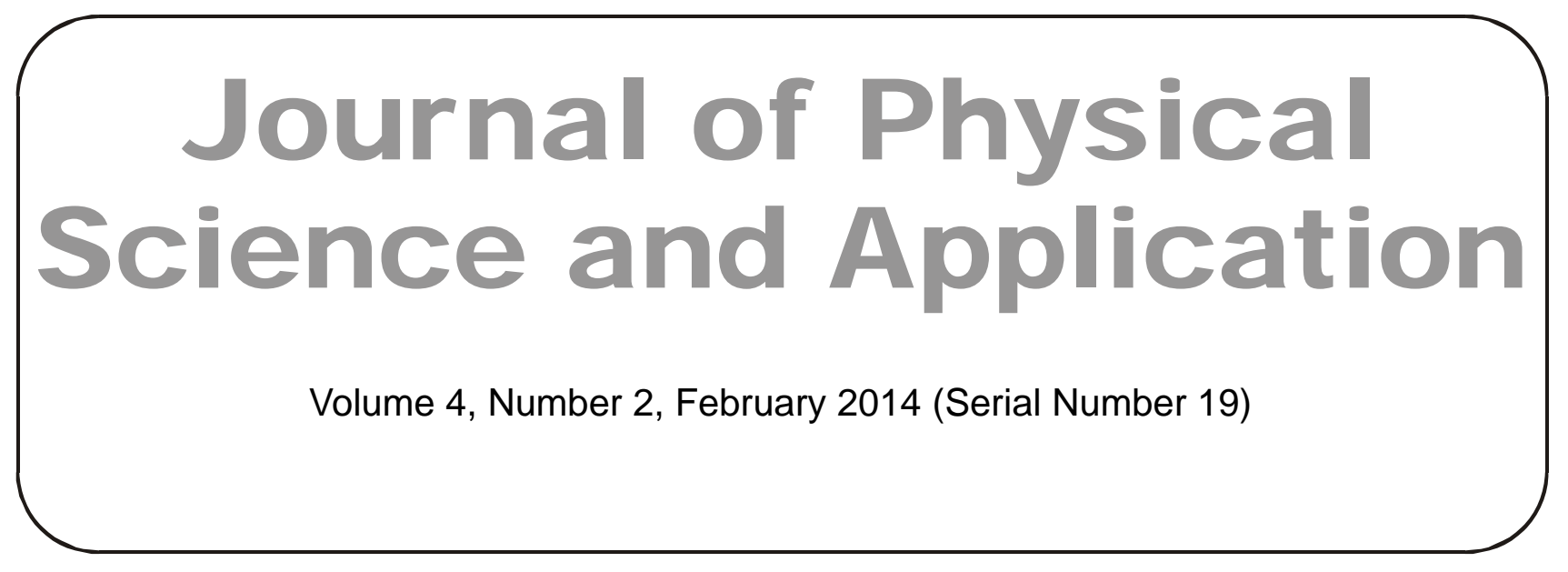

\section{Contents}

\section{Technological Studies}

71 Experimental Results on Excess Heat Power, Impurity Nuclides and X-Ray Production in Experiments with a High-Voltage Electric Discharge System

Alexander Karabut

84 Proposition of a New Type of Jointless System for Existing Concrete Bridges Yuichi Ishikawa, Minobu Aoyama, Masami Kuroyanagi, Masatugu Nagai and Takeshi Miyashita

90 The Parameters of the Stellarator as a Neutron Source for a Subcritical Reactor Vasiliy Rudakov

100 The Performance Analysis of the Wavelet-OFDM New Scheme in AWGN Channel Alaa Ghaith, Rima Hatoum and Hiba Mradand Ali Alaeddine

107 Enhancement of the Photodynamic Therapy Effect on Streptococcus Mutans Biofilm Vitor Hugo Panhóca, Mariana Carreira Geralde, Thaila Quatrini Corrêa, Mariana Torres Carvalho, Clóvis Wesley Oliveira Souza and Vanderlei Salvador Bagnato

115 Efficiency Improvement of 590 nm AlGaInP Light Emitting Diode with a Reflective Top Electrode Hyung Joo Lee, Young Dae Cho, Young Jin Kim, Choong Hun Lee, Jae Hoon Kim, Hwa Sub Oh and Su Chang Ahn

\section{Theoretical Studies}

119 Compensation Method: New Perspectives

Boris Levin

$130 \mathbf{H}^{ \pm}$Decays into $\mathbf{W}^{+}$and $\mathbf{a}_{1}$ in the Next Minimal Supersymmetric Standard Model Nguyen Chinh Cuong 


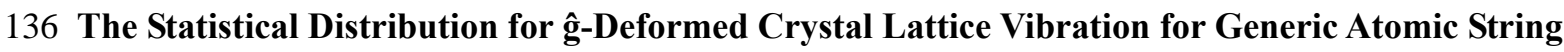
Nguyen Thi Ha Loan 


\title{
Experimental Results on Excess Heat Power, Impurity Nuclides and X-Ray Production in Experiments with a High-Voltage Electric Discharge System
}

\author{
Alexander Karabut \\ FSUE SIA LUTCH, Podolsk, Moscow Region 142100, Russia
}

Received: July 11, 2013 / Accepted: August 02, 2013 / Published: February 15, 2014.

\begin{abstract}
It reviewed results on low-energy nuclear reaction (LENR) processes in a high-voltage (1,000-4,000 V) electric discharge system. The experimental results are divided into three sets: excess heat measurements; yield of impurity nuclides (nuclear ash); X-ray measurements. Up to $8 \mathrm{~W}$ of excess power was observed, with a power gain of up to $170 \%$ was seen in glow discharge experiments. Up to $300 \mathrm{~W}$ of excess power, with a power gain up to $340 \%$ was observed in experiments using a high-voltage electrolysis cell. The impurity nuclide yield showing a shift of up to a few per cent from natural isotopic abundances was detected by spark mass spectrometry, by secondary ionic mass spectrometry, and by secondary neutral mass spectrometry. X-ray emission in the range of 0.6-6.0 keV, has been observed. Based on these experimental results we propose a phenomenological model for LENR based on the interaction of an electric discharge with condensed matter (of the cathode)
\end{abstract}

Key words: Electric discharge, excess heat, impurity nuclides, X-ray emission.

\section{Introduction}

Our group has studied low energy nuclear reactions (LENR) in glow discharge experiments for many years [1]. We have identified phenomena associated with the Fleischmann-Pons effect in the solid cathode of a high-voltage glow discharge system and a high-voltage electrolysis cell; including excess heat production, transmutation, and X-ray emission. In this work we will review experimental results on excess heat production in the glow discharge system (up to 8 $\mathrm{W}$, and $170 \%$ power gain) [2], and in the electrolysis cell (up to $300 \mathrm{~W}$, and $340 \%$ power gain) [3]. We also describe the results of experiments which show transmutation products [4]. Detailed experimental results on X-ray emission are discussed in another paper in the Ref. [5]. These include the observation of collimated X-ray emission from the cathode, which is a

Corresponding author: Alexander Karabut, Ph.D., doctor, research fields: plasma, laser and nuclear physics. E-mail: abkarab@mail.ru. new fundamental effect that has not been reported prior to our experiments.

Based on these observations, we have developed some theoretical ideas that may be helpful in understanding the experimental results. We propose that long-lived metastable states are generated at solid density in the cathode with excitation energies in the $\mathrm{keV}$ range. In the glow discharge, a flux of ions with kinetic energies on the order of $0.5-2.0 \mathrm{keV}$ is incident on the cathode; the appearance of X-rays in the 0.6-6.0 $\mathrm{keV}$ range implies that there is a physical mechanism capable of converting the incident ion energy into long-lived (up to tens of millisecond long) excitation of the electron-nuclear system of the cathode. We observe characteristic X-ray emission of the cathode elements, suggesting that $\mathrm{L}$ and $\mathrm{M}$ excitation occurs in the cathode. Observations of excess power and transmutation products indicates that nuclear reactions occur, and it may be that these reactions occur as a consequence of the high effective temperature that 

with a High-Voltage Electric Discharge System

might be associated with the strongly non-equilibrium conditions implied by the long-lived highly excited states. We propose that such reactions are responsible for LENR effects in our experiments.

\section{Experiments}

\subsection{Glow Discharge Device}

The measurements were carried out using a glow discharge [6] consisting of a water-cooled vacuum chamber, cathode and anode assemblies as shown in Fig. 1. The cathode holder can accept cathodes made of different metals, and the discharge occurs between the cathode and anode in the low pressure gas inside the chamber. Periodic pulses are supplied by a high-voltage power source. We used flow calorimetry, with independent water cooling channels supplied to the cathode, the anode, and to the chamber. Each cooling channel included two thermal sensors differently turned on at the input and output, and a cooling water flow meter.

\subsection{The High-Voltage Electrolysis Cell}

The measurements were carried out using highvoltage electrolysis cell; which consists of two flanges made of stainless still with fastening units for the anode and the cathode assembly as shown in Fig. 2 [7]. An electrolysis chamber in the form of quartz glass tube is set between the flanges, and another cylindrical tube set co-axially serves as the chamber cooling jacket. The electrolysis chamber volume was $95 \mathrm{~cm}^{3}$. The cathode units were provided with changeable cathode holders that allowed us to use different cathode samples with associated fasteners to hold the samples. The discharge chambers were wrapped up into a thermal insulation cover, witch reduced thermal losses into the environment. The three units of the device (the cathode, anode and chamber) had independent channels of water cooling. Each cooling channel included two temperature sensors (at the input and output) and a volumetric counter of the cooling water flow. The stirrer and the thermocouple were fixed upon the anode flange inside the chamber. $\mathrm{Pd}, \mathrm{Ni}$ and $\mathrm{Pt}$ cathode samples were used in the experiments; the cathode diameter was $11 \mathrm{~mm}$.

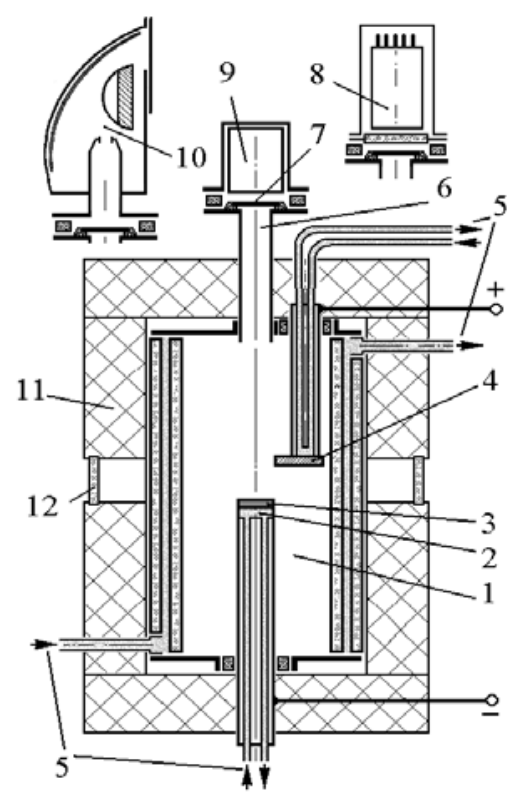

Fig. 1 Schematic representation of glow discharge device; (1) discharge chamber; (2) cathode holder; (3) cathode sample; (4) anode; (5) cooling water; (6) X-ray output; (7) Be foil screens; (8) X-ray scintillator, photomultiplier detectors. (9); $\mathrm{X}$-ray detectors different kind; (10) X-ray spectrometer; (11) heat insulation cover and (12) windows in heat insulation cover.

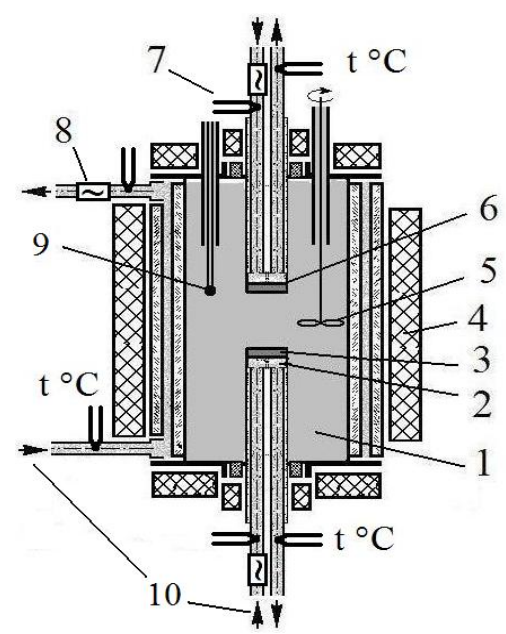

Fig. 2 Schematic of a high-voltage electrolysis device, set up as a heat capacity and flow calorimeter; (1) electrolyte chamber; (2) cathode holder; (3) cathode sample; (4) thermal insulation cover; (5) mixer; (6) anode; (7) temperature sensors; (8) flow meter; (9) thermocouple and (10) cooling water. 

with a High-Voltage Electric Discharge System

\subsection{Power Supply}

The power supply is designed to provide the glow discharge with periodic-pulse direct current, and permits the generation of desired current forms (of various pulse length and pulse period) to obtain the required current voltage operating conditions. The power supply consists of an autotransformer; a step-up transformer; a rectifier; a storage capacitor; a ballast resistor; and a high voltage transistor switch (Fig. 3). The power supply produces direct pulse-periodic current of rectangular shape of pulse. In the experiments, we used pulse duration from 0.1 to $2.0 \mathrm{~ms}$; and a period from 4 up to $100 \mathrm{~ms}$. The glow discharge conditions were: current (amplitude), from 30 up to $300 \mathrm{~mA}$; voltage, from 1,500 to $4,300 \mathrm{~V}$. The electrolysis voltage in different experiments ranged from $500 \mathrm{~V}$ to $2,500 \mathrm{~V}$, with the current in the range of 0.5-2.0 A.

\subsection{Excess Heat Power Measurements in Experiments}

The excess power parameters (determined from input from the temperature sensors and the flow-meter), and the electric parameters (the glow discharge current and voltage), were recorded using a data acquisition board. The excess heat power $\mathrm{P}_{\mathrm{EH}}$ was determined as the difference between the output thermal power and input electrical power. The discharge current and voltage

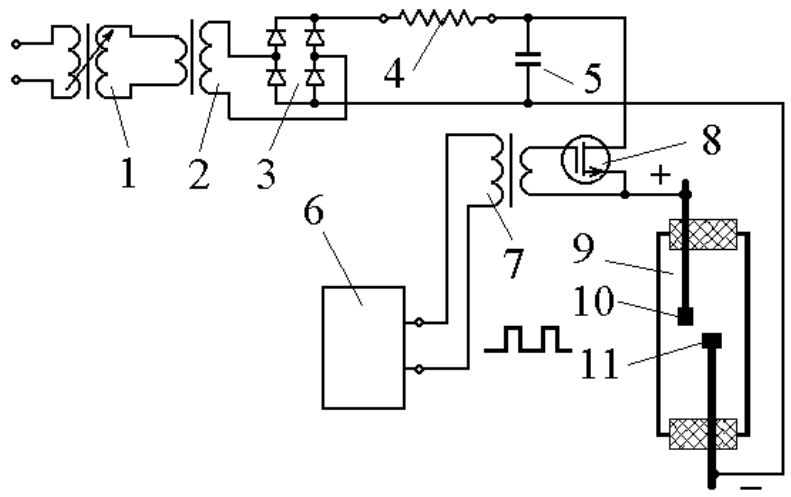

Fig. 3 Schematic of power supply; (1) autotransformer; (2) Step-up transformer; (3) rectifier; (4) resistor; (5) storage capacitor; (6) master generator; (7) guide transformer. (8) high-speed electronic switch based on fast IGBT transistors; (9) electric discharge chamber; (10) anode and (11) cathode. was measured with a two-channel data acquisition board. The use of 8-bit analog digital oscilloscope conversion (with $50 \mathrm{MHz}$ clock frequency) allowed us to achieve about $1 \%$ measurement accuracy of electric parameters. The electric power value having been obtained by the following expression:

$$
P_{e l}=1 / T \times \int U(t) I(t) d t
$$

Instantaneous current and voltage values were multiplied digitally and the resulting estimate for electric power was made; with the average electric power value obtained from We used a numerical integration based on

$$
P_{e l}=\frac{1}{T} \times \sum_{i=0}^{i=N} \frac{p_{i}+p_{i+1}}{2} \times\left(t_{i+1}-t_{i}\right)
$$

here, $T$ (the pulse-repetition period) is set by the power supply driving a generator with the accuracy no less than $0.1 \% ; p_{i}, p_{i+1}$ representing electric power values for different temporal meanings $\left(t_{i}, t_{i+1}\right)$. The number of partitions $i=N$ was determined by the condition of the power correct linear approximation for each length $t_{i}, t_{i+1}$. The calorimetric system actual error was defined in tests by a tailor-made resistive heater. The water-cooled flow heater in size was very near to an electric resistance, which in its turn was close to discharge resistance (about $5 \mathrm{k} \Omega$ and $1 \mathrm{k} \Omega$ ) and was characterized by its own small specific heat and adequate thermal insulation. In check experiments the heater was powered by the pulse-periodic power supply like Glow Discharge device and High Voltage Electrolysis Cell (HVEC). The procedure used in processing of results was the same as that used in HVEC experimentation. In so doing the electric power of calibration heaters was defined and measured. The temperature difference (in calibration) was determined by the formulae:

$$
\begin{aligned}
& \Delta T_{A}=P_{E I A} /\left(C_{w} \cdot G_{W A}\right) \\
& \Delta T_{C}=P_{E I C} /\left(C_{w} \cdot G_{W C}\right) \\
& \Delta T_{C h}=P_{E l C h} /\left(C_{w} \cdot G_{W C h}\right)
\end{aligned}
$$

here, $P_{E I A}, P_{E I C}, P_{E I C h}$ denote electric power (values) of calibration heaters in the anode, cathode and chamber water channels, respectively. 

with a High-Voltage Electric Discharge System

The heat power carried away by the anode, cathode and chamber cooling water channels, respectively, is determined in the following way:

$$
\begin{gathered}
P_{H A}=C_{w} \cdot G_{W A} \cdot \Delta T_{A} \\
P_{H C}=C_{w} \cdot G_{W C} \cdot \Delta T_{C} \\
P_{H C h}=C_{w} \cdot G_{W C h} \cdot \Delta T_{C h}
\end{gathered}
$$

where, $C_{w}$ stands for the water specific heat; $G_{W A}, G_{W C}$, $G_{W C h}$ show the anode, cathode and chamber cooling water flow rate, respectively; $\Delta T_{A}, \Delta T_{C}, \Delta T_{C h}$ denote the difference between the anode, cathode and chamber input and output cooling water temperature readings. The water flow rate is measured by volume flow meters with $\pm 0.5 \%$ relative measurement error.

With the availability of an excess heat power source $\left(P_{E H}\right)$ inside HVEC, its value is determined by the following:

$$
P_{E H}=\left(P_{H C}+P_{H A}+P_{H C h}\right)-P_{e l} \pm \Delta P_{\text {error }}
$$

The heat efficiency $\eta$ (the ratio between output heat power and the input electrical power) was determined by expression

$$
\eta=\left(P_{H C}+P_{H A}+P_{H C h}\right) / P_{e l}
$$

Testing of the system was carried out measurements in experiments with $\mathrm{Pt}$ cathode under the assumption that no there is excess heat power. Heat balance of the error information is $\pm 3 \%$ (Figs. $2 b$ and 12).

\section{Pre-Deuterated Pd Cathode Sample Preparation}

Bombardment by D2 ions in the glow discharge is viewed as the most effective procedure for loading deuterium into Pd [7]. The measurements were carried out using the glow discharge device consisting of a water-cooled vacuum chamber $\left(1,000 \mathrm{~cm}^{3}\right)$, and cathode and anode assemblies. The chamber design allowed the placement of cathode samples made of various materials on a water-cooled surface. The experiments were carried out using a high-current glow discharge in $\mathrm{D}_{2}$, with Pd samples. The power supply feeds the glow discharge with a periodic pulse direct current, and allows the generation of desired current forms (with various pulse length and pulse period) to obtain the required current voltage and current. We observed good $\mathrm{D}_{2}$ loading into Pd cathodes with this approach. The glow discharge current was from $30 \mathrm{~mA}$ up to $300 \mathrm{~mA}$, the voltage was from 500 to $1,400 \mathrm{~V}$, and the gas pressure in the chamber was between 1 and 25 Torr. The loading procedure can be summarized as:

(1) First pump the gas out of the discharge chamber;

(2) Bleed deuterium into the chamber until the pressure builds up to several Torr;

(3) Switch on the glow discharge and commence operation.

The $\mathrm{D}_{2}$ pressure is observed to decrease steadily when loading into the cathode Pd. Then the glow discharge is switched off, with D2 being pumped again into the chamber. The above procedure is repeated several times, the gas pressure being increased each time as shown in Fig. 4. During the experiment the $\mathrm{D} / \mathrm{Pd}$ ratio was determined by summing up the pressure drops.

\section{Excess Power Measurements in Glow Discharge Experiments}

The efficiency $\eta$ is determined in experiments under operational regimes in which excess heat is not observed. The Pd samples used in glow discharge calibration experiments with $\mathrm{Xe}$ and $\mathrm{Kr}$ were not pre-charged with deuterium, and relatively high values
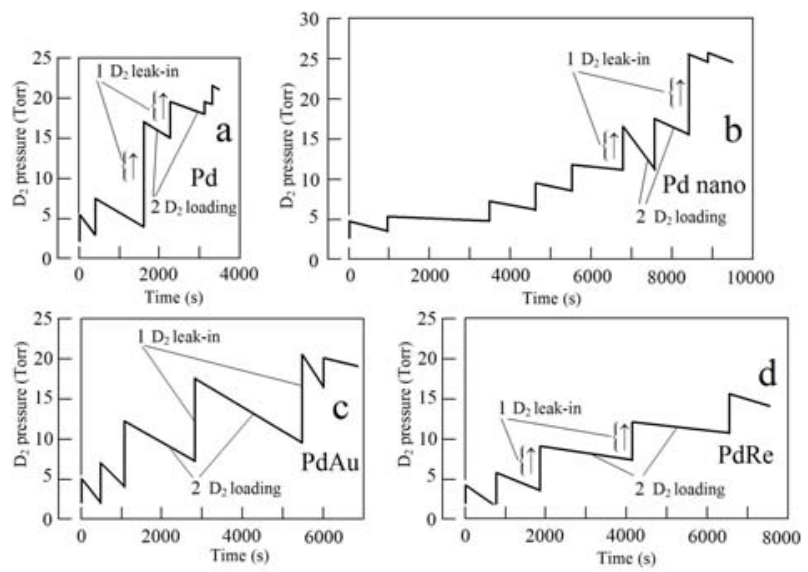

Fig. 4 Time history of the $D_{2}$ pressure in the glow discharge chamber; (a) $\mathrm{D}_{2}$ loading in Pd foil sample; (b) nanostructured Pd cathode sample, (c) Pd foil coated Au cathode sample and (d) Pd foil coated Re cathode sample. 

with a High-Voltage Electric Discharge System

for the thermal efficiency were achieved. Following calibration, deuterium is loaded into the Pd. In these experiments the current density did not exceed $100 \mathrm{~mA} / \mathrm{cm}^{2}$. At such values of the discharge current density in $\mathrm{D}_{2}$, a continuous loading of $\mathrm{D}_{2}$ into Pd ran up to saturation. The amount of deuterium loaded into palladium was determined by the volume of the gas absorbed from the discharge chamber. When saturation was achieved, the value of the $\mathrm{D} / \mathrm{Pd}$ ratio was close to 1 . Then, experiments were carried out with Pd cathode samples in $\mathrm{D}_{2}$, and also with deuterium pre-charged $\mathrm{Pd}$ cathode samples in $\mathrm{Xe}$ and $\mathrm{Kr}$ discharges. Relatively high values of excess power were achieved for deuterium pre-charged cathode samples in $\mathrm{Xe}$ and $\mathrm{Kr}$ discharges [7] (Fig. 5).

No excess power was observed in the cathode samples made of pure Pd (not deuterium pre-charged) in $\mathrm{Xe}$ and $\mathrm{Kr}$ discharges, as in Fig. 6. The biggest values of power gain were observed under conditions when the glow discharge voltage ranged between 1,000 and 1,300 V (Fig. 6). High values of the excess heat power were achieved for $\mathrm{Pd}$ deuterium pre-charged cathode samples in $\mathrm{D}_{2}, \mathrm{Xe}, \mathrm{Kr}$ discharges. No excess heat power production was observed in the cathode samples made of pure Pd (not deuterium pre-charged) in $\mathrm{Xe}$ and $\mathrm{Kr}$ discharges (Fig. 7).

We interpret this as showing two requirements for excess power generation: (1) deuterium should be loaded into the solid-state crystal lattice medium and (2) the crystal lattice should get an initial excitation, so that high-energy long-lived excited levels are created in the cathode sample solid. These excited conditions could be created by an additional source (for example by a flux of inert gas ions).

\section{Excess Power Measurements in the High-Voltage Electrolysis Cell (HVEC)}

The high-voltage electrolysis cell device was used in the two experimental runs:

(1) Experiments based on heat capacity calorimeter. The cooling water of the cathode, anode, and discharge chamber, was absent in these experiments;
(2) Experiments based on continuous flow calorimeter.

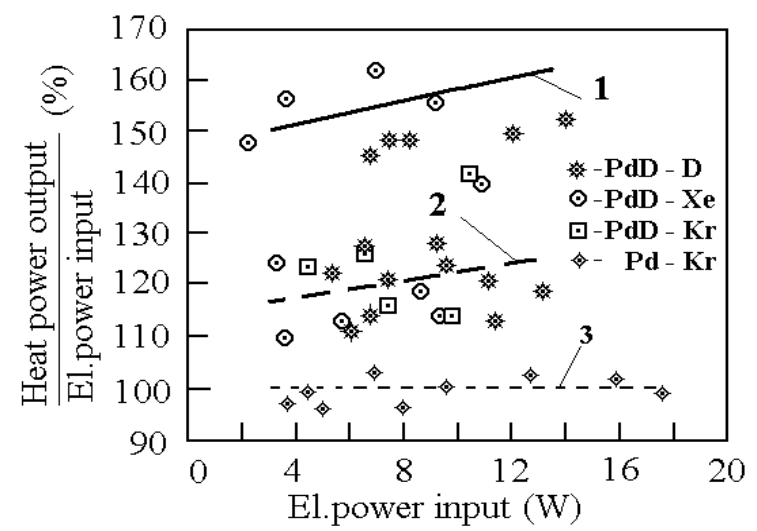

Fig. 5 Excess heat power a function of input electric power for a Pd cathode sample, $\mathrm{d}=9 \mathrm{~mm}$, current is 50-100 $\mathrm{mA}$. (1) Deuterium pre-charged $P d$ cathode samples in $D_{2} ; \mathrm{Xe}$, optimal (1,100-1,300 V) Glow Discharge voltage, (2) Deuterium pre-charged $P d$ cathode samples in $D_{2}$, Xe. Non-optimal (less 1,100 V) Glow Discharge voltage; (3) non-deuterium pre-charged $\mathrm{Pd}$ cathode in $\mathrm{Kr}$ discharge.

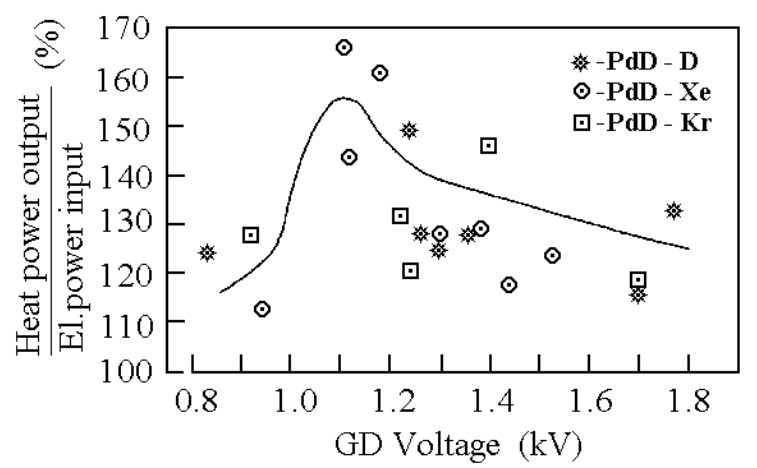

Fig. 6 The ratio of thermal power to electrical input power as a function of the glow discharge voltage. Deuterium pre-charged Pd cathode samples in $\mathrm{D}_{2}, \mathrm{Xe}, \mathrm{Kr}$ discharges.

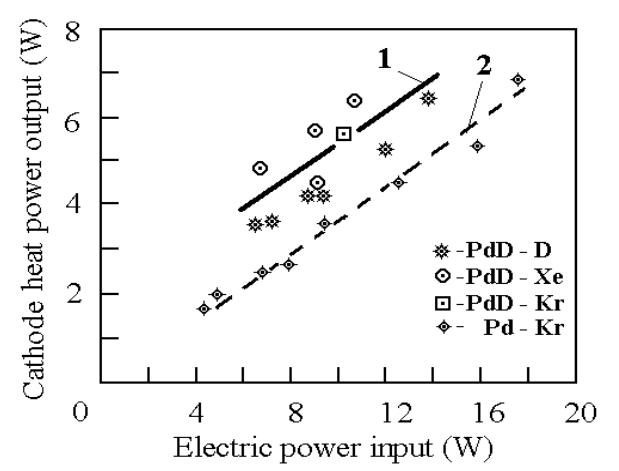

Fig. 7 Dependence of the cathode output heat power of the input electric power: (1) deuterium pre-charged Pd cathode samples in $D_{2}$, Xe and $K r$ discharges, $d=9 \mathrm{~mm}$, optimal $(1,100-1,300 \mathrm{~V})$ glow discharge voltage and (2) non deuterium-charged Pd cathode, in $\mathrm{Kr}$ discharge. 

with a High-Voltage Electric Discharge System

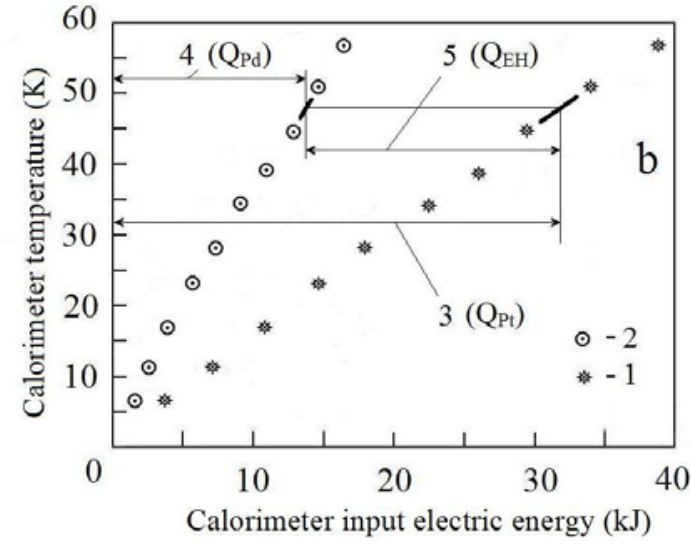

Fig. 8 Dependence of the calorimeter temperature up input electric energy value; (1) electrolysis in $\mathrm{H}_{2} \mathrm{O}$ with the $\mathrm{Pd}$ cathode, (2) electrolysis in $\mathrm{H}_{2} \mathrm{O}$ with a Pt cathode, (3) input electric energy in calorimeter with Pt cathode, (4) input electric energy in calorimeter with Pd cathode and (5) excess energy with pre-loaded Pd cathode.

\subsection{Heat Capacity Calorimetry in High-Voltage Electrolysis Cell Experiments}

A variety of calorimetry experiments were carried out in the high-voltage electrolysis cell to study excess power generation. For control experiments, we used $\mathrm{Pt}$ cathodes (which do not load, and which produce no excess power). Excess power measurements were made on Pd cathodes that were pre-loaded with deuterium, and also with Ni cathodes. The electrolysis was done in light water. In heat capacity calorimetry, estimation of the excess energy is made by comparing the temperature increase measured with deuterated $\mathrm{Pd}$ or $\mathrm{Ni}$ cathodes with the temperature rise of the $\mathrm{Pt}$ control. When a Pd cathode pre-loaded with deuterium is used, the temperature rise is much faster.To estimate the excess energy, we begin with the observed temperature as a function of input electrical energy; for each observed temperature we determine how much input energy is needed to produce such a temperature in the Pt control experiment, and then determine the excess energy as the difference between the two lines (the estimated energy produced minus the input electrical energy) Fig. 8. The electric discharge was switched off when the calorimeter temperature reached $57^{\circ} \mathrm{C}$. It is possible to develop estimates for the excess power produced from such measurements by determining the incremental excess energy produced during the time between successive temperature measurements. This approach was used for the estimated excess power in the different experiments of Figs. 9 and 10. We see from these measurements that the excess power produced reached $280 \mathrm{~W}$, with a power gain (ratio of thermal output power to electrical input power) as high as $340 \%$.
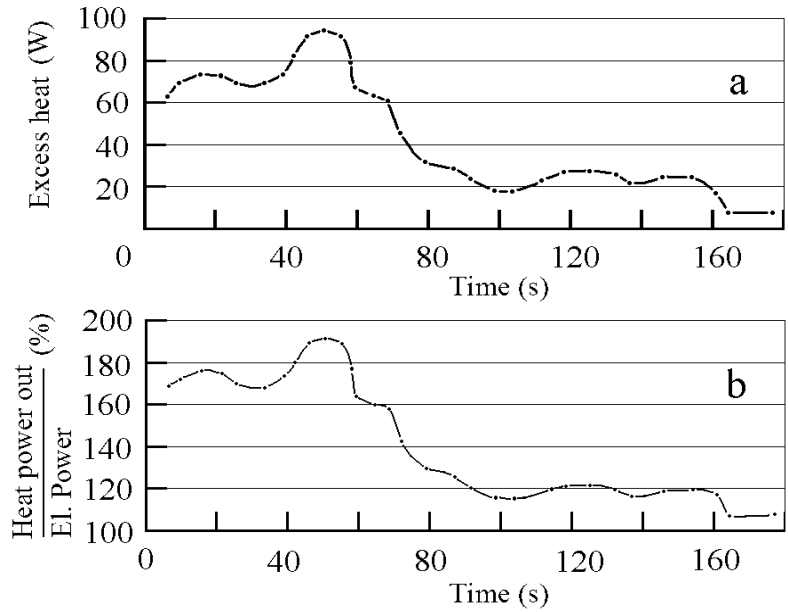

Fig. 9 (a) Excess power as a function of time and (b) power gain as a function of time. Electrolysis in $\mathrm{H}_{2} \mathrm{O}$ with a $\mathrm{Ni}$ cathode, cathode-anode voltage is $600 \mathrm{~V}$ and current is $0.7 \mathrm{~A}$.
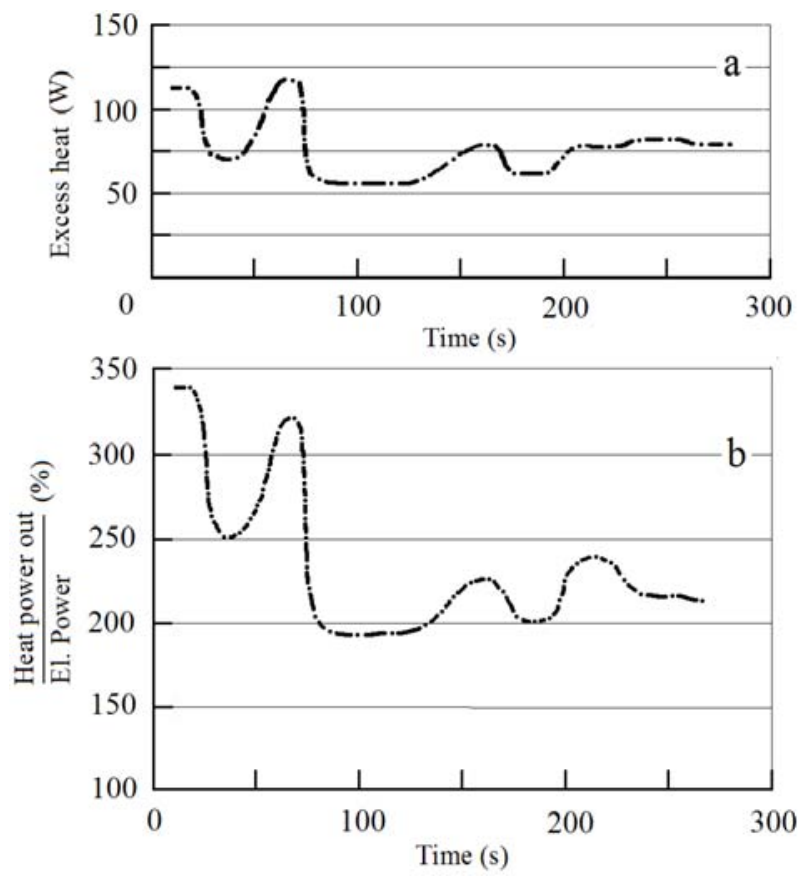

Fig. 10 (a) Excess power as a function of time and (b) power gain as a function of time. Electrolysis in $\mathrm{H}_{2} \mathrm{O}$ with a $\mathrm{D}_{2}$ pre-charged Pd cathode with nanostructure; cathode-anode voltage is $520 \mathrm{~V}$ and current is $2.1 \mathrm{~A}$. 

with a High-Voltage Electric Discharge System

The big values of Excess Heat power were registered for different kinds Pd cathode samples (Fig. 11) and Ni samples (Fig. 12).

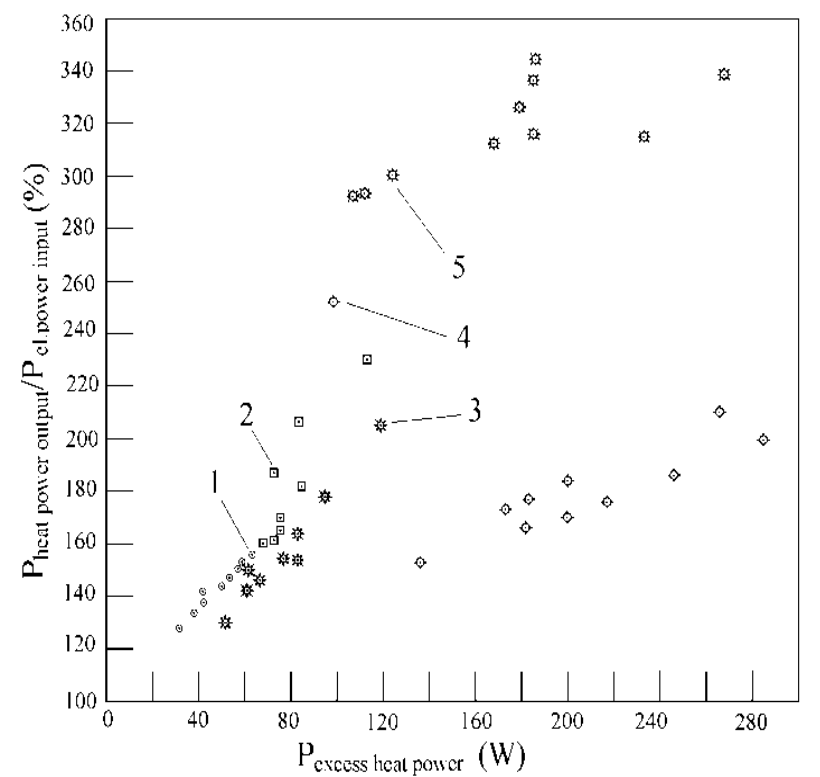

Fig. 11 Dependence of the output heat power and the input electric power ratio of the excess heat power from HVEC. $\mathrm{H}_{2} \mathrm{O}$ electrolyte. (1) Pd cathode sample, discharge voltage is 800-850 V, current is 0.6-0.7 A; (2) Pd coated Re, 650-700 V, 0.4-0.5 A; (3) nanostructure Pd, 1,000-1,200 V, 0.4-0.5 A; (4) nanostructure $P d, \quad 500-600 \quad V, \quad 1.8-2.0 \quad A$ and (5) nanostructure Pd, 500-600 V, 0.4-0.5 A.

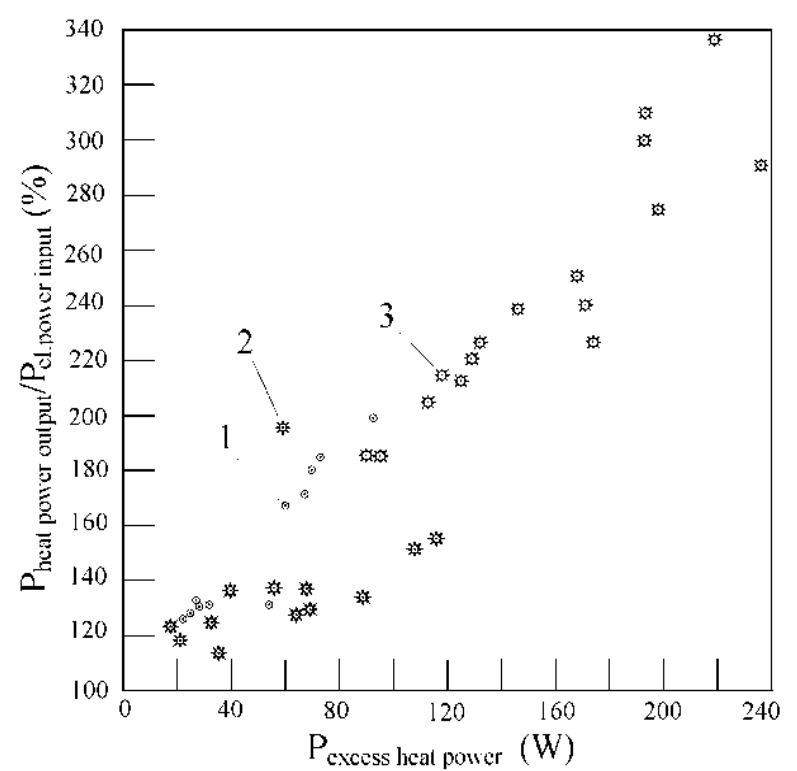

Fig. 12 Dependence of the output heat power and the input electric power ratio of the excess heat power from HVEC. $\mathrm{H}_{2} \mathrm{O}$ electrolyte: (1) Ni cathode sample, discharge voltage is $1,100-1,200 \mathrm{~V}$, current is $0.6-0.7 \mathrm{~A}$; (2) $\mathrm{Ni}$, 600-1,600 V; 0.4-0.5 $\mathrm{A}$ and (3) Ni, 1,200-1,300 V, 0.35-0.4 A.

\subsection{Flow Calorimetry in High-Voltage Electrolysis Cell Experiments}

Our flow calorimetry in the high-voltage electrolysis cell is similar to the calorimetry described above for the glow discharge experiments. Independent cooling water flow was channeled to the cathode, anode and to the chamber. Measurements of the temperature difference between the input and output of the different channels was used to determine the thermal power associated with the cathode, anode and chamber individually.

Experimental results are tabulated in Table 1 for $\mathrm{Pt}$ control experiments, and for experiments with $\mathrm{Ni}$ cathodes. No excess power was observed with $\mathrm{Pt}$ cathodes (Fig. 13), and the measured calorimeter efficiency was between $94 \%$ and $97 \%$. Excess power gain was seen with $\mathrm{Ni}$ cathodes with good reproducibility; the largest excess power observed was $230 \mathrm{~W}$ with a power gain of $280 \%$. The effect of the mixer (stirrer) can be seen in the greater proportion of power showing up in the chamber flow channel with the mixer on. Excess heat power is produced on cathode (Fig. 14).

\section{Impurity Nuclides Production Measurements}

\subsection{He Production Measurements}

Presently, the release of excess heat power is accounted for by ${ }^{4} \mathrm{He}$ production and the on-going reactions of transmutation accompanied by the impurity nuclides yield [8]. We had registered ${ }^{4} \mathrm{He}$ in the early experiments. Some of Pd cathodes together with the reference samples were analyzed at the Rockwell International Laboratory (Oliver's group). A small increase in the ${ }^{3} \mathrm{He}$ concentration and a large increase in the ${ }^{4} \mathrm{He}$ concentration was found in the discharge treated Pd samples. The results of two experiments represented in Table 2. This is the third independent evidence of ${ }^{4} \mathrm{He}$ presence in the nuclear reaction. 
Table 1 Flow calorimetry results for high-voltage electrolysis cell.

\begin{tabular}{|c|c|c|c|c|c|}
\hline \multicolumn{6}{|c|}{ Pt cathode, $\mathrm{H}_{2} \mathrm{O}$ electrolyte with working mixer } \\
\hline N Exp. & 1 & 2 & 3 & 4 & 5 \\
\hline Current* I (A) & 1.13 & 1.6 & 1.1 & 1.4 & 0.64 \\
\hline Voltage* U (V) & 600 & 443 & 295 & 380 & 1,520 \\
\hline Elec. Power $\mathrm{P}_{\mathrm{el}}(\mathrm{W})$ & 170 & 178 & 82 & 132 & 245 \\
\hline Cathode Heat power $\mathrm{P}_{\mathrm{HC}}(\mathrm{W})$ & 23.6 & 26.6 & 10.7 & 24.0 & 15.1 \\
\hline Chamber Heat power $\mathrm{P}_{\mathrm{HCh}}(\mathrm{W})$ & 124 & 128.5 & 57 & 100 & 145.4 \\
\hline Anode Heat power $\mathrm{P}_{\mathrm{HA}}(\mathrm{W})$ & 15.5 & 16.6 & 9.5 & 15.2 & 74.3 \\
\hline Total Heat power $\mathrm{P}_{\Sigma}(\mathrm{W})$ & 163 & 172 & 77.2 & 147 & 234 \\
\hline Excess Heat power $\mathrm{P}_{\mathrm{EH}}(\mathrm{W})$ & - & - & - & - & - \\
\hline Heat efficiency $\eta(\%)$ & 96 & 96 & 94 & 95 & 96 \\
\hline \multicolumn{6}{|c|}{$\mathrm{Ni}$ cathode, $\mathrm{H}_{2} \mathrm{O}$ electrolyte without working mixer } \\
\hline N Exp. & 1 & 2 & 3 & 4 & 5 \\
\hline Current* I (A) & 0.49 & 0.54 & 0.49 & 0.63 & 0.58 \\
\hline Voltage* U (V) & 1,500 & 1,900 & 1,700 & 1,460 & 1,770 \\
\hline Elec. Power $\mathrm{P}_{\mathrm{el}}(\mathrm{W})$ & 183 & 265 & 207 & 230 & 212 \\
\hline Cathode Heat power $\mathrm{P}_{\mathrm{HC}}(\mathrm{W})$ & 93.2 & 80.3 & 94.2 & 156.4 & 128.8 \\
\hline Chamber Heat power $\mathrm{P}_{\mathrm{HCh}}(\mathrm{W})$ & 128.3 & 199 & 198.3 & 165.6 & 127.6 \\
\hline Anode Heat power $\mathrm{P}_{\mathrm{HA}}(\mathrm{W})$ & 57.4 & 81 & 30.6 & 42.7 & 63.5 \\
\hline Total Heat power $\mathrm{P}_{\Sigma}(\mathrm{W})$ & 279 & 354 & 323 & 365 & 320 \\
\hline Excess Heat power $\mathrm{P}_{\mathrm{EH}}(\mathrm{W})$ & 88 & 89 & 116 & 137 & 108 \\
\hline Heat efficiency $\eta(\%)$ & 148 & 134 & 156 & 160 & 151 \\
\hline \multicolumn{6}{|c|}{$\mathrm{Ni}$ cathode, $\mathrm{H}_{2} \mathrm{O}$ electrolyte with working mixer } \\
\hline N Exp. & 1 & 2 & 3 & 4 & 5 \\
\hline Current* I (A) & 0.42 & 0.58 & 0.4 & 0.84 & 0.88 \\
\hline Voltage* U (V) & 1,110 & 1,070 & 620 & 654 & 634 \\
\hline Elec. Power $\mathrm{P}_{\mathrm{el}}(\mathrm{W})$ & 109 & 151 & 62 & 138 & 139 \\
\hline Cathode Heat power $\mathrm{P}_{\mathrm{HC}}(\mathrm{W})$ & 15.8 & 28.2 & 16.7 & 6.7 & 42.7 \\
\hline Chamber Heat power $\mathrm{P}_{\mathrm{HCh}}(\mathrm{W})$ & 113.5 & 162.5 & 93.8 & 286.5 & 268 \\
\hline Anode Heat power $\mathrm{P}_{\mathrm{HA}}(\mathrm{W})$ & 21 & 17 & 7.9 & 20.9 & 20.8 \\
\hline Total Heat power $\mathrm{P}_{\Sigma}(\mathrm{W})$ & 150 & 207.6 & 118 & 368 & 332 \\
\hline Excess Heat power $\mathrm{P}_{\mathrm{EH}}(\mathrm{W})$ & 40 & 69 & 56.3 & 230 & 180 \\
\hline Heat efficiency $\eta(\%)$ & 136 & 137 & 190 & 280 & 240 \\
\hline
\end{tabular}

*Current and voltage values was for pulse amplitude.

\subsection{Stable Impurity Nuclides Measurements}

We carried out elemental and isotopic assays of cathode sample before and after glow discharge experiments to search for possible nuclear reaction products. This was done using X-ray fluorescent spectrometry, spark mass spectrometry, secondary ionic mass spectrometry, and secondary neutral mass spectrometry. In the case of secondary ion mass spectrometry, the procedure that we used was:
(1) removal the upper $1.5 \mathrm{~nm}$-thick defect layer by plasma etching;

(2) scanning the first and the second layers in $5 \mathrm{~nm}$ increments, while determining the content of the impurity nuclides;

(3) removal of a layer with the thickness of $700 \mathrm{~nm}$, and repeated scanning of the third and fourth layers in 5 $\mathrm{nm}$ increments while again determining the content of the impurity nuclides (Fig. 15).

The initial impurity content was a few $10 \mathrm{ppm}$. We 

with a High-Voltage Electric Discharge System

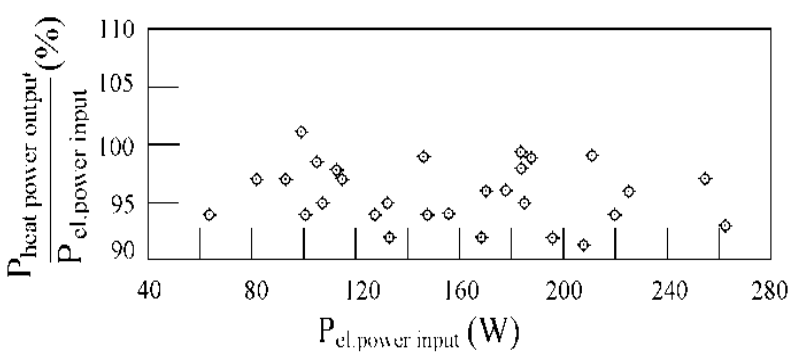

Fig. 13 The output heat power and the input electric power ratio for the different electric powers of HVEC discharge. Pt cathode sample.

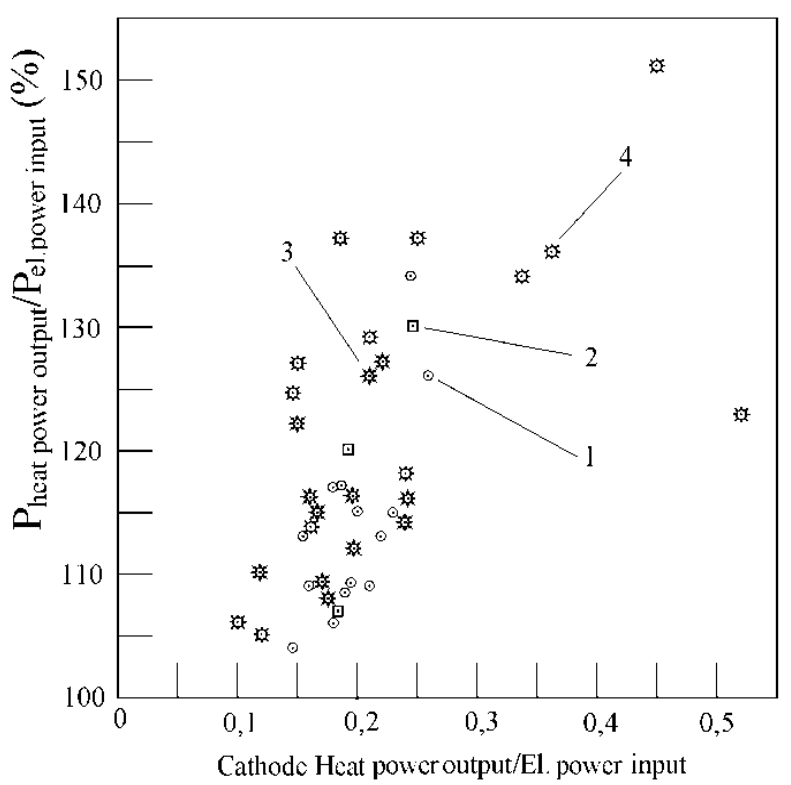

Fig. 14 Dependence of the output heat power and the input electric power ratio of the output cathode heat power and the HVEC input electric power ratio. (1) Ni cathode sample, discharge voltage is $600-1,800 \mathrm{~V}$, current is $0.6-0.9 \mathrm{~A}$. (2) $\mathrm{Ni}$, 500-700 V, 0.6-0.9 A. (3) nanostructure Pd, 700-1,700 V, 0.5-0.6 A.

Table 2 Relative content of ${ }^{3} \mathrm{He}$ and ${ }^{4} \mathrm{He}$ in $\mathrm{Pd}$ cathode samples from a glow discharge experiment. The cathode was Pd, the gas was $D_{2}$, the current was $35 \mathrm{~mA}$, and the experimental run time was $4 \mathrm{~h}$.

\begin{tabular}{lll}
\hline$\#$ & ${ }^{3} \mathrm{He}_{\text {after discharge }} /{ }^{3} \mathrm{He}_{\text {initial }}$ & ${ }^{4} \mathrm{He}_{\text {after discharge }} /{ }^{4} \mathrm{He}_{\text {initial }}$ \\
\hline 1 & up to 10 time & up to 100 time \\
2 & up to 2 time & up to 35 time \\
\hline
\end{tabular}

measured impurity nuclei with masses both less than, and greater than, that of Pd as depicted in Fig. 16. The main impurity elements (isotopes) with more than $1 \%$ content included: ${ }^{7} \mathrm{Li},{ }^{12} \mathrm{C},{ }^{15} \mathrm{~N},{ }^{20} \mathrm{Ne},{ }^{29} \mathrm{Si},{ }^{44} \mathrm{Ca},{ }^{48} \mathrm{Ca}$, ${ }^{56} \mathrm{Fe},{ }^{57} \mathrm{Fe},{ }^{59} \mathrm{Co},{ }^{64} \mathrm{Zn},{ }^{66} \mathrm{Zn},{ }^{75} \mathrm{As},{ }^{107} \mathrm{Ag},{ }^{109} \mathrm{Ag},{ }^{110} \mathrm{Cg}$, ${ }^{111} \mathrm{Cg},{ }^{112} \mathrm{Cg},{ }^{114} \mathrm{Cg},{ }^{115} \mathrm{In}$ (Table 3). The impurity content in the cathode sample volume was defined at different depths. The experiment duration in this case is up to $8 \times 10^{4} \mathrm{~s}$. The deviation from the natural isotope ratio for these impurity nuclei is up to several tens of times. Interestingly, the following isotopes were absent: ${ }^{58} \mathrm{Ni},{ }^{70} \mathrm{Ge},{ }^{73} \mathrm{Ge},{ }^{74} \mathrm{Ge},{ }^{113} \mathrm{Cd}$, and ${ }^{116} \mathrm{Cd}$, as shown in Fig. 17. These peculiarities are also registered within $1 \mu \mathrm{m}$ thick surface layer, the observed natural $\mathrm{Pd}$ isotopes ratio of the sample being changed (Fig. 18).

\section{X-Ray Measurements}

We have observed both diffuse and collimated X-ray

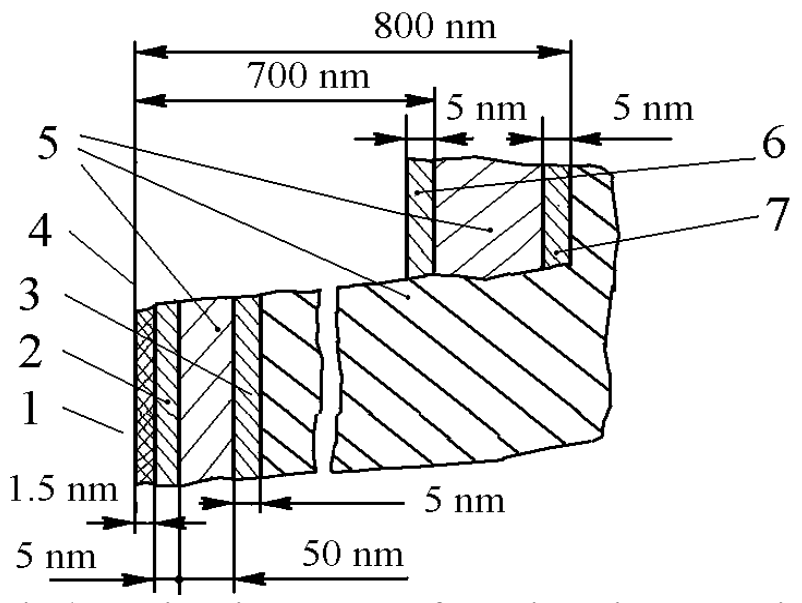

Fig. 15 Registration procedure for the impurity contents in the cathode samples by methods: Secondary Ion Mass Spectrometry (SIMS) and Secondary Neutral Mass Spectrometry (SNMS). (1) dirty superficial layer, (2), (3) analyzed layers (1), (2) scans, (4) surface of the cathode samples, (5) removal of a metal layer, (6), (7) analyzed layers, (3), (4) scans.

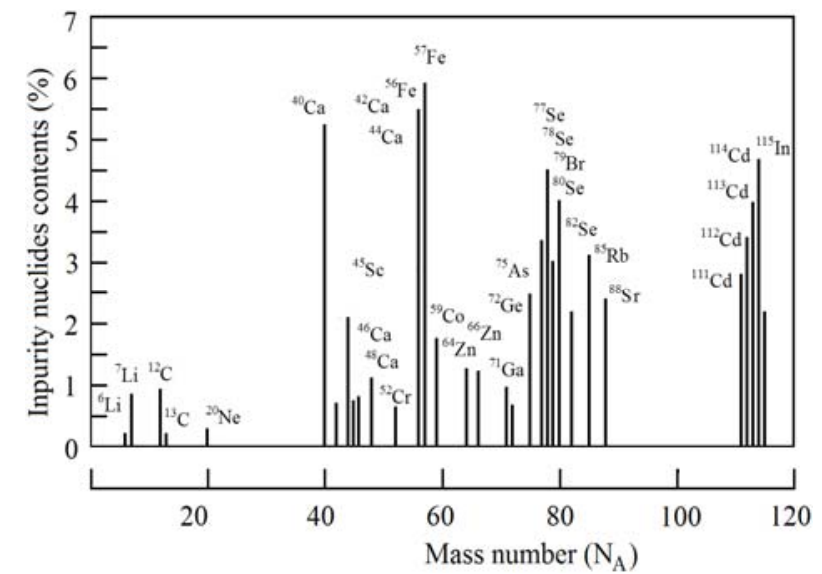

Fig. 16 Impurity nuclei content in the surface layer of the cathode sample (with a thickness of $100 \mu \mathrm{m}$ ) after glow discharge; $P d$ cathode, $D_{2}$ gas, $100 \mathrm{~mA}$ current, and $22 \mathrm{~h}$ run time. 

with a High-Voltage Electric Discharge System

Table 3 Stable impurity nuclides contents on different cathode sample depths after experiments.

\begin{tabular}{|c|c|c|c|c|}
\hline $\begin{array}{l}\text { A Impur. } \\
\text { nuclide }\end{array}$ & $\begin{array}{l}1 \mathrm{scan} \\
10 \mathrm{~nm}, \\
\text { content, \% }\end{array}$ & $\begin{array}{l}2 \mathrm{scan} \\
50 \mathrm{~nm}, \\
\text { content, \% }\end{array}$ & $\begin{array}{l}3 \text { scan } \\
700 \mathrm{~nm}, \\
\text { content, \% }\end{array}$ & $\begin{array}{l}4 \text { scan } \\
800 \mathrm{~nm}, \\
\text { content, } \%\end{array}$ \\
\hline${ }^{6} \mathrm{Li}$ & 0.075 & 0.22 & 0.21 & 0.16 \\
\hline${ }^{7} \mathrm{Li}$ & 0.84 & 0.53 & 0.45 & 0.47 \\
\hline${ }^{11} \mathrm{~B}$ & 0.14 & 0.31 & 0.18 & 0.18 \\
\hline${ }^{12} \mathrm{C}$ & 0.93 & 0.63 & 0.47 & 0.54 \\
\hline${ }^{13} \mathrm{C}$ & 0.19 & 0.15 & 0.05 & 0.06 \\
\hline${ }^{20} \mathrm{Ne}$ & 0.14 & 0.27 & 0.14 & 0.16 \\
\hline${ }^{42} \mathrm{Ca}$ & 0.72 & 1.14 & 1.08 & 0.8 \\
\hline${ }^{44} \mathrm{Ca}$ & 2.0 & 3.2 & 3.1 & 2.6 \\
\hline${ }^{45} \mathrm{Sc}$ & 0.74 & 0.91 & 0.86 & 0.8 \\
\hline${ }^{46} \mathrm{Ti}$ & 0.57 & 0.72 & 0.52 & 0.7 \\
\hline${ }^{47} \mathrm{Ti}$ & 0.25 & 0.14 & 0.31 & 0.14 \\
\hline${ }^{48} \mathrm{Ti}$ & 1.1 & 1.23 & 1.1 & 0.66 \\
\hline${ }^{52} \mathrm{Cr}$ & 0.62 & 0.41 & 0.31 & 0.1 \\
\hline${ }^{56} \mathrm{Fe}$ & 2.9 & 2.6 & 3.1 & 2.7 \\
\hline${ }^{57} \mathrm{Fe}$ & 5.5 & 3.25 & 3.53 & 3.16 \\
\hline${ }^{59} \mathrm{Co}$ & 1.0 & 1.0 & 1.4 & 1.5 \\
\hline${ }^{66} \mathrm{Zn}$ & 0.21 & 0.43 & 0.54 & 1.0 \\
\hline${ }^{71} \mathrm{Ga}$ & 4.0 & 4.9 & 5.6 & 3.4 \\
\hline${ }^{72} \mathrm{Ge}$ & 5.1 & 4.4 & 5.1 & 6.0 \\
\hline${ }^{75} \mathrm{As}$ & 6.2 & 4.9 & 7.4 & 4.7 \\
\hline${ }^{77} \mathrm{Se}$ & 3.4 & 3.9 & 4.8 & 4.0 \\
\hline${ }^{78} \mathrm{Se}$ & 4.5 & 3.45 & 5.8 & 1.4 \\
\hline${ }^{79} \mathrm{Br}$ & 3.0 & 2.4 & 2.8 & - \\
\hline${ }^{80} \mathrm{Se}$ & 4.0 & 3.4 & 2.5 & 2.3 \\
\hline${ }^{82} \mathrm{Se}$ & 3.4 & 3.0 & - & 3.2 \\
\hline${ }^{85} \mathrm{Rb}$ & 2.2 & 3.4 & 3.3 & 3.6 \\
\hline${ }^{88} \mathrm{Sr}$ & 3.1 & 4.4 & 4.2 & 6.0 \\
\hline${ }^{90} \mathrm{Zr}$ & 2.4 & 1.5 & 2.3 & 5.8 \\
\hline${ }^{111} \mathrm{Cd}$ & 2.8 & 3.0 & 3.0 & 3.4 \\
\hline${ }^{112} \mathrm{Cd}$ & 3.4 & 3.2 & 4.2 & - \\
\hline${ }^{113} \mathrm{Cd}$ & 4.0 & 1.8 & 2.8 & 5.1 \\
\hline${ }^{114} \mathrm{Cd}$ & 4.7 & 3.9 & 3.3 & 3.6 \\
\hline${ }^{115} \mathrm{In}$ & 2.2 & 2.5 & 2.3 & - \\
\hline
\end{tabular}

emission in the $\mathrm{keV}$ region in a large number of experiments carried out over the past decade; in this section we will provide a brief overview of some of our results [9]. Experiments were carried out using a glow discharge system, with a variety of X-ray diagnostics; including an X-ray pinhole camera, thermo-luminescent detectors with foils, scintillation detectors with a photomultiplier, and a curved mica spectrometer with X-ray film. Experiments were carried out on different metal cathode materials, including $\mathrm{Al}, \mathrm{Sc}, \mathrm{Ti}, \mathrm{Ni}, \mathrm{Nb}, \mathrm{Zr}, \mathrm{Mo}, \mathrm{Pd}, \mathrm{Ta}$, and $\mathrm{W}$. Different gasses were also used, including D2, H2, He, $\mathrm{Kr}$ and $\mathrm{Xe}$. The power supply delivered a train of pulses, with pulse durations between 0.1 and $2.0 \mathrm{~ms}$, and periods between $0.3 \mathrm{~ms}$ and $100 \mathrm{~ms}$. We used currents between 30 and $300 \mathrm{~mA}$, and voltages between 1,500 and $4,300 \mathrm{~V}$; the gas pressure in the chamber was 3-5 torr.

\subsection{Pinhole Camera Experiments}

The X-ray emission was of sufficiently high intensity that it was possible to image the cathode using a pinhole camera. We used a transverse $0.3 \mathrm{~T}$ magnetic field to verify that the image was not due to charged particles; it was observed that the image was nearly the same with and without the magnetic field.

\subsection{Thermo-Luminescent Detector Experiments}

We used crystalline $\mathrm{Al}_{2} \mathrm{O}_{3}$ thermo-luminescent

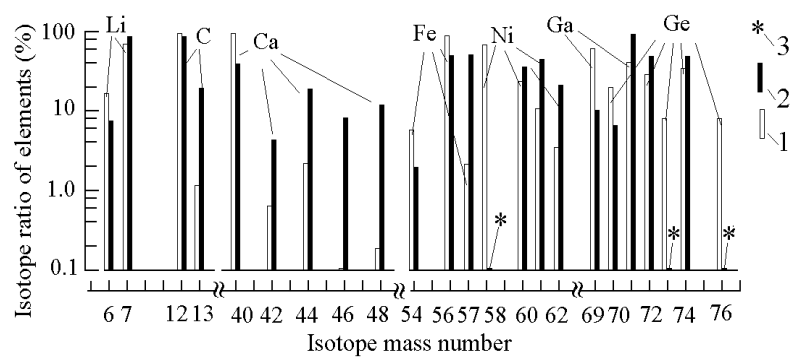

Fig. 17 Impurity nuclei ratio change in the surface layer of the cathode sample (with thickness of $100 \mu \mathrm{m}$ ) after glow discharge run; Pd cathode, $D_{2}$ gas, $100 \mathrm{~mA}$ current, and $22 \mathrm{~h}$ run time. (1) natural abundance, (2) post-run ratio, (3) absence of natural nuclei.

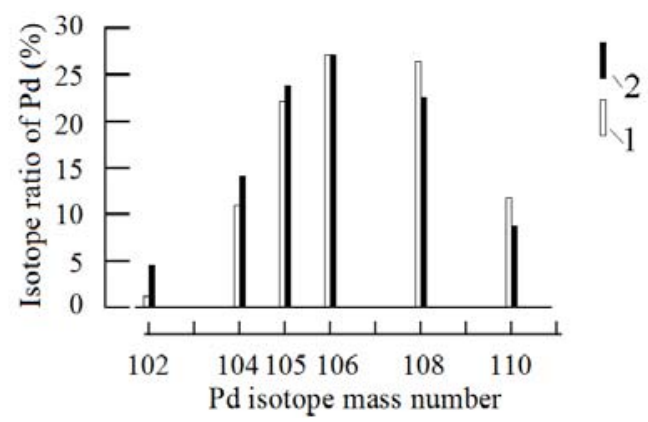

Fig. 18 Pd isotope ratio change in the surface layer of the cathode sample (with thickness of $100 \mu \mathrm{m}$ ) following a glow discharge run; Pd cathode, $D_{2}$ gas, $100 \mathrm{~mA}$ current, and $22 \mathrm{~h}$ run time. (1) natural ratio, (2) ratio after glow discharge. 
detectors covered with Be foils of different thicknesses in order to estimate the average energy of the X-ray emission [9]. We found that the main component was between $1.3 \mathrm{keV}$ and $1.8 \mathrm{keV}$, with evidence of a higher energy component as well. These measurements showed that the X-ray intensity from the cathode surface increased exponentially with an increase in the discharge voltage, reaching as upper limit of $1 \mathrm{~W}$.

\subsection{Scintillator and Photomultiplier Experiments}

We made use of organic with day (PMMA) scintillators with the optical scintillation detected using a photomultiplier and scope, to study the energy-dependence, spatial-dependence, and the time-dependence of the X-ray emission [9]. Tests with a transverse $0.3 \mathrm{~T}$ magnetic field again showed that the radiation was not composed of charged particles. We measured the transmission through $15-30 \mu \mathrm{m}$ thick Be foils, and the difference in transmission was consistent with an average energy in the range of $1.0-2.5 \mathrm{keV}$. It was found that the average energy for different cathode materials in these measurements were in good agreement with the thermo-luminescent detector measurements discussed above. We also did time-resolved measurements which showed that the diffuse radiation was produced while the discharge was on, and collimated emission was observed in bursts that followed the switching off of the discharge with some delay.

\subsection{Curved Mica Spectrometer Experiments}

Many experiments were carried out with the curved mica crystal spectrometer to give energy-resolved X-ray spectra on X-ray film [9]. A variety of spectral features were observed. We saw characteristic X-ray emission from $\mathrm{Kr}$ and $\mathrm{Xe}$, which showed up as strong spectral lines on the film. We also saw weaker characteristic X-ray emission from transitions in the host metal atoms. There was a continuum apparent that originated from the cathodes surface, centered roughly near $1.5 \mathrm{keV}$, with somewhat different widths depending on the discharge voltage, and with an intensity that was weakly correlated with the cathode material. An example of this continuum emission is shown in Fig. 19. Finally, we saw extremely strong micro-beam emission that was sufficiently intense to cause a bleaching (the proper technical term is solarization) of the film, and which appeared on the film as spots (instead of as spectral lines) and curves (consistent with a spot which moved during the emission).

\section{Interpretation and Proposed Physical Mechanisms}

Based on the experimental results reviewed in the previous sections, we can consider possible physical processes that might be involved. Presumably, some excited energetic levels are formed in the cathode solid when its surface is exposed to bombardment by the ions flux generated in plasma or electrolyte medium. It would follow that the observed X-ray emission occurs as a result of de-excitation of these energetic levels.

Deuterium ion acceleration in the glow discharge near-cathode region produced ion energies from several hundred $\mathrm{eV}$ up to a few thousand $\mathrm{eV}$.

Deuterium ions collide with the atoms of the crystal lattice ions. Presumably, some long-lived excited levels with energies up to several kilovolts are formed in the cathode solid. Under the resulting highly non-equilibrium conditions there exists an excited state

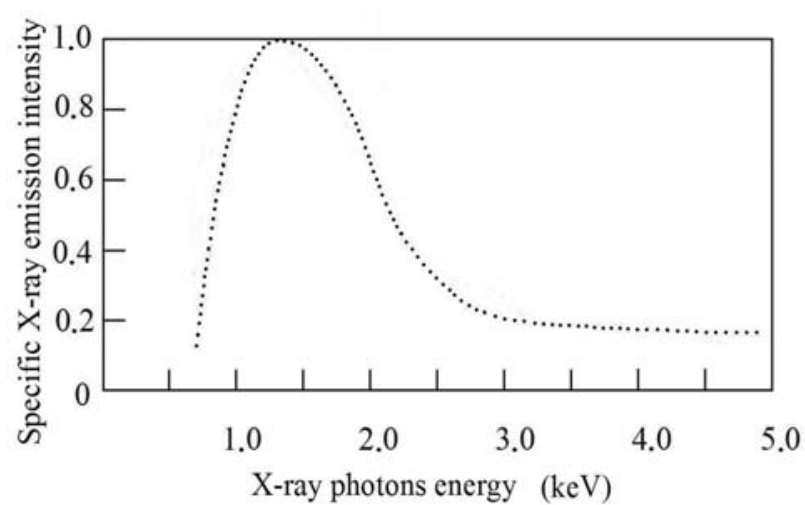

Fig. 19 Continuum X-ray emission measured with the curved mica crystal spectrometer from a glow discharge experiment with a Pd cathode and $\mathrm{D}_{2}$ gas. 

with a High-Voltage Electric Discharge System

population that can be characterized by an effective temperature on the order of $1-3 \mathrm{keV}$, which in our view are the conditions necessary for low-energy nuclear reactions to occur. Within this framework, we can think about what kinds of reactions in particular might lead to the formation of stable transmutation products.

The following reactions may be possible:

(1) $\mathrm{Pd}+\mathrm{mD} \rightarrow[\mathrm{PdmD}]^{*}$

(2) $[\mathrm{PdmD}]^{*} \rightarrow \mathrm{Pd}^{*}+{ }^{4} \mathrm{He}+$ Heat

(3) $[\mathrm{PdmD}]^{*} \rightarrow \mathrm{A}^{*}+\mathrm{B}^{*} \rightarrow \mathrm{A}+\mathrm{B}+$ Heat

(4) $[\mathrm{PdmD}]^{*} \rightarrow \mathrm{C}+$ Heat

where $[\mathrm{PdmD}]^{*}$ is a short-lived intermediate compound nucleus; $\mathrm{m}=1,2,3 \ldots ; \mathrm{A}^{*}, \mathrm{~B}^{*}$ denote nuclear isomers of nuclei with masses less than that of Pd; where A, B are stable nuclides; and where C stands for a nuclide with a mass more than that of Pd. The first step in the proposed process is the formation of an excited state compound-nucleus. Then one of three possible modes is realized:

(1) The compound nucleus may lose its excitation and form an exited Pd nucleus and ${ }^{4} \mathrm{He}$;

(2) The compound nucleus may split into two nuclear fragments with masses less than that of Pd. In so doing the two nuclei should be in excited isomer state (experiments show that the nuclear reactions energy is not produced as kinetic energy of nuclear fragments);

(3) The compound nucleus may lose its excitation and form a stable nucleus of a heavier than Pd element.

To determine the specific physical mechanism for these reactions will require additional research. One possible type of reaction for forming the impurity nuclides can be a long-range (resonant) nuclear reaction. The mechanism of such long-range reactions can be explained using as an example a specific transmutation reaction for Pd $+\mathrm{D}$ (Fig. 20a) and Pd + 2D (Fig. 20b) [9]. The formation of significant ${ }^{13} \mathrm{C}$ nuclei and ${ }^{93} \mathrm{Nb}$ nuclei was recorded in the experiments. In this case we assume that the reaction can proceed as

${ }^{104} \mathrm{Pd}+2 \mathrm{D} \rightarrow{ }^{106}[\mathrm{Pd} ; \mathrm{D}] \rightarrow{ }^{13} \mathrm{C}+{ }^{93} \mathrm{Nb}+7.820 \mathrm{MeV}$ Momentum and energy conservation dictates that the

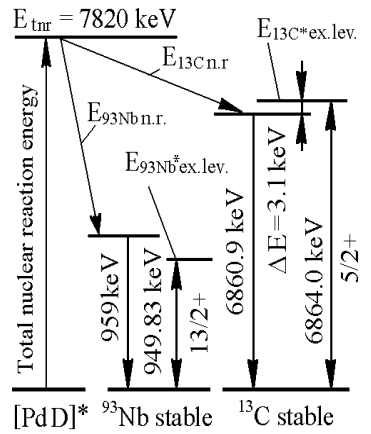

a

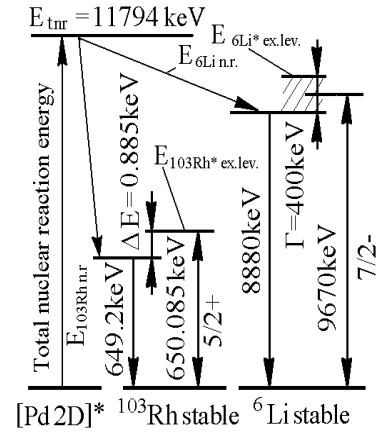

b
Fig. 20 Schematic of proposed long-ranged (resonant) nuclear reactions; (a) for $\mathrm{Pd}+\mathrm{D}$ transmutation reaction; (b) for Pd + 2D transmutation reaction.

product nucleus 13C should receive $6.8609 \mathrm{MeV}$, and that the product nucleus ${ }^{93} \mathrm{Nb}$ should receive $0.959 \mathrm{MeV}$ (in connection with the reaction scheme of Fig. 20a). Note that a nuclear excited state (nuclear isomer) with an energy of $6.864 \mathrm{MeV}$ (and excited level width of 6 $\mathrm{keV}$ ) exists for ${ }^{13} \mathrm{C}$, and that an excited level with an energy of $0.94983 \mathrm{MeV}$ exists for $\mathrm{Nb}$.

The difference between the energy received by nuclide ${ }^{13} \mathrm{C}$ and the energy of the excited level is 3.1 $\mathrm{keV}$. If the crystalline lattice has available $1.5 \mathrm{keV}$, and given the $6 \mathrm{keV}$ width of the excited energy level, one might expect that there should be a high probability for such a long-range (resonant) nuclear reaction to occur.

Consider the reaction (Fig. 20b):

${ }^{105} \mathrm{Pd}+2 \mathrm{D} \rightarrow{ }^{109}[\mathrm{Pd} 2 \mathrm{D}] \rightarrow{ }^{93} \mathrm{Rh}+{ }^{6} \mathrm{Li}+11.794 \mathrm{MeV}$

Energy and momentum conservation again dictate that the product $6 \mathrm{Li}$ nucleus should end up with an energy of $8.880 \mathrm{MeV}$. A nearby excited state is available, so a similar situation can occur as indicated in Fig. 20b.

The totality of the experimental results allows us to assume that the energy of the excited nuclear levels of the product nuclei is converted into heat. The specific physical mechanism of such conversion will require additional research.

\section{Conclusions}

We have reviewed many results that we have obtained in glow discharge experiments and in 
high-voltage electrolysis experiments. We have observed excess power in glow discharge experiments up to $10 \mathrm{~W} / \mathrm{cm}^{2}$, and with a power gain of up to $170 \%$; in the high-voltage electrolysis experiments we have seen excess power up to $300 \mathrm{~W}$, with a power gain as high as $340 \%$. These experiments clearly demonstrate the presence of an interesting new source of thermal power and energy. We have reviewed results obtained in our search for nuclear products, where many new elements and isotopes appear to have been produced. We have observed both collimated and diffuse X-ray emission in the $\mathrm{keV}$ regime, which constitutes a new fundamental effect that we discovered. Finally, we have presented a discussion of our results in terms of conjectures and hypotheses concerning physical mechanisms and reactions that may be involved (but which will require further study in order to determine whether they are correct or not).

\section{Acknowledgments}

The authors are happy to express our thanks to: Runar Kuzmin professor, solid and nuclear physic, Moscow State University, Sergey Pikuz, Doctor Ph. D., plasma physic, Russian Academy Lebedev Physics Institute, George H. Miley, professor, plasma and nuclear physic, Ill. University, Peter L. Hagelstein, professor, plasma and laser physic, MIT, for their help in this work.

\section{References}

[1] A.B. Karabut, Y.R. Kucherov, I.B. Savvatimova, Nuclear product ratio for glow discharge in deuterium, Phys. Lett. A. 170 (1992) 265-272.
[2] A.B. Karabut, Experimental registration of a high current glow discharge of the exited long living atomic levels with the energy of 1-3 keV and nuclear products emission in the solid medium, in: Proc. 11 Int. Conf. Emerging Nuclear Energy Systems (ICENES 2002), Albuquerque, New Mexico, USA, 2002, pp. 408-416.

[3] A.B. Karabut, E.A. Karabut, The electric and heat measurements in high voltage electric discharge system experiments, in: Proceedings of the 14th International Conference on Cold Fusion, 2008, USA, pp. 169-175.

[4] A.B. Karabut, Analysis of experimental results on excess heat power production, impurity nuclides yield in the cathode material and penetrating radiation in experiments with high current glow discharge, in: Proc. 8th Int. Conf. Cold Fusion, Italy, 2000, pp. 329-334.

[5] A.G. Lipson, A.S. Roussetski, A.B. Karabut, G.H. Miley, Gain of dd-reaction and X-ray emission generation in high current pulsed glow discharge in deuterium with Ti-cathode at $0.8-2.45 \mathrm{kV}$ voltage, Journal of Experimental and Theoretical Physics $100 \quad$ (2005) 1175-1189. (in Russia)

[6] A.B. Karabut, Production of excess heat, impurity elements and unnatural isotopic ratios formed at excited long-lived atomic levels with energy of more than $1 \mathrm{keV}$ in a solid cathode medium during high-current glow discharge, in: Proc. 10th Int. Conf. On Cold Fusion, Cambridge, MA, USA, 2003, pp. 99-111.

[7] A.B. Karabut, Study of deuterium loading into Pd cathode samples of glow discharge, in: Proc. 9th Int. Workshop on Anomalies in Hydrogen/Deuterium Gas Loaded Metals, Siena, Italy, 2010.

[8] A.B. Karabut, Research into low energy nuclear reaction in cathode sample solid with production of excess heat, stable and radioactive impurity nuclides, in: Proc. 12th Int. Conf. Cold Fusion, Japan, 2005, pp. 214-230.

[9] A.B. Karabut, Experimental results on excess power heat, impurity nuclides, and X-ray production in experiments with a high-voltage electric discharge system, Journal Condensed Matter Nucl. Sci. 6 (2011) $1-19$. 


\title{
Proposition of a New Type of Jointless System For Existing Concrete Bridges
}

\author{
Yuichi Ishikawa ${ }^{1}$, Minobu Aoyama ${ }^{1}$, Masami Kuroyanagi ${ }^{2}$, Masatugu Nagai ${ }^{3}$ and Takeshi Miyashita ${ }^{3}$ \\ 1. Kanazawa Branch, Central Nippon Highway Engineering Nagoya Co. Ltd., Kanazawa 920-0025, Japan \\ 2. Nagoya Branch, Central Nippon Expressway Co. Ltd., Nagoya 460-0003, Japan \\ 3. Department of Civil and Environmental Engineering, Nagaoka University of Technology, Nagaoka 940-2188, Japan
}

Received: August 06, 2013 /Accepted: October, 21, 2013 /Published: February 15, 2014.

\begin{abstract}
Since 1990s, the use of deicing salts (i.e., chlorides) has dramatically increased in areas with heavy snowfall in Japan. As a result, the water mixed with salts has accelerated the damage of the reinforced concrete (hereafter, RC) structures. Recently conducted inspection results of RC bridges have reported that many of the damages or deteriorations are observed at the girder ends and abutments. This is caused from the water leakage due to the aged expansion joints. In general, the cost for repairing the damaged RC structures is much higher than that for renewing the expansion joints. Therefore, to prevent these damages, we developed a new highly durable jointless system, named RC plug joint, for existing RC bridges with a bridge length less than 40 meters. The RC plug joint connects the abutment's backwall to the superstructure's deck using reinforcing steel bars and fiber reinforced concrete. The newly developed RC plug joint system can prevent water leakage and allow for a smooth ride of vehicles at the joint. This paper will explore and discuss the development of the RC plug joint, analysis of conducted investigations, and future installation methods.
\end{abstract}

Key words: Jointless system, RC plug joint, field investigation, leakage assessment, travelling performance.

\section{Introduction}

The use of deicing salts dramatically increased in heavy snowfall areas in Japan as a result of studded tired being banned in 1990 [1]. Fig. 1 shows the volume of deicing salts used on the Hokuriku Expressway. The volume of deicing salts used has steadily increased. The aged bridge expansion joints with the deteriorated waterproof function allow the remnants of dissolved deicing salts, rainwater and melted snow to drop down to the girder. As a result, many reinforced concrete (hereafter, RC) girders, abutments and piers have been damaged by salt attack. Figs. 2 and 3 show the typical damage of an RC girders end and abutments due to leakage from aged expansion joints. The delamination of concrete and exposure of reinforcing bars (hereafter, rebars) are found in the locally

Corresponding authors: Yuichi Ishikawa, M. Eng., research field: maintenance of bridges. E-mail: y.ishikawa.a@c-nexco-hen.jp. deteriorated concrete girders and abutments. Therefore, we developed a new highly durable jointless system, named RC plug joint, for existing RC bridges with a bridge length less than $40 \mathrm{~m}$. The RC plug joint connects the abutment's backwall to the superstructure's deck using reinforcing steel bars and fiber reinforced concrete. This paper will explore and discuss the development process, structural detail, installation method and conducted investigations.

\section{Investigations of the Aged Expansion Joints}

\subsection{Investigation of Water Leakage}

Fig. 4 shows the results of the investigation of water leakage from several types of the expansion joints in the Hokuriku Expressway. Water leakage was reported in all kinds of existing expansion joints. It was also reported that salt attack occurred in RC structures around the aged joints [2]. The sealed comb joint was 


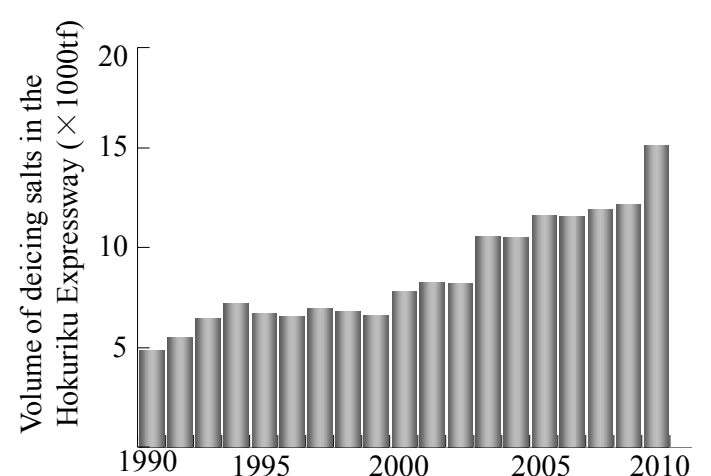

Fig. 1 The volume of deicing salts in the Hokuriku Expressway.

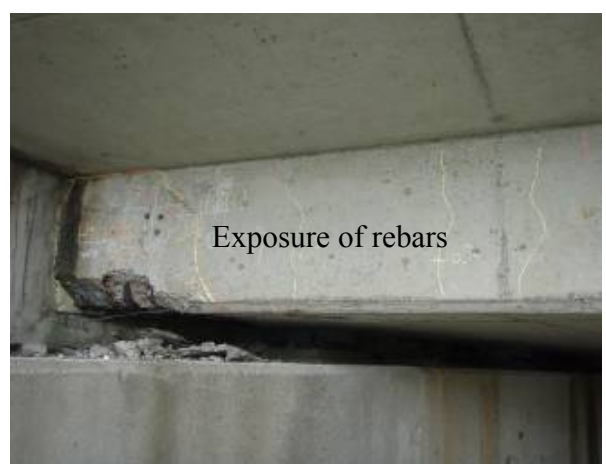

Fig. 2 Typical damage of a concrete bridge girder end.

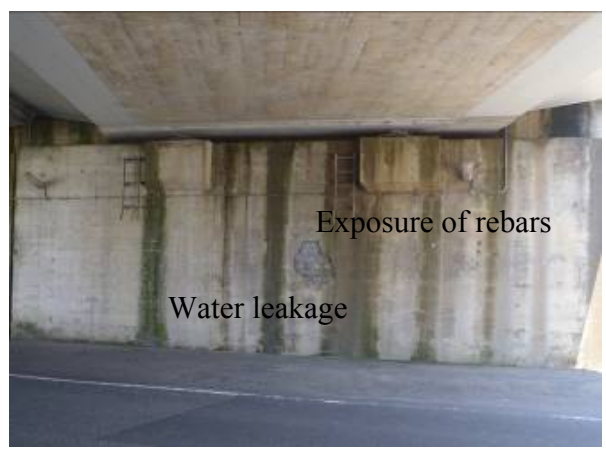

Fig. 3 Typical damage of an bridge abutment.

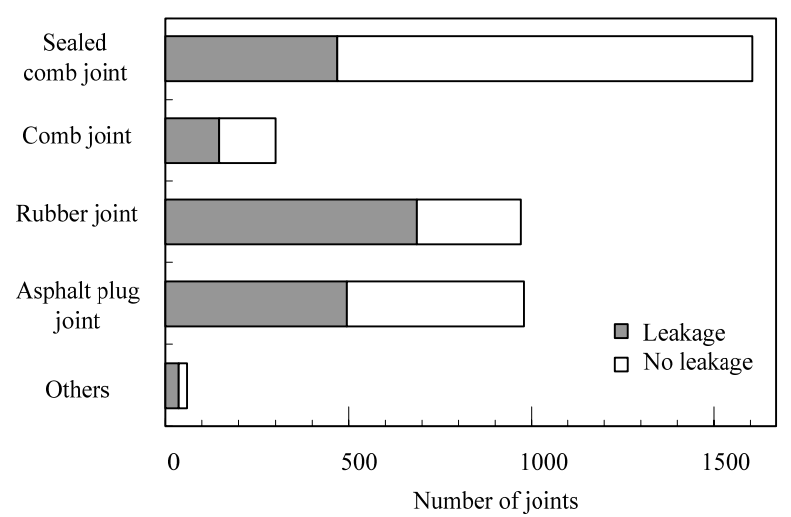

Fig. 4 Investigation of water leakage from several types of expansion joints [2]. expected to be the least leaking type among the conventional expansion joints. However, its leakage reached around $30 \%$. Much of the leakage was caused by accumulated dust and sand within the drainage. The accumulated dust and sand in the drainage created a narrow path allowing rainwater to overflow. In addition, the deicing salts corroded the drainage channel, thus increasing the leakage. Fig. 5 shows water leakage from aged expansion joints.

In the past, we worked to replace or improve the aged expansion joints, but it proved too difficult to prevent the water leakage completely.

\subsection{Typical Damage of Expansion Joints}

As a result of heavy traffic, the bridge expansion joints were found to be the most damaged part in the bridge elements. In addition, the heavy cyclic traffic loads caused poor conditions around the expansion joints.

Fig. 6 shows a typical damaged area around the aged expansion joints. The remnants of dissolved deicing salts, rainwater and melted snow eventually saturate the joints and leakage begins.

Fig. 7 shows the accumulated amount of the local damages and repairs due to the leakage from the aged expansion joints. The locally deteriorated areas were inspected between $2 \mathrm{~m}$ from the edge of RC bridge girders and abutments. The evaluation of the inspection data shows the number of the locally deteriorated areas

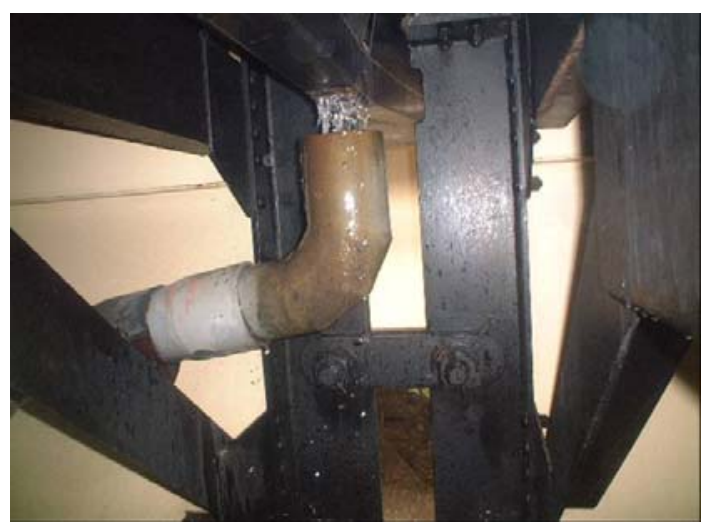

Fig. 5 Water leakage from the sealed comb joint. This is one of the most serious problems on managing expressway bridges. 


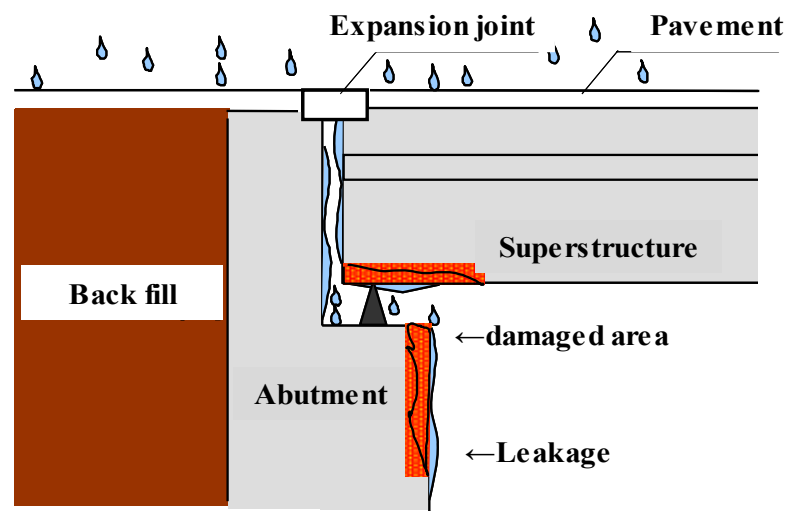

Fig. 6 Leakage from an aged expansion joint and damaged concrete area was occurred.

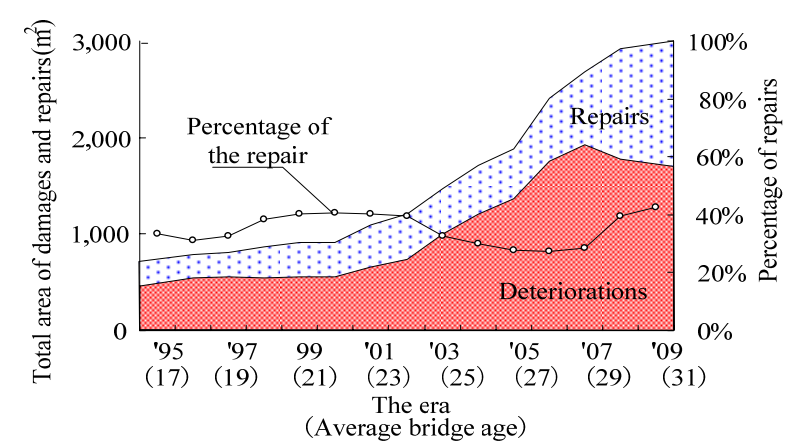

Fig. 7 Results of the inspection of bridges in the Hokuriku Expressway.

has dramatically increased since 2000. Currently, the percentage of the repairs (i.e., total repaired area divided by total deteriorated area) is around $40 \%$, forcing the continuation of the repair work inevitable. In general, the repairing cost of structural damages by salt attack is much higher than the cost for renewing the expansion joints [3].

\section{Outline of Reinforced Concrete Plug Joint}

\subsection{Basic Concepts}

Fig. 8 shows the side view of the RC plug joint. In this system, the aged expansion joint is removed completely, and fiber reinforced concrete fills the space. Then, a waterproof membrane is applied on the surface of the fiber reinforced concrete. Lastly, a new asphalt pavement layer is completed.

\subsection{Structural Characteristics}

The RC plug joint connects the abutment's backwall (i.e., parapet) to the superstructure's deck using fiber reinforced concrete. This jointless system is applicable to existing concrete bridges whose length is less than 40 meters. Due to temperature change, deformation occurs as shown in Fig. 9. However, it was confirmed that the strain in the rebars was low level. As a result, the prevention of leakage in addition to creating an easier passage for vehicles at the expansion joint is achieved. Therefore, the superiority of this system compared to the previously used expansion joint devices is quite clear.

\subsection{Details of RC Plug Joint}

A detailed view of an RC plug joint is shown in Fig. 10. The RC plug joint completes the gap between the girder and abutment with fiber reinforced concrete for the main purpose of preventing water leakage from the expansion joint. In addition, the deck waterproofing and the road asphalt pavement are applied on top of the RC plug joint. This double protection system guarantees increased waterproof protection in comparison with the previously used type of expansion

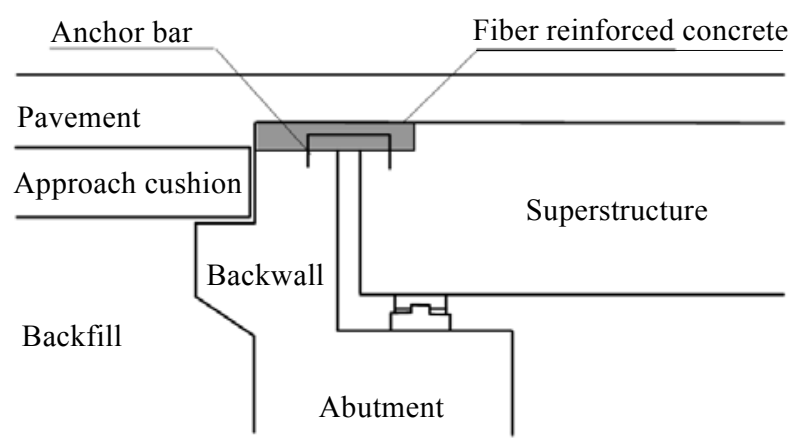

Fig. 8 Side view of the RC plug joint.

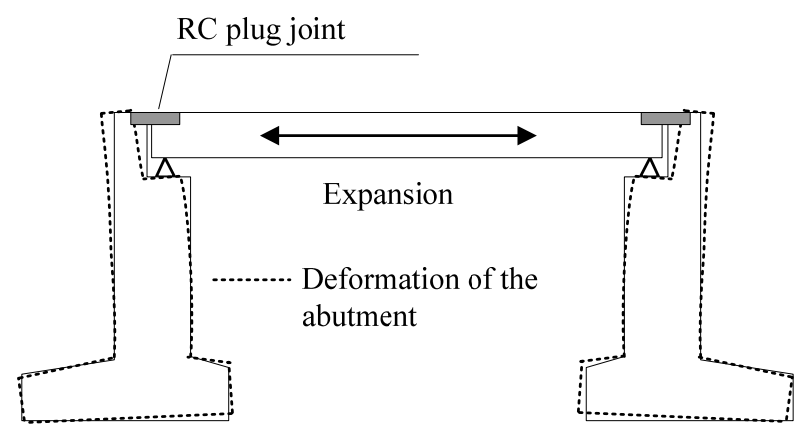

Fig. 9 Deformation of the abutment and foundation. 


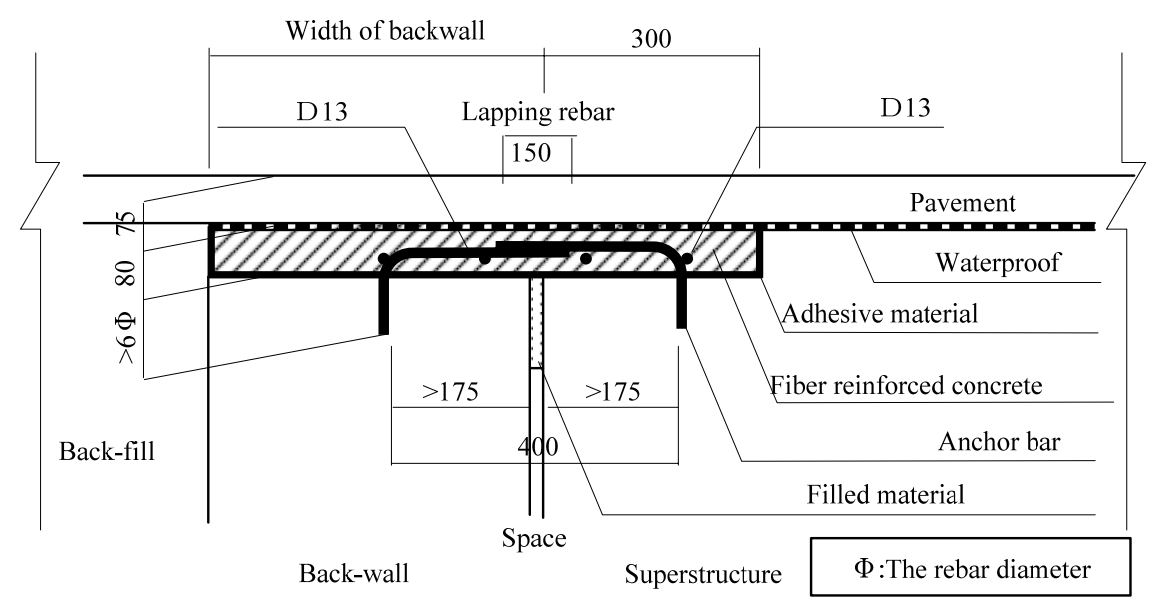

Fig. 10 Details of the RC plug joint (Unit: $\mathbf{m m}$ ).

joint system. A concrete adhesive material is applied to the interface of the concrete to ensure bonding and cohesion of both the old and new concrete with the steel anchor bars. Furthermore, it is predicted that the $\mathrm{RC}$ plug joint could possibly collapse in the event of a large-scale earthquake because the length of embedding rebar is 6 times the rebar diameter $\varphi$.

\subsection{Installation of RC Plug Joint}

The installation of the RC plug joint is executed in phases, to avoid traffic interruption. The repairs are usually conducted in daytime.

Table 1 shows the mix proportion of fiber reinforced concrete. The repairing concrete has short cure time and compressive strength reach to $24 \mathrm{~N} / \mathrm{mm}^{2}$ within only $3 \mathrm{~h}$. Moreover, the tensile strength of fiber reinforced concrete is stronger than conventional concrete.

In many cases, the replacement of old expansion joints in expressway bridges has weakened the existing concrete surface and corroded the rebars of these structures. In addition, the durability of RC plug joints is greatly affected by the adhesion strength to the existing concrete. For this reason, we carefully removed the existing aged joints, the concrete delaminated areas and the remaining loose materials. We also conducted a visual inspection and hammer tapping test to assess the soundness of the existing concrete surface. Then we coated the concrete surface with the adhesion material and poured concrete. Fig. 11 shows the installation of the RC plug joint. All processes were completed within $8 \mathrm{~h}$. During the following days, we set a waterproofing membrane on the concrete surface and paved the asphalt layer.

\section{Results of Field Investigation}

\subsection{Leakage Assessment}

As of 2012, we have replaced the existing joints with the RC plug joints at 85 locations within the area under supervision of Kanazawa Branch, Central Nippon Expressway Co., Ltd. Fig. 12 shows the number of installed RC plug joints and results of leakage assessment. Unfortunately, the water leakage from the RC plug joints was found at three locations. The faults of RC plug joints occurred due to the debonding of the

Table 1 Mix proportions of fiber reinforced concrete.

\begin{tabular}{lllllllll}
\hline \multirow{2}{*}{ Fiber material } & \multirow{2}{*}{$\mathrm{W} / \mathrm{C}(\%)$} & $\mathrm{s} / \mathrm{a}(\%)$ & $\mathrm{T}$ & $\mathrm{C}$ & $\mathrm{S}$ & $\mathrm{G}$ & Fiber & Admixture \\
\cline { 5 - 9 } & & & $\mathrm{W} \times \%)$ & 2.0 \\
\hline Polypropylene fiber & 39.7 & 51.9 & 174 & 438 & 864 & 807 & 11.6 & 2.0 \\
Vinylon fiber & 40.3 & 52.6 & 184 & 457 & 853 & 775 & 16.5 & 2.0 \\
\hline
\end{tabular}




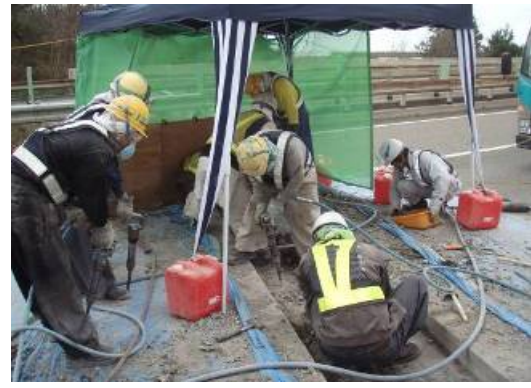

(a)

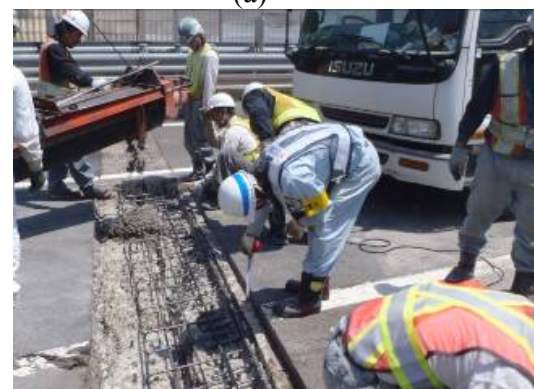

(d)

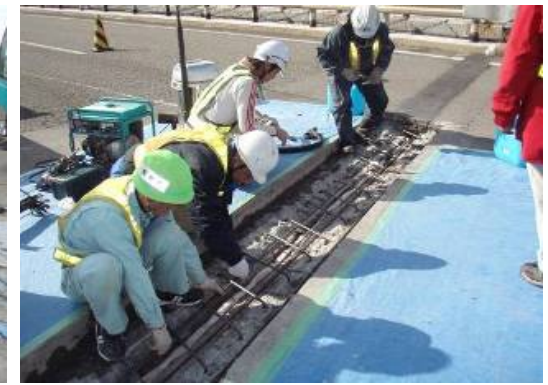

(b)

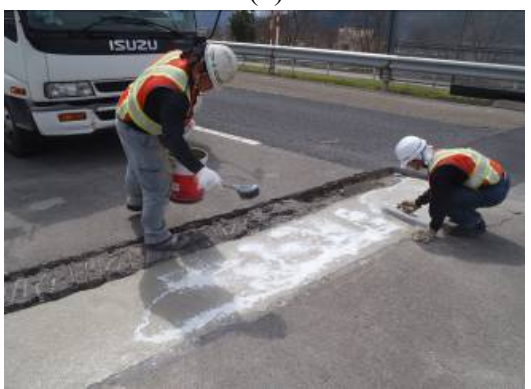

(e)

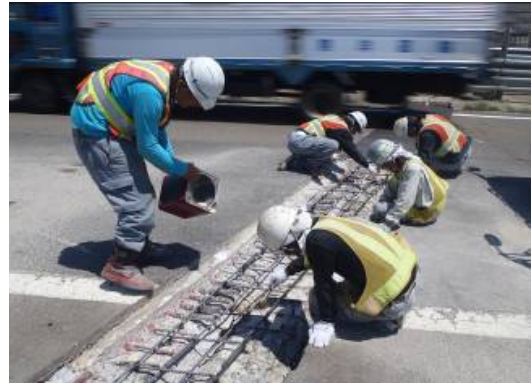

(c)

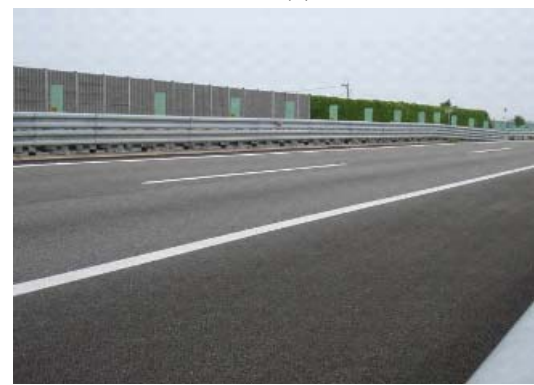

(f)

Fig. 11 Installation of the RC plug joint: (a) removing the aged joint; (b) setting rebars; (c) painting with the adhesion material; (d) pouring concrete; (e) curing the concrete and (f) paving the asphalt layer.

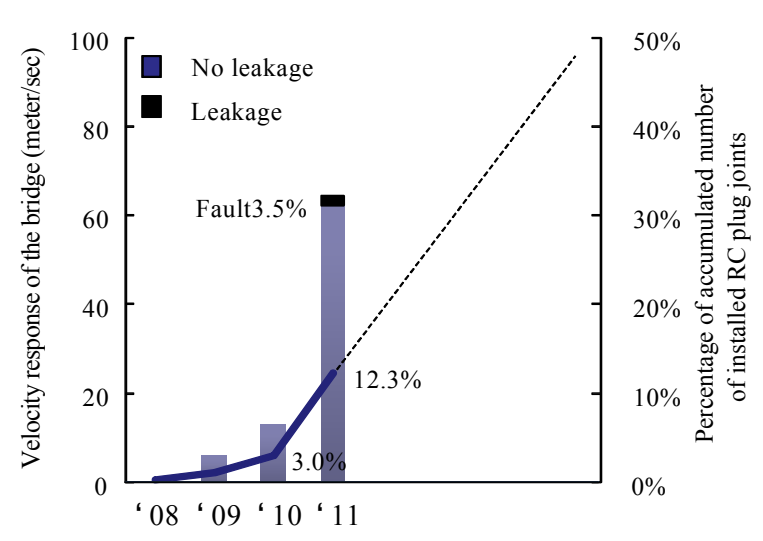

Fig. 12 Leakage assessment of RC plug joints.

existing surface concrete. After much discussion over this fault, we decided to use an adhesion material (i.e., epoxy resin) on the existing surface concrete for future repairs.

There has been no subsequent pavement damage such as potholes, cracks or leakage from the RC plug joints. From these findings, it is confirmed that the reliability of the $\mathrm{RC}$ plug joint proves thus far successful and we intend to continue monitoring their performance.

\subsection{Travelling Performance}

In order to assess the effect of vibration reduction due to traffic loading after the installation of an RC plug joint, the vibration response of both, the bridge and test car was measured. Fig. 13 shows the reduction effect of the bridge response before and after the installation of the RC plug joint. These results show that the $\mathrm{RC}$ plug joint has a great reducing effect on the bridge's vibration. After comparing the results before and after the RC plug joint was installed, it is observed that a smoother passage for vehicles was also achieved.

\subsection{Measurement of Strain of Anchor Bars}

Fig. 14 shows the measured strain of anchor bars under traffic loads. Fig. 15 shows the strain of anchor bars due to seasonal temperature variation. It was concluded that the strain due to temperature variation was 50 times higher than that caused by the traffic loading. As a result of this discrepancy, future attention must be paid to the temperature variation during the design phase. Furthermore, a structural analysis on the effect of temperature variation using a frame model concluded that the permissible expansion and contraction of the RC plug joint should be limited to $20 \mathrm{~mm}$ for bridge lengths less than $40 \mathrm{~m}$. 


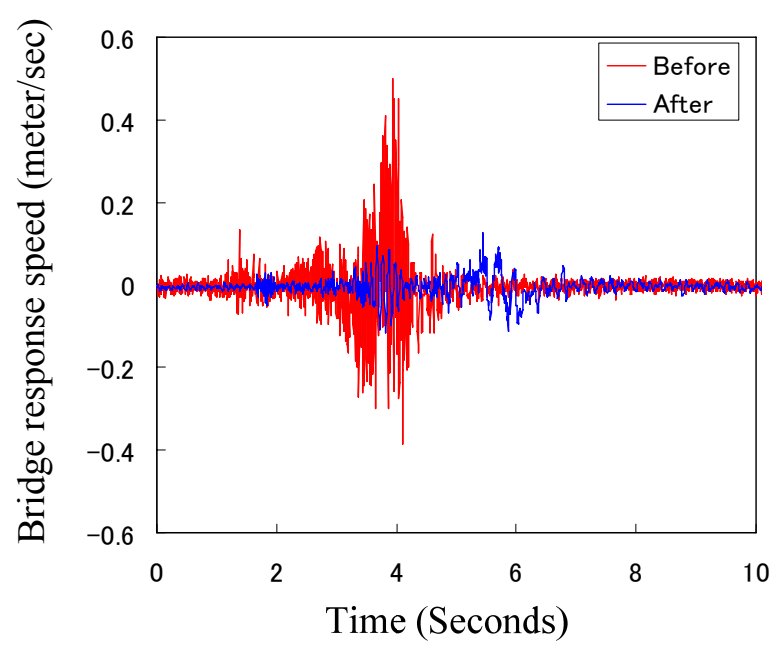

Fig. 13 Velocity response of the bridge caused by test car.

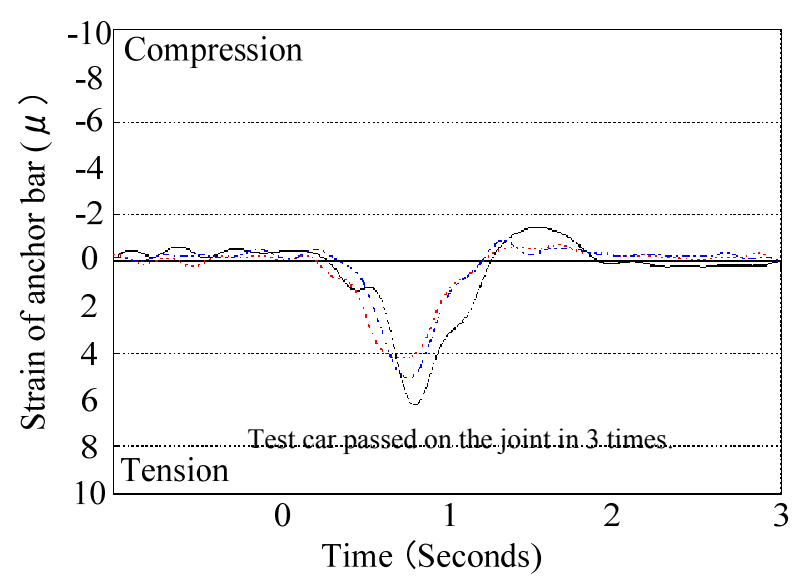

Fig. 14 Measured strain induced by test cars.

\section{Conclusions}

This paper discusses the development of the RC plug joint system as well as analyzes the results of the investigations conducted. This particular method of

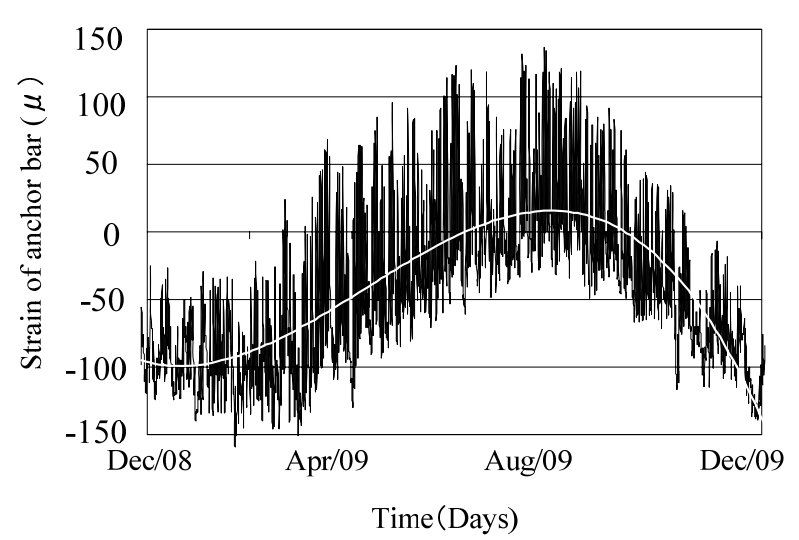

Fig. 15 Measured strain due to temperature variation.

installation is expected to be the most cost-effective one. In the next phase of development, we propose to work on a simple design and establish a standard of installation, while conducting further replacement of existing deteriorated expansion joints. Although the safety, serviceability and durability of the RC plug joints have been confirmed, the authors intend to conduct further field surveys and monitor the long-term performance of the RC plug joints.

\section{References}

[1] JCI, Technical Reports of Damaged Reinforced Concrete Structures Due to Deicing salts, 1999. (in Japanese)

[2] B. Yanev, Bridge Management, John Wiley \& Sons. Inc., 2007.

[3] K. Kumagai, H.T akahashi, M. Aoyama, The damage characteristic and degradation process of the concrete components of the bridge beam end in Hokuriku area, Journal of Japan Society of Civil Engineers 68 (798) (2005) 31-39. (in Japanese) 


\title{
The Parameters of the Stellarator as a Neutron Source for a Subcritical Reactor
}

\author{
Vasiliy Rudakov \\ Institute of Plasma Physics National Science Center “Kharkov Institute of Physics and Technology”, Kharkov 61108, Ukraine
}

Received: July 15, 2013 / Accepted: August 06, 2013 / Published: February 15, 2014.

\begin{abstract}
The possibility of developing a stellarator-based neutron source designed for the nuclear reaction initiation in the blanket of hybrid reactor is studied. An analog of the Large Helical Device (LHD) stellarator design, with linear dimensions increased by a factor of 1.5 is taken for the magnetic system. Plasma parameters and the deuterium-tritium (DT) mixture fusion power are calculated using the space-time numerical code under the assumption of the neoclassical transport in the ambipolarity regime. Using the $10 \mathrm{MW}$ plasma heating sources, it is possible to obtain the DT fusion power of one-to-two tens MW.
\end{abstract}

Key words: Stellarator, subcritical reactor, ambipolar electric field, neoclassical transport, neutron source, fusion power.

\section{Introduction}

Investigations into the controlled thermonuclear fusion are going on during more than fifty years, but there is no last answer to the problem. Putting into operation of an experimental fusion reactor ITER, planned by the end of the current decade, will be only the next, though important, step on the way of problem solving.

At the same time, there are existing long-discussed alternative paths for development of power reactors using the nuclear fusion as a neutron source for power multiplying by the nuclear fission of heavy elements or for the accumulation of a fuel to be used in nuclear reactors. In the first case for energy multiplying one can use uranium enriched by isotope ${ }^{235} U$ having the multiplication factor less than unity. In another case the fusion-produced neutrons participate in nuclear transformations of nonfissionable heavy elements into elements which can be used as a fuel for nuclear power plants. For this purpose, it is possible to use

Corresponding author: Vasiliy Rudakov, Ph.D., senior research worker, research fields: thermonuclear fusion, reactor-stellarator problems, plasma confinement and magnetic field calcullations. E-mail: rudakov@kipt.kharkov.ua. uranium-238 and thorium-232. These elements absorb neutrons and after subsequent decays are transformed into plutonium-239 and uranium-233 respectively.

In one DT-fusion event the energy of $17.5 \mathrm{MeV}$ is released with $\alpha$-particle and neutron production, and one thermonuclear neutron fission of a heavy element gives near $200 \mathrm{MeV}$. And neutrons, produced as a result of nuclear fission, can evoke new fissions. A number of such fissions is determined by the multiplication factor $K_{m}$, which at $K_{m}<1$, according to the Ref. [1], equals to $\eta=1 /\left(1-K_{m}\right) / v_{f}$, where, $v_{f}$ is a number of neutrons produced by one fission event. In the case of $K_{m}>1$ the system comes into the uncontrolled chain reaction regime. Thus, when, for example, the multiplication system provides the number of fission events per one external neutron $\eta=$ 10 , the reactor power can be increased more than by a factor of 100 as compared with the power of a thermonuclear neutron source. Consequently, to develop a Giga-Watt-power reactor, it is sufficient to have a thermonuclear device with a fusion power of $10 \mathrm{MW}$.

Unlike a traditional reactor based on the nuclear fission, where the multiplication factor is equal to unit, 
in the reaction under consideration the probability of uncontrolled chain reaction appearance at $K_{m}<1$ takes no place.

Combination of fusion reactions and nuclear fission in one and the same device has determined the name of such systems, as hybrid reactors. Fusion-fission hybrid reactors have been considered as power sources as early as investigations into the problem of controlled thermonuclear fusion commenced. A detailed analysis of such reactors, developed on the base of thermonuclear traps -tokamaks, has been carried out in the USSR by Golovin and Orlov [2, 3]. A design of a Hybrid Thermonuclear Tokamak Reactor (HTTR) has been proposed Velikhov [4]. On the initiative by I.N. Golovin the Kharkov researchers started designing of an experimental fusion facility TB-0 [5]. Unfortunately, the project was not realized.

An accident at the Chernobyl nuclear power plant has reduced for a certain time the interest in hybrid systems and livened up the works in the direction of developing clean fusion reactors, in particular, the International Tokamak Exsperimental Reactor (ITER) project. However, to solve the energy problems by means of clean fusion reactors is a long-term outlook, therefore, during the recent years the interest in developing hybrid systems revived [6-9]. Feasibility of these designs become real as at modern thermonuclear facilities a nuclear fusion power of $10 \mathrm{MW}$ has been reached $[10,11]$.

Up to date fusion-fission hybrid reactor designs were developed, in general, on the base of tokamaks. A tokamak's disadvantage is that to maintain the stationary fusion reaction in the system is impossible. Therefore, there are some technical difficulties for the development of a stationary power source on the base of tokamaks. There is no such a problem in a stellarator, where to maintain the fusion reaction is possible. At the same time, experimental studies on the fusion plasma in stellarators have not yet reached the level of research at tokamaks, though the features of plasma maintenance observed in experiments obey nearly the same laws as these noticed in tokamaks [12].

Theoretical investigations into the plasma loss mechanisms in the stellarator systems resulted in the development of a neoclassical transport theory [13-15], permitting to take into account an ambipolar electric filed leading to the flattening between the ion and electron plasma fluxes. In Refs. [16-18], a comparison is done for experimentally measured electric field profiles in the Wendenstein VII-AS stellarator (W7-AS) and these calculated by the neoclassical theory. In the calculations of $E_{r}$ the plasma density and temperature profiles measured experimentally were used. A satisfactory accordance between the theory and experiment is obtained.

In the paper by Wakasa et al. [19], experimental results obtained in three stellarators (LHD, W7-AS and Spain stellarator TJ-II) are compared with neoclassical theory data. The electric field values were calculated using the Drift Kinetic Equation Solver (DKES) code. A good agreement between the calculated and experimental results is shown for LHD and TJ-II facilities, at least for the major plasma part. A certain correspondence between the experiment and neoclassical theory for the central region of the plasma column was obtained for the LHD and TJ-II facilities by Olaya [20].

Results of the comparison between the plasma confinement theory and experiments in stellarators give grounds for forecasting the expected plasma parameters in experimental facilities of the next generation, in particular, in the systems that might be used for the development of neutron sources. The transport coefficients in the neoclassical theory are the functions of local plasma parameters that enable to obtain their spatial distributions by solving the set of equations such as diffusion equations and heat conduction equations. Similar solutions were obtained in the calculations of plasma parameter for different variants of the stellarator-reactor: experimental [21], demonstration [22] and power [23] reactors. It has been shown that under certain assumptions on the processes 
of fuel loading and plasma heating, the stellarator-reactor, with comparable dimensions, can have energy characteristics like to these of corresponding-type tokamak-reactors.

The present work is aimed to the study of a possibility of developing the stellarator -based neutron source designed for the initiation of nuclear reactions in the hybrid reactor blanket. An analog of the LHD stellarator design was taken as a magnetic system with linear dimensions increased by a factor of 1.5. Thus the stellarator parameters become comparable with the parameters of existing experimental facilities of a tokamak type. In addition, the sizes of the system provide enough space to accommodate the breeding blanket around the plasma.

The plasma parameters and the DT mixture fusion power were calculated by the space-time numerical code earlier used for the reactor parameter calculations in Refs. [21-23].

\section{Set of Equation and Numerical Model}

The set of equations solved in Refs. [22, 23] describes the space-time behavior of the plasma in the stellarator reactor. The system assumes a one-dimensional spatial dependence of plasma parameters on the averaged minor plasma radius.

$$
\begin{gathered}
\frac{3}{2} N \frac{\partial T_{\mathrm{e}}}{\partial t}=-\frac{1}{r} \frac{\partial}{\partial r} r \Pi_{\mathrm{e}}+\frac{K_{f} N^{2}\langle\sigma v\rangle}{4} E_{\alpha}+ \\
Q_{h e}-Q_{e i}-Q_{b}-Q_{c}+Q_{E} \\
\frac{3}{2} N \frac{\partial T_{i}}{\partial t}=-\frac{1}{r} \frac{\partial}{\partial r} r \Pi_{i}+Q_{e i}+Q_{h i}-Q_{E} \\
\frac{\partial N}{\partial t}=-\frac{1}{r} \frac{\partial}{\partial r} r S_{j}+S_{\delta}
\end{gathered}
$$

The first two equations describe the space-time behavior of the electron Eq. (1) and the ion Eq. (2) temperature. The third one is a plasma particle diffusion equation. The first term on the right side of equation for the electron heat conduction Eq. (1) determines the temperature variation as a result of the heat flux corresponding to the neoclassical theory Kovrizhnykh [14]. The second term on the right describes the heating of electrons produced in the process of fusion by $3.52 \mathrm{MeV} \alpha$-particles. An equal content of deuterium and tritium in the reactor plasma is assumed. The coefficient $K_{f}$ determines the part of $\alpha$-particle energy given to electrons. It is supposed that $95 \%$ of $\alpha$-particle energy is absorbed by the plasma. The rest terms on the right sides of Eqs. (1) and (2) denote respectively: $Q_{h i}$ and $Q_{h e}$ are the heating of ions and electrons from the external sources, $Q_{e i}$-electron-ion heat exchange as a result of Coulomb collisions, $Q_{b}$-bremsstrahlung, $Q_{c}$-cyclotron radiation, $Q_{E}$-energy change of particles in the ambipolar electric field during their radial motion. The cyclotron radiation is well-reflected from the chamber wall, therefore in the calculations the reflection coefficient for this radiation is taken equal to 0.95 . Consequently, only $5 \%$ of the power is absorbed by the reactor walls.

Calculations were carried out with the use of different models of plasma heating by the external sources: separated heating of electrons and ions or simultaneous heating of both components. Also, it has been assumed that the specific heating power is proportional to the plasma density: $Q_{h j}=q_{h j} N$, where j denotes electrons or ions according to the equation. The total heating power was determined by the integral over the plasma volume:

$$
\mathrm{P}_{\mathrm{hj}}=\oint_{V} Q_{h j} d v
$$

The specific energy release due to the effect of the ambipolar electric field has been taken by the value $Q_{\mathrm{E}}=-S_{j} E_{r}$ under conditions when the diffusion fluxes of electron and ions are equal: $S_{\mathrm{e}}=S_{\mathrm{i}}$.

Here, as in the Ref. [23], the heat fluxes and diffusion particle fluxes determined in the Ref. [14] are used. It has been assumed that the plasma electrons are in the regime when the diffusion coefficient has an inverse relationship with the collision frequency $v$, and the ion diffusion obeys to the relationship $\sqrt{v}$. 
The density equation Eq. (3) contains on the right side, besides a diffusion term, a term with the particle source $S_{\delta}$, used for the simulation of the plasma density maintenance at an approximately constant level. Diffusion plasma losses were compensated by the model of the fuel pellet injection. It has been shown earlier [23] that the fuel injection into the central plasma column region is optimum one from the point of view of plasma confinement.

The set of Eqs. (1)-(3) was enlarged by boundary and initial conditions. Besides, the spatial derivatives of density, temperature and potential were assumed to be zero in the plasma column center:

$$
T_{j, x=0}=0, N_{x=0}^{\prime}=0, \Phi_{x=0}=0,
$$

and the initial conditions satisfy the expressions:

$$
T_{j, t=0}=T_{j, 0}\left(1-x^{l}\right)+\delta_{T}, N_{j, t=0}=N_{0}\left(1-x^{l}\right)+\delta_{n}
$$

where $j$ denotes the particle sort, $x$ is the current radius normalized to unit. The constants $\delta_{\mathrm{n}}$ and $\delta_{\mathrm{T}}$ determine the values of the plasma density and temperature at the plasma column edge. The index $l$, being an integer, in the most of calculations was assumed as $l=2$.

The ambipolar electric field value was determined from the equation for the equality between the ion and electron fluxes $S_{e}=S_{i}$ at each step of the numerical code space network. In the present paper the electric field in every case was determined by the left (ion) root of the equation for the equality of fluxes giving its negative values. Such a choice is not optimum from the point of view of reactor confinement properties. A regime in which positive values of the electric field (electron root) are realized [14] might be more suitable. The electron root realization occurs, as a rule, with lower values of fluxes $S_{j}$. However, the solutions with the right root do not exist in every point of the plasma radius, while the negative root is always presented.

\section{Calculation Results}

In the neoclassical theory the heat and diffusion fluxes of plasma electrons depend on the effective helical ripple $\varepsilon_{h}$. Its value in different experimental facilities is varying within the wide ranges, and even under conditions of a single facility the value of $\varepsilon_{h}$ undergoes appreciable changes when the vertical magnetic field and the plasma pressure $\beta$ are varying [24]. In this paper, as in the Ref. [23] for simulation of the amplitude of magnetic field helical disturbances the following expression is used:

$$
\varepsilon_{h}=\varepsilon_{h 0}+0,05 r^{0,87}
$$

where, $\varepsilon_{h 0}=0.01$, that gives at the plasma edge $\varepsilon_{\text {max }}=0.06 \mathrm{n}$. In the LHD stellarator with magnetic axis shifted by the radius $R=3.53 \mathrm{~m}$, the effective ripple at the plasma edge is equal to 0.05 [25].

Plasma density maintenance at an approximately constant level was simulated by the fuel pellet injection under the assumption of the triangular model ablation $\delta n=n_{p}(1-|\alpha 2-\chi| / \Delta)$, where $a_{2}$ is the radius of injection center and $\Delta$ is the half-width of injection region. The pellet size was equal to $1 \%$ of the total number of particles in the reactor plasma. When the complete particle number decreases below 0.99 the next pellet is injected. The energy loss by heating the injected particles is taken into account via the condition of thermal plasma energy conservation. The energy loss by pellet evaporation was not taken into account because of its small quantity.

As for geometric parameters, an analog of the LHD stellarator design, with linear dimensions increased by a factor of 1.5, was taken with the minor plasma radius of one meter $(a=1 \mathrm{~m})$ and the major tore radius $R=6 \mathrm{~m}$. In all the calculations a basic magnetic field was taken equal to $5 \mathrm{~T}$.

The problem was reduced to the calculation of the space-time behavior of plasma parameters with the given levels of the plasma heating power and in the regime of fuel pellet injection. In the most of calculations a $10 \mathrm{MW}$ power from the external source was loaded into the plasma. In a different of calculations the power was loaded wholly into ions or separately into electrons. Also, there were calculations 
on the simultaneous heating of ions and electrons with equal parts of contribution.

It has been shown in Ref. [21] that the maximum plasma parameters, from the point of view of heating and confinement, are reached in the case of fuel injection into the plasma column center. Therefore, the most of calculations in the present study were performed in the variant of fuel pellet injection into the plasma center. According to the calculation results the fusion power levels of about $10 \mathrm{MW}$ are reached in the system under consideration at an average plasma density value of $7 \times 10^{19}-8 \times 10^{19} \mathrm{~m}^{-3}$. Fig. 1 presents the time dependences of the DT-fusion power, when the power from the external heating sources was loaded by equal portions into ions and electrons, for two average plasma density values. In this case the injection has been carried out into the plasma center with a half width equal to the plasma column radius $(\Delta$ $=1$ ). The heating process duration in this calculation was $28.2 \mathrm{~s}$. The maximum fusion power for the plasma density of $7 \times 10^{19} \mathrm{~m}^{-3}$ was equal to $11 \mathrm{MW}$ and 13.5 MW in the second case.

The temporal behavior of average values of the electron and ion temperature, under conditions given in Fig. 1, is shown in Fig. 2. The electron temperature is slightly higher than the ion temperature. It can be explained by the influence of two processes: the $\alpha$-particle energy absorption by electrons and the energy exchange between ions and electrons when the potential difference has been overcome in the ambipolar electric field having a negative value practically throughout the plasma column cross-section (Fig. 3). Note, that at the lower value of the plasma density its temperature value is higher.

Despite the fact that the pellet evaporation region has a width equal to the plasma radius, the plasma density distribution is very peaked. A maximum value of the density in the plasma column center reaches $10^{21} \mathrm{~m}^{-3}$, while in the peripheral part of the plasma radius the density value is significantly decreased.

As the average value of the plasma density increases its maximum values in the plasma center become higher. At the same time the profiles of the ambipolar electric field value practically have no difference. Note, also, if the average plasma temperature values are less than $4 \mathrm{keV}$ the negative values of the ambipolar electric field exceed $20 \mathrm{kV} / \mathrm{m}$.

Fig. 4 illustrates the temporal behavior of the bremsstrahlung power and cyclotron radiation power for the case of the plasma density $<n>=7 \cdot 10^{19} \mathrm{~m}^{-3}$. Note that in the data of Figs. 1-4, the impurity influence

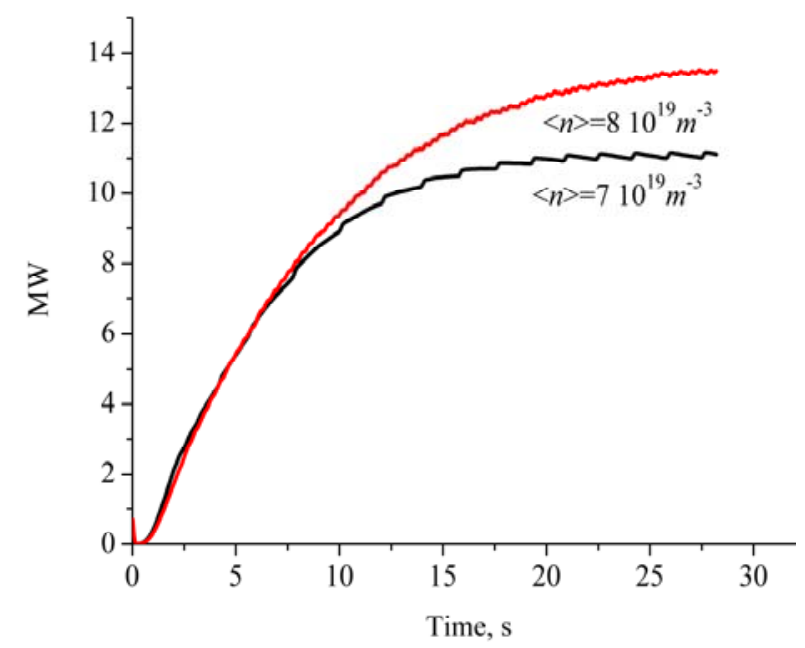

Fig. 1 Total DT-fusion power as a function of the time for two values of the plasma density, $7 \cdot 10^{19}$ and $8 \cdot 10^{19} \mathrm{~m}^{-3}$, in the case of fuel injection into the plasma center with evaporation half-width $\Delta=1$ and source heating power $P_{h i}=P_{h e}=5 \mathrm{MW}$.

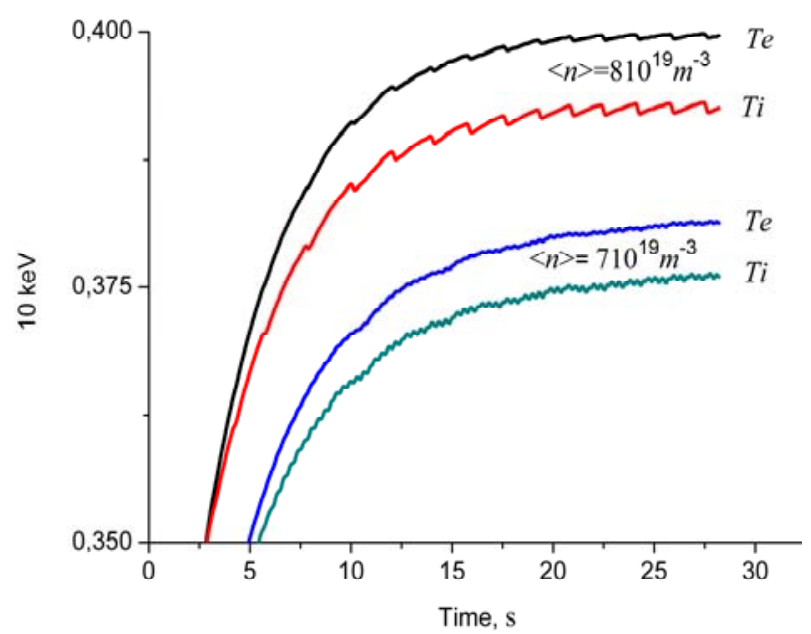

Fig. 2 Plasma component temperatures as a function of the time for two values of the plasma density, in the case of fuel injection into the plasma center with evaporation half-width $\Delta=1$ and source heating power $\boldsymbol{P}_{h i}=\boldsymbol{P}_{h e}=5 \mathrm{MW}$. 


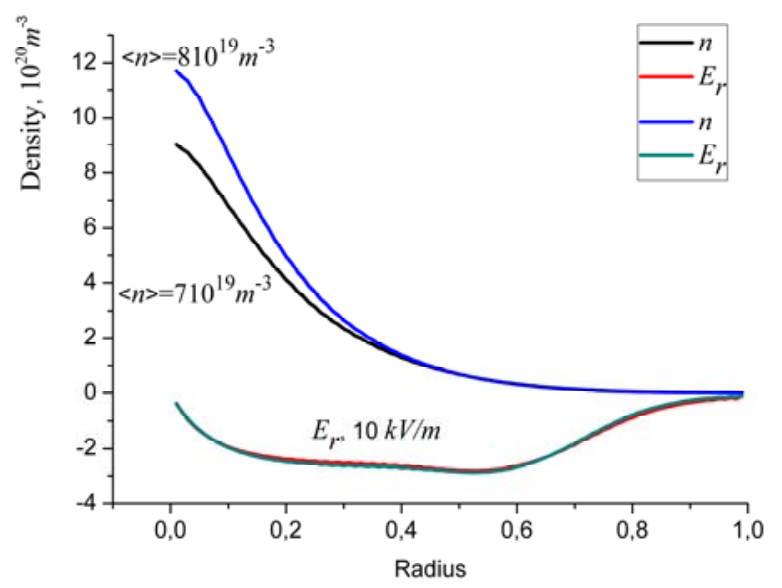

Fig. 3 Radial distributions of the plasma density and electric field for their two average values at the end of heating process $\left(P_{h i}=P_{h e}=5 \mathrm{MW}, \Delta=1, \mathrm{t}=28.2 \mathrm{~s}\right)$.

has not been taken into account. The total power of losses, caused by the bremsstrahlung and cyclotron radiation, amounts to $6 \mathrm{MW}$ that is about $50 \%$ of the total power released in the plasma (10 MW is the heating from external sources and about $2 \mathrm{MW}$ from $\alpha$-particles).

Fig. 5 shows the radial distributions of plasma ion and electron temperatures for two values of the plasma density: $7 \times 10^{19}$ and $8 \times 10^{19}$. In the central region of the plasma column the temperatures of ions and electrons practically are equal. In the peripheral part of the plasma the difference in the temperature values becomes sufficiently great. Most likely in this region the collisional heat exchange between components is insignificant because of a low density. The difference in the temperature values determines the energy exchange as a result of overcoming the potential difference created by the ambipolar electric field. The fusion powers and the plasma component temperatures as a function of the heating time at different sizes of the pellet evaporation region $\Delta$ are shown in Figs. 6 and 7.

The nuclear fusion power in the case of a small ablation region width $(\Delta=0.5)$ at a certain instant of time reaches $60 \mathrm{MW}$. Subsequent parameter realignment leads to the fusion power decrease to about 40 MW. Corresponding changes in the plasma temperature are observed in the time-temperature curves (Fig. 7). The ablation width increase leads to the plasma temperature and fusion power decrease. The difference between the temperatures of ions and electrons in each of the above-mentioned cases does not exceed $100 \mathrm{eV}$.

The results given in Figs.1-7 were obtained by the fuel pellet injection into the plasma column center with different values of the evaporation region width. The calculations show that the fuel injection into the

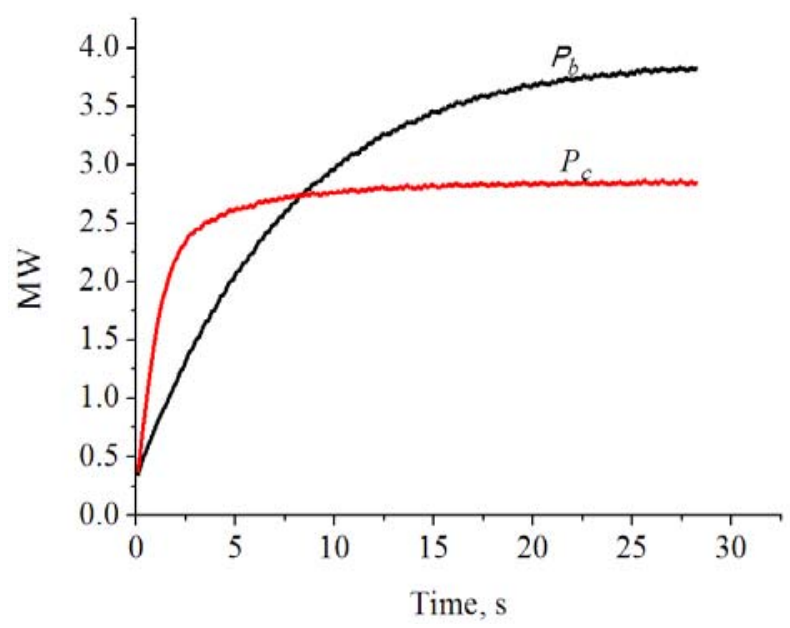

Fig. 4 Bremsstrahlung and cyclotron radiation power as a function of the heating time for the plasma density value $<\mathrm{n}>=7 \times 10^{19} \mathrm{~m}^{-3}$ in the case of fuel injection into the plasma center with evaporation half-width $\Delta=1$ and source heating power $P_{h i}=P_{h e}=5 \mathrm{MW}, \mathrm{Z}=1$.

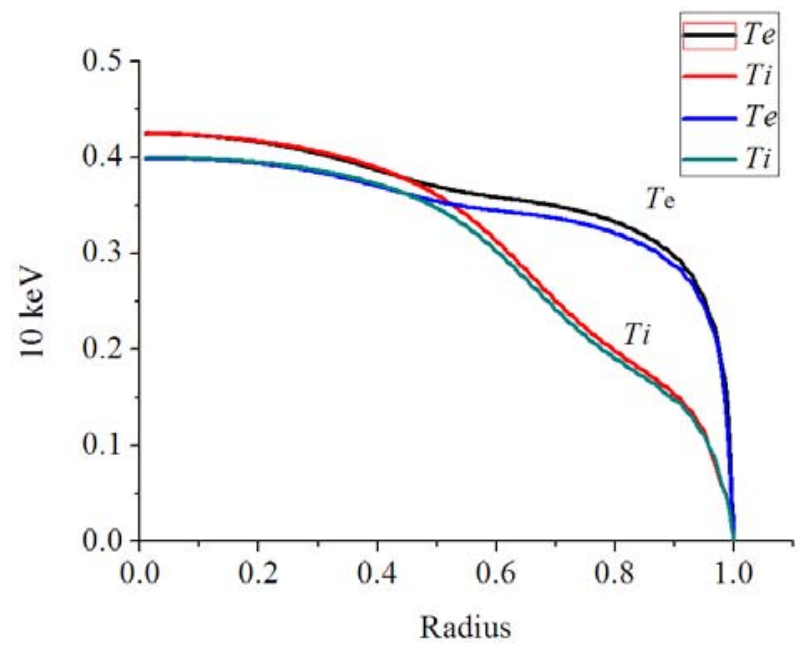

Fig. 5 Radial distributions of the plasma temperature for two average plasma density values at the end of the heating process $\left(P_{h i}=P_{h e}=5 \mathrm{MW}, \Delta=1, \mathrm{t}=28.2 \mathrm{~s} ., \mathrm{Z}=1\right)$. 


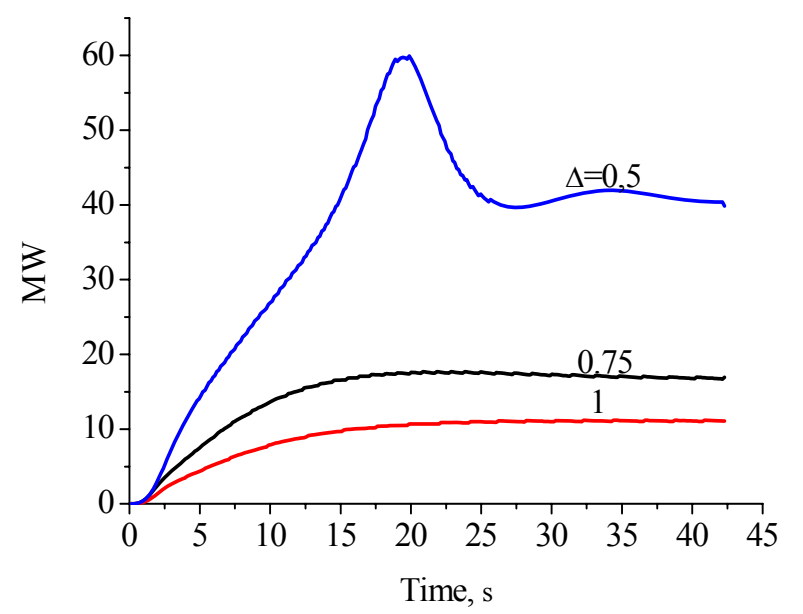

Fig. 6 DT-fusion power in the plasma heating process as a function of the time with different half-widths of the fuel pellet evaporation region $\Delta=1,0.75,0.5 .<\mathrm{n}>=0.7 \times 10^{20} \mathrm{~m}^{-3}$, $P_{h i}=P_{h e}=5 \mathrm{MW}, \mathrm{Z}=1$.

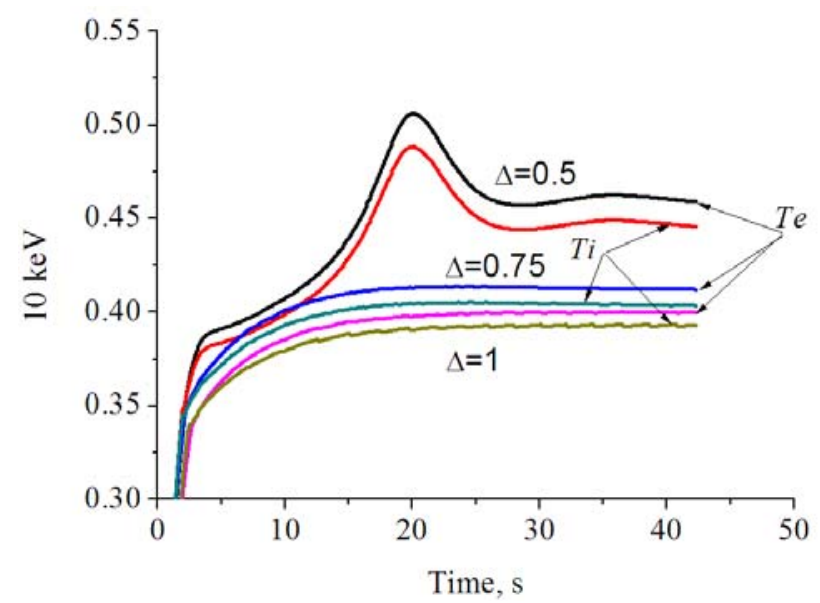

Fig. 7 Plasma component temperature as a function of the time in the plasma heating process with different half-widths of the fuel pellet evaporation region $\Delta=1,0.75$, 0.5. $<n>=0.7 \times 10^{20} \mathrm{~m}^{-3}, P_{h i}=P_{h e}=5 \mathrm{MW}, \mathrm{Z}=1$.

peripheral plasma region leads to the significant change in the spatial distribution of plasma parameters.

Figs. 8-10 demonstrate a case of fuel injection into the region of the plasma column half-radius with a half-width of the pellet evaporation region $\Delta=0.24$. In this case a maximum of the plasma density is formed in the fuel injection region with a deepening in the plasma column center. Maximum negative values of the ambipolar electric field are shifted relatively to the density maximum towards the plasma edge.

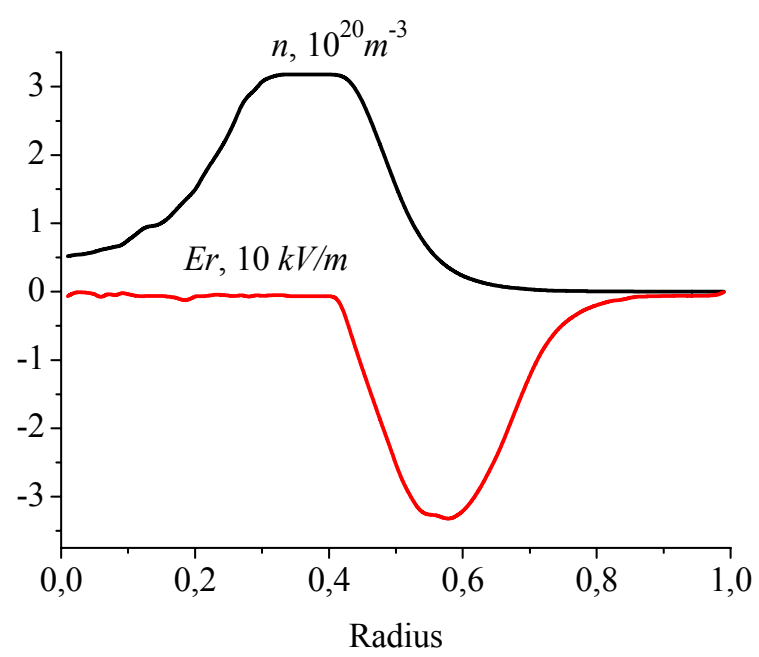

Fig. 8 Radial distributions of the plasma density and ambipolar electric field in the case of fuel injection into the region of the plasma column radius half-width $\left(x_{i}=0.5, P_{h i}=\right.$ $\left.P_{h e}=5 \mathrm{MW}, \Delta=0.24,<n>=7 \times 10^{19} \mathrm{~m}^{-3}, t=36.2 \mathrm{~s}, Z=1\right)$.

The radial distributions of ion and electron temperatures are shown in Fig. 9. In the plasma center the temperatures have a maximum, unlike the plasma density profile the maximum of which takes place in the fuel injection region. Here the ion temperature is slightly higher than the electron temperature. The temperature equalization takes place in the fuel injection region. Near the plasma edge the electron temperature is higher than the ion temperature. It is due to the energy exchange between the components in the

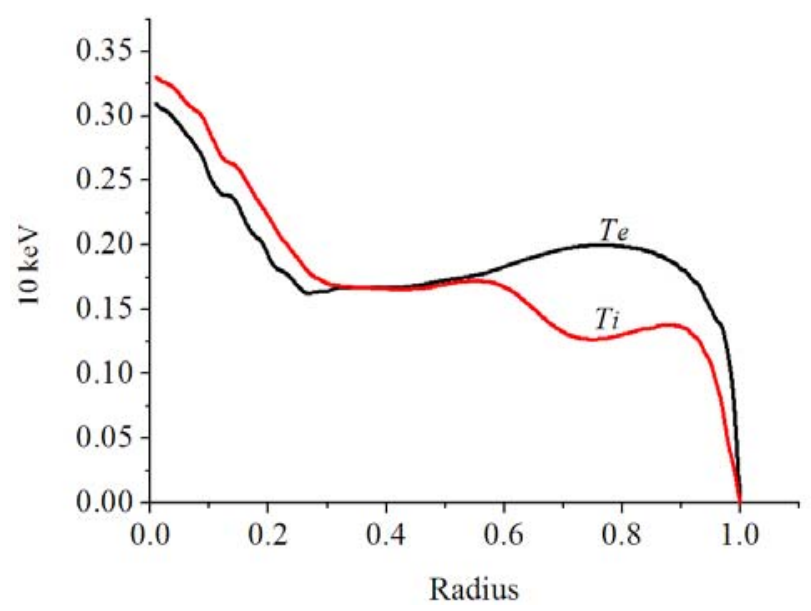

Fig. 9 Radial distribution of the plasma temperature in the case of fuel injection into the region of the plasma column radius half-width $\left(x_{i}=0.5, P_{h i}=P_{h e}=5 \mathrm{MW}, \Delta=0.24,<n>=\right.$ $\left.7 \times 10^{19} \mathrm{~m}^{-3}, t=36.2 \mathrm{~s}, Z=1\right)$. 
presence of the radial electric field.

Fig. 10 shows the distributions of bremsstrahlung and cyclotron radiation specific powers. Maxima of radiations correspond to the plasma density maximum. In the regions with a low plasma density the cyclotron radiation power is higher than the bremsstrahlung power. The ratio between the radiation power values changes to the opposite one in the region with a higher plasma density. Note that the nuclear fusion power in this variant of fuel injection is at a level of $0.1 \mathrm{MW}$.

The above-given calculation results were obtained under the assumption of the impurity absence in the plasma $(Z=1)$. Nevertheless, the bremsstrahlung withdraws a significant part (to $30 \%$ ) of the fusion power and external heating sources released in the plasma (Figs. 1-4). At the same time, the bremsstrahlung power strongly depends on the value of the plasma ion charge Z. Fig. 11 illustrates the temporal behavior of the fusion power for the cases $Z=1,1.2$ and 1.5. The calculations have been made for the plasma density $\left\langle n>=0.7 \times 10^{20} \mathrm{~m}^{-3}\right.$ and the ablation region width $\Delta=0.9$. When $\mathrm{Z}=1.2$ the fusion power with charge stationary maintenance decreases approximately by $1 \mathrm{MW}$ compared to the case with

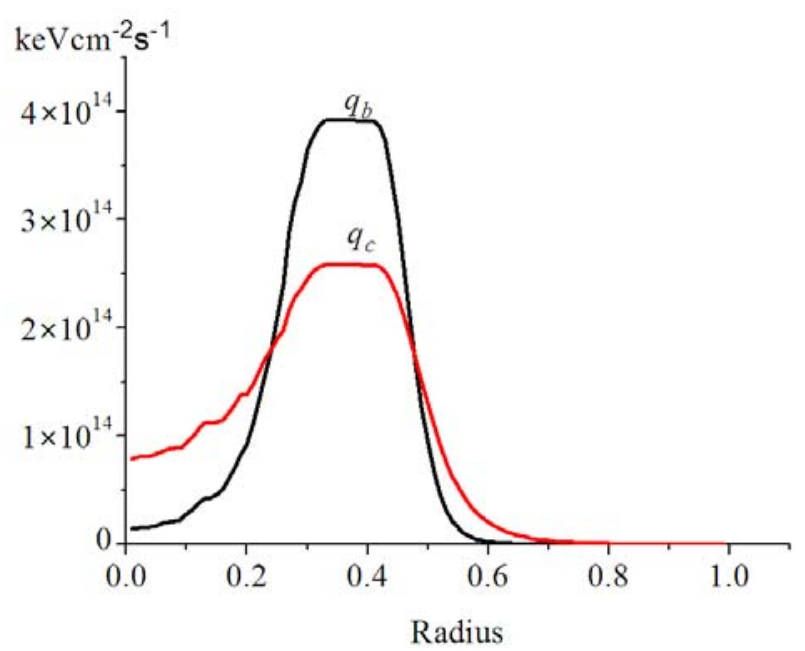

Fig. 10 Radial distributions of the specific powers of bremsstrahlung $q_{b}$ and cyclotron $q_{c}$ radiations in the case of fuel injection into the region of the plasma column radius middle part $\left(x_{i}=0.5, P_{h i}=P_{h e}=5 \mathrm{MW}, \Delta=0.24,<n>\right.$ $\left.=7 \times 10^{19} \mathrm{~m}^{-3}, t=36.2 \mathrm{~s}\right)$.

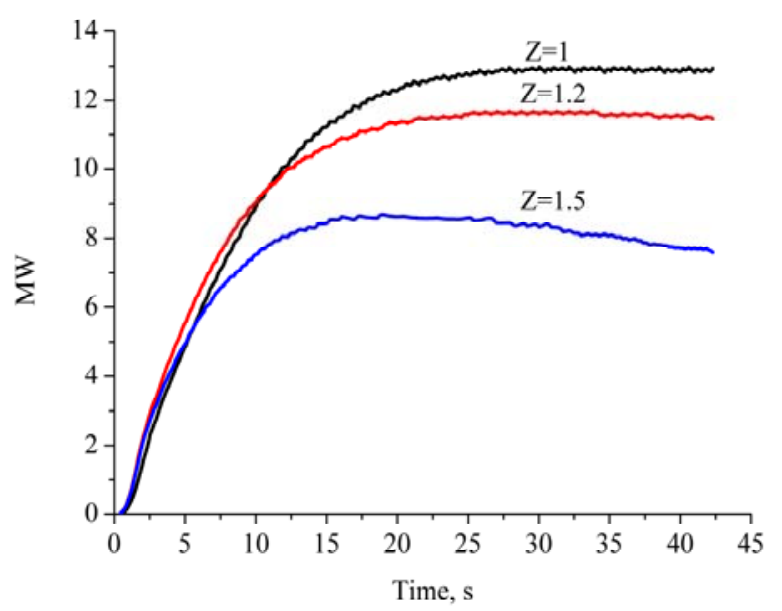

Fig. 11 DT-fusion power in the plasma heating process as a function of the time with different values of the $\mathrm{Z}$ plasma $\left(\Delta=0.9,<\mathrm{n}>=0.7 \times 10^{20} \mathrm{~m}^{-3}, P_{h i}=P_{h e}=5 \mathrm{MW}, \mathrm{Z}=1,1.2\right.$, 1.5).

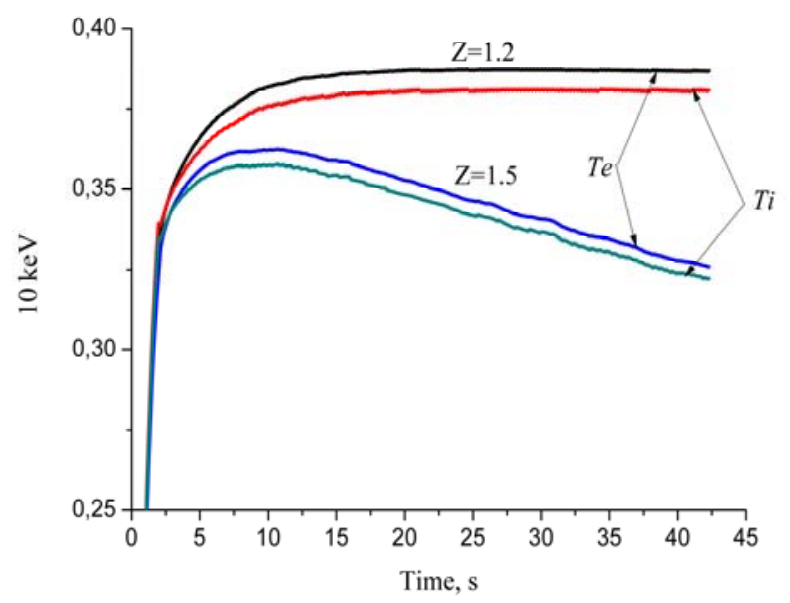

Fig. 12 Plasma component temperature as a function of the time in the plasma heating process with different values of the Z plasma $\left(\Delta=0.9,<n>=0.7 \times 10^{20} \mathrm{~m}^{-3}, P_{h i}=P_{h e}=\right.$ 5 MW).

$Z=1$. When $Z=1.5$ the charge development leads to the fusion power increase, approximately to 20th second with a maximum value near $8 \mathrm{MW}$, followed by the smooth discharge decay. As $Z=1.5$ the plasma temperature also is decreasing with time (Fig. 12). Such a plasma behavior is, probably, caused by the density profile realignment when the total loss power, with taking into account the bremsstrahlung power increase, exceeds the heating source power. At the plasma density $0.8 \times 10^{20} \mathrm{~m}^{-3}$ the discharge decay begins just at $Z=1.2$. 


\section{Discussion and Conclusions}

The possibility of neutron source development has been studied applying the stellarator system with linear dimensions increased by a factor of 1.5 compared to the experimental facility LHD. The calculations carried out under the assumption of the neoclassical transport show that using the $10 \mathrm{MW}$ plasma heating sources, it is possible to obtain plasma parameters with which in the system the nuclear fusion power from one to several tens MW is produced.

According to the investigation results the following can be concluded:

(1) Optimum results, from the point of view of obtaining a maximum fusion power output, are reached by the fuel injection into the plasma column center. Besides, a more narrow fuel pellet evaporation region, a higher fusion power.

(2) The fuel injection into the plasma center leads to the formation of strongly peaked plasma density distributions.

(3) The nuclear fusion power increases with average plasma density value increasing.

(4) The fuel injection into the peripheral region of the plasma column results in the plasma density spatial distributions, the maxima of which take place in the fuel injection region.

(5) The impurity presence in the plasma causes the discharge decay just at $Z=1.5$.

In addition, it should be noted that the formation of a strongly peaked plasma density distribution is accompanied by the gas-kinetic plasma pressure increase when the value of $\beta$ reaches more than ten percent that can create the problem for the stability of such distributions.

The study has been carried out under the assumption of the neoclassical transport. The influence of some plasma loss mechanisms was not taken into account here. Therefore the question of calculation data application to the real system depends on the existence of another, not considered here, transport mechanisms.

Furthermore, an additional study is necessary for the problem of breeding blanket location in the system under consideration.

\section{References}

[1] E. Fermi, Lectures on physics of neutrons, "Nauka" M. 2 (1972) 103.

[2] I.N. Golovin, G.E. Shatalov, B.N. Kolbasov, Some problems of hybrid reactors, News of Academy of Sciences, USSR 6 (1975) 26-34. (in Russian)

[3] I.N. Golovin, B.N. Kolbasov, V.V. Orlov, V.I. Pistunovich, G.E. Shatalov, The nuclear fuel problem and fusion-fission hybrid reactors, Vienna: IAEA, 1978, IAEA-TC-145/25.

[4] E.P. Velikhov, V.A. Glukhikh, V.V. Guryev, B.B. Kadomtsev, B.N. Kolbasov, et al., Hybrid tokamak-reactor for fission fuel production and electricity, Atomic Energy 45 (1) (1978) 3-9. (in Russian)

[5] A.V. Bazaeva, V.E. Bykov, A.V. Georgievskij, I.N. Golovin, L.V. Mikhajlovskaya, V.G. Peletminskaya,et al., Magnetic configuration of poloidal divertor tokamak, Nuclear Fusion 20 (1980) 83.

[6] P.H. Rebut, Alfen prize lecture, in: Proceeding 33-d EPS Conf. Plasma Physics, Rome, Italy, 2006.

[7] E.P. Velikhov, In: IAEA Fusion Energy Conf., Chengdu, China, 2006.

[8] Y. Wu, FDS Team, The fusion-fission hybrid reactor for energy production, In: A Practical Path to Fusion Application, 2nd IAEA Fusion Energy Conf, Geneva, Switzerland, 2008.

[9] B.V. Kuteev, V.I. Khripunov, Present-day view on a hybrid reactor, problems of atomic science and technology, Series: Thermonuclear fusion 1 (2009) 3-29.

[10] R.J. Hawryluk, W. Blanchard, M. Beer, M.G. Bell, R.E. Bell, Fusion plasma experiments on TFTR: A 20 year retrospective, Physics of Plasmas 5 (5) (1998) 1577.

[11] J. Jacquinot, V.P. Bhatnagar, J.G. Cordey, L. Horton, D.F. H. Start, R. Barnsley, et al., Overview of ITER physics deuterium-tritium experiments in JET, Nuclear Fusion 39 (2) (1999) 235

[12] E.P. Velikhov, S.V. Putvinsky, Thermonuclear reactor. Thermonuclear power. Status and role in the long-term outlook. http://www.scorcher.ru/art/science/termo/1.php. (in Russian)

[13] S.P. Hirshman, K.C. Shaing, W.I. Van Rij, C.O. Beasley, E.C. Crume, Plasma transport coefficients for nonsymmetric toroidal confinement systems, Phys. Fluids. 29 (1986) 2951.

[14] L.M. Kovrizhnykh, The energy confinement time in stellarators, Nucl. Fusion, 24 (1984) 435.

[15] D.D.-M. Ho, R.M. Kulsrud Neoclassical Transport in Stellarators, Preprint PPPL. \#2253, 1985. 
[16] H. Ehmler, Y. Turkin, C.D. Beidler, H. Maaßberg, A. Dinklage, T. Klinger, et al., Experimental check of neoclassical predictions for the radial electric field in a stellarator, Nucl. Fusion. 43 (2003) L11-L13.

[17] Y. Turkin, C.D. Beidler, H. Maaßberg, S. Murakami, V. Tribaldos, A. Wakasa, Neoclassical transport simulations for stellarators, Physics of Plasmas 18 (2011) 022505.

[18] J. Baldzuhn, M. Kick, H. Maasberg, the W7-AS Team, Measurements and calculation of the radial electric field in the stellarator W7-AS, Plasma Phys. Control. Fusion. 40 (1998) 967.

[19] J.G. Olaya, Study of electron heat transport in LHD and TJ-II, Ph.D. thesis, Universitat Politechnica de Catalunya, 2006, p. 188.

[20] A. Wakasa, S. Murakami, S. Oikawa, Study of neoclassical transport in LHD plasmas by applying the DCOM/NNW neoclassical transport database, Plasma and Fusion Research 3 (2008) 1030.

[21] V.A. Rudakov, About physical parameters of experimental reactor-stellarator in the conditions of ambipolarity of neoclassical transport fluxes, Problems of atomic science and technology, Series: Plasma Physics 18 (2012) 16-18.

[22] V.A. Rudakov, On physical parameters of the demonstration stellarator-reactor operating in the mode of self-supported thermonuclear reaction, Journal of Kharkiv National University, Physical Series: Nuclei, Particles, Fields 1017 (3/55) (2012) 66 -74.

[23] V.A. Rudakov, About parameters of reactor-stellarator in the conditions of ambipolarity of neoclassical transport fluxes, Journal of Kharkiv National University, Physical Series: Nuclei, Particles, Fields 1001 (2/54) (2012) 15-23.

[24] K. Yamazaki, M. Mikhailov, S. Sakakibarac, S. Okamura, J. Garsia, J. Dies, H. Funaba, T. Amano, Neoclassical and anomalous transport analysis of helical reactor plasmas, J. Plasma Fusion Research Series 6 (2004) 357-361.

[25] S. Murakami, A. Wakasa, H. Maaberg, C.D. Beidler, H. Yamada, K.Y. Watanabe, LHD Experimental Group, Neoclassical transport optimization of LHD, Nuclear Fusion 42 (2002) L19-L22. 


\title{
The Performance Analysis of the Wavelet-OFDM New Scheme in AWGN Channel
}

\author{
Alaa Ghaith, Rima Hatoum and Hiba Mradand Ali Alaeddine \\ Electronics and Physics Department Faculty of Sciences I, Lebanese University Beirut, Hadath 6573-14, Lebanon
}

Received: August 02, 2013 / Accepted: September 06, 2013 / Published: February 15, 2014.

\begin{abstract}
Orthogonal frequency division multiplexing (OFDM) is a special form of multi-carrier transmission that uses the policy of divide and rule. In this scheme, a large number of orthogonal, overlapping, narrow band sub-channels (subcarriers) are transmitted in parallel and divide the available transmission bandwidth. This techniqueis originally based on the Fast Fourier Transform of the information data. In order to improve the performance of the OFDM and overcome some limitations, an alternative OFDM approach based on the Wavelet Transform is proposed. In this paper, we study the performance of such systems in additive white Gaussian channel (AWGN). MATLAB simulations are realized and performance comparisons are presented.
\end{abstract}

Key words: Multi-carrier modulation, discrete wavelet transform, wavelet packet transform, Fourier-based OFDM, wavelet-based OFDM.

\section{Introduction}

In wireless communications field, there is a rising demand for high performance, high capacity and high bit rate. Digitally techniques were adopted for these purposes. OFDM is a digital modulation technique that allows transmitting high data rates over extremely hostile channels at a comparable low complexity. In recent years, it has been adopted in a wireless local and metropolitan area networks and has been used in most of popular standards such as wireless fidelity (WiFi), Worldwide Interoperability for microwave access (WiMax), Digital Video Broadcast (DVB), 3rd Generation Partnership Project/Long Time Evolution (3GPP/LTE) standards. Due to worse transmission channel conditions, the interferences could attack the transmitted signal. The OFDM technique emerged to overcome this problem and combat channel distortion. It can be viewed as using many slowly modulated

\footnotetext{
Corresponding author: Alaa Ghaith, associated professor in digital communications, research fields: digital communications, signal processing, forward error correction codes, modulation, fourth generation, beyond $4 \mathrm{G}$ and OFDM systems. E-mail: alaaghaith@ul.edu.lb.
}

narrowband signals rather than one rapidly modulated wideband signal [1]. It is a promising multi-carrier transmission technique, which divides the available spectrum into many subcarriers, each one being modulated by a low rate data stream, and then transmitted in parallel [2]. This division of the total bandwidth is an applicable way to deal with interferences that could attack the transmitted signal and to improve the spectral efficiency. Conventional OFDM is implemented using Fast Fourier Transform (FFT) and its counterpart Inverse FFT (IFFT) offering subcarriers orthogonality which prevents any expected carriers interference. FFT has a major drawback representing by high level side lobes. This leads to high interference and lower performance levels [3]. Thus, a guard interval or specifically cyclic prefix (CP) was inserted to mitigate this type of interference and to overcome the delay spread of the channel [1]. However, the CP brings a disadvantage of reducing the spectral efficiency for the transmitted signal by consuming bandwidth and power [4] without carrying additional information. Therefore, FFT based OFDM system 
presents another major problem presented by the high peak to average power ratio (PAPR).

An alternative OFDM design was proposed to overcome the limitations of the FFT based OFDM system. This is done by implementing a system with a discrete wavelet transform (DWT) instead of the FFT. The use of wavelet promises to reduce the inter-symbol-interference (ISI) and the inter-carrier-interference (ICI). It offers a high suppression of sides lobes. The Discrete Wavelet Transform is used in a variety of signal processing applications, such as video compression, object recognition and numerical analysis. Recently, the wavelet OFDM approach has been introduced into the communication field and it is included in the IEEE 1901-2010 standard. Introducing of the DWT with the OFDM is extended to another form of wavelet transform; it is the Wavelet Packet Transform (WPT). Many researches have been carried out on this domain. The performance of Discrete Fourier Transform DFT-OFDM and DWT-OFDM using Binary Phase Shift Keying (BPSK) modulation are compared in Ref. [5] on three different channel models. While the impact of constellation size of Quadrature Amplitude Modulation (QAM) over FFT-OFDM and WPT-OFDM in AWGN channel is given in Ref. [6]. The performance comparison of DFT-OFDM and DWT-OFDM using arbitrary FIR channel with different types of wavelet packets is presented in Ref. [7]. In this work, we investigate the performance of wavelet OFDM system using the Haar wavelet, due to its some motivating features, upon an AWGN channel.

The paper sections are organized as follows. Section 2 presents the conventional Fourier-based OFDM. Section 3 theoretically describes the Wavelet-based OFDM. Simulation results are discussed in section 4. Finally, we conclude and present future work in Section 5.

\section{Fourier-Based OFDM}

In the FFT-based OFDM system, the information bit stream is first mapped using common digital modulation schemes such as Phase Shift Keying (PSK) and QAM schemes at the transmitter side. In this process, the serial binary data are converted into complex symbols representing constellation points. The Fast Fourier Transform (FFT) is applied at the receiver side to decode the signal. Then, each $\mathrm{N}$ symbols will be transmitted over $\mathrm{N}$ orthogonal subcarriers through the IFFT. The output of the IFFT block represents the OFDM symbol in the continuous time domain that is stated below:

$$
x(t)=\frac{1}{\sqrt{N}} \sum_{k=0}^{N-1} X_{K} e^{2 \pi i \Delta f t} \quad 0 \leq t \leq T
$$

where, $\Delta f$ is the sub-carriers separation, $X K$ is the FFT symbol information.

After the OFDM symbol being constructed, a guard interval is added to mitigate the effect of ISI, which is caused upon propagating through the channel. Thus, a Cyclic Prefix is inserted at the end of each OFDM symbol (Fig. 1). At the receiver side, the data are reversely reconstructed using the FFT block in order to get the initial transmitted bit stream after applying the corresponding demodulation scheme. However, adding a $\mathrm{CP}$ reduces the spectral efficiency. Moreover, the high level side lobes create by the rectangular window of the FFT may cause interference in the frequency domain. These lacks have to be overcome.

\section{Wavelet-Based OFDM}

Unlike Fourier analysis which divides the whole bandwidth into orthogonal and overlapping subbands

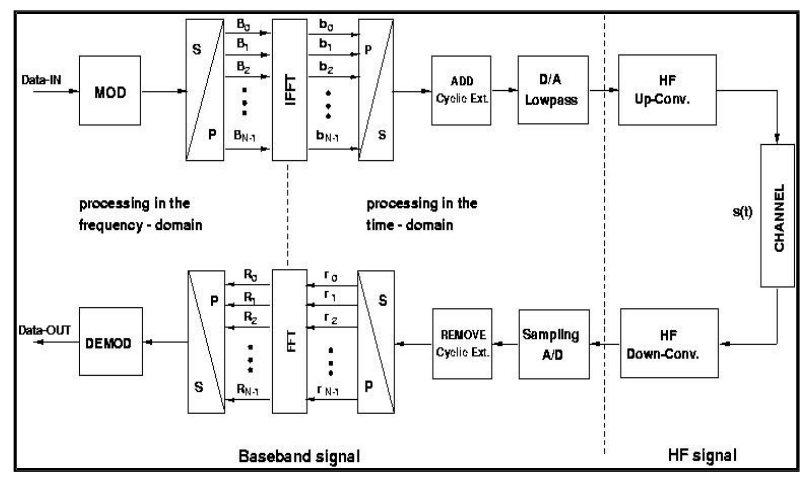

Fig. 1 An OFDM transceiver with a fast Fourier transform. 
of equal bandwidths, Wavelet transform (WT) is a multiplexing transmission method in which data is assigned to subbands having different time and frequencyresolutions. This allows a form of time-frequency analysis (or translation-scale in wavelet speaks). WT uses local orthogonal waves as basis functions to process data instead of sinusoids orthogonal basis.

\subsection{Discrete Wavelet Transform (DWT)}

The set of scaled and shifted wavelets constitutes an orthogonal basis of DWT, presenting an applicable characteristic invested in the subcarriers overlapping:

$$
\Psi_{m, n}(t)=2^{\frac{m}{2}} \psi\left(2^{m} t-n\right)
$$

where, $\psi(t)$ denotes the mother wavelet, $\mathrm{m}$ and $\mathrm{n}$ are the scale and translate parameters, respectively. We define another function $\varphi(t)$ called the scaling function or the father wavelet where the shifting and scaling of this function will cover the entire square integral real space. $\psi(t)$ and $\varphi(t)$ are used $\varphi(t)$ together to process any signal and analyze it. They are related in the following relations:

$$
\begin{gathered}
\phi(t)=\sum_{n} h_{\varphi}(n) \sqrt{2} \phi(2 t-n) \\
\psi(t)=\sum_{n} h_{\psi}(n) \sqrt{2} \phi(2 t-n)
\end{gathered}
$$

$h_{\phi}(n)$ and $h_{\psi}(n)$ are respectively a low pass and a high pass filter banks. The low pass filter gives approximate information about the signal while the high pass filter produces detailed information. We mainly interest in the approximate coefficients where we can find most of the information. Fig. 2 shows the process done with DWT filter banks. The low or approximate coefficients are down sampled and re-filtered.

The signal reconstruction is done in the same way by filtering the coefficients with the same filter banks used for decomposition-Note that both filters are also assumed to have perfect reconstruction property, this means that the input and output of the two filters are expected to be the same [8]. Finally, they are up-sampled to get the original signal.

\subsection{Wavelet Packet Transform (WPT)}

In the DWT, only the approximate coefficients are decomposed again and decimated by 2 . WPT both approximate and detailed coefficients undergo the same operation of decomposition and decimation at each level [3]. For the wavelet packet, we can decide how to decompose the high and low frequency parts, after each decomposition, we can decide whether to decompose the signal in the low/high frequency domain or not. They form basis, which retain many of the orthogonality, smoothness, flexibility, and localization properties of their parent wavelets.

\subsection{Wavelet Transform-Based OFDM}

Wavelet localization feature can provide much lower side lobes magnitude than those of Fourier transform (FT) and the loss of orthogonality leads to lesser ISI and ICI. Therefore, OFDM requires time and frequency synchronization to get a good performance. This can be overcome by the use of the WT-based OFDM approach.

The FFT block in the original diagram is now replaced by the DWT block. The symbols resulted from constellation mapping undergo inverse discrete wavelet transform (IDWT) operations and construct the desired OFDM signal. The operation is done by the aid of quadrature mirror filters that are the low pass

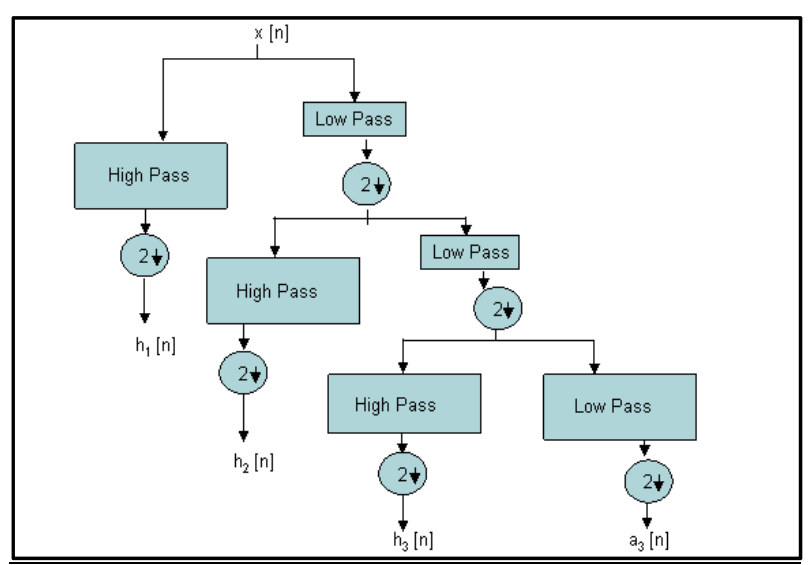

Fig. 2 Schematic diagram of a discrete wavelet transform. 
and high pass IDWT filter banks. Perfect reconstruction and orthonormal basis properties are satisfied, where symbols are convoluted with those filters and then multiplexed together forming the signal. The data are pointed as the approximate coefficients of the filters and the detailed coefficients are zeropadded [8]. At the receiver where the DWT block replaces the FFT one, the signal is reversely decomposed producing the approximate and the detailed coefficients. Actually, the useful information is contained in the approximate coefficients. QAM demodulation is then done and the data stream is retained.

Similar to IFFT, IDWT matrix is a transpose of the original. Both transforms can be viewed as a rotation in function space to a different domain [5]. For the FFT, this new domain contains basis functions that are sines and cosines. For the wavelet transform, this new domain contains more complicated basis functions called wavelets, mother wavelets, or analyzing wavelets.

Thus, an improved performance must occurwhen the wavelet transform is introduced within the multi-carrier OFDM-based system. However, the logarithmic division of the bandwidth in the wavelet transform is not well suited for OFDM multi-carrier communication [9]. This motivates us to introduce the WPT. In addition, WPT is more robust to the tone and impulse interference added into a transmission packet. So the transmission throughput is kept in WPT even in these severe interference environments [10]. However, while the DWT is less computationally complex than the FFT due to the logarithmic division of frequency, WPT is more complicated than FFT. This is the price that must be paid.

\section{Simulations Results and Discussions}

Theoretically, WT promises an improved performance in comparison with the FFT. This fact has to be exploited for the OFDM systems. However, this idea is still incompletely studied and it needs to be proved in practice. Thus, we are motivated to compare the performance of the FFT-based OFDM system with the DWT-based OFDM one. The MATLAB simulations were done to achieve this goal.

Firstly, the conventional FFT-based OFDM block diagram is designed using 128 subcarriers. Then the Discrete Fourier Transform (DFT) is replaced by the DWT. As the Mother Wavelet, the HAAR wavelet is used. The input data stream is modulated with the 16-QAM (Quadrature Amplitude Modulation). The channel is assumed as Additive White Gaussian Noise (AWGN) channel. The Bit Error Rate (BER) and the Power Spectral Density (PSD) are the main keys used to comparison. It is important to note that the choice of the wavelet transform is very critical since it is related to the orthogonality property. For some wavelet functions, bases are not orthogonal to each other. This causes the AWGN to be correlated within a subcarrier and an AWGN channel does not remain an AWGN one. Thus, the result is worse than the single carrier case. For the Haar wavelet, it is not the case and no timing frequency synchronization is done in Ref. [9]. Moreover, it is more adapted to reduce the PAPR [10].

\subsection{Bit Error Rate Performance Criterion}

The BERs of the OFDM system transmission are calculated over a several Monte Carlo runs and are plotted with respect to the signal to noise ratio (SNR) or $E_{b} / N_{0}(d B)$. Table 1 represents the parameter values used for simulation.

Fig. 3 shows the performance comparison between FFT and DWT OFDM system. We can clearly observethat the DWT based system outperforms the FFT one and provides a " $3 \mathrm{~dB}$ " performance gain.

Table 1 System parameters.

\begin{tabular}{lll}
\hline \multirow{2}{*}{ Variable } & FFT-OFDM & DWT-OFDM \\
\cline { 2 - 3 } & Matrix value & Matrix value \\
\hline Numberofsubcarriers & 128 & 128 \\
CP & 26 & 0 \\
Bitstream & 512 & 512 \\
Repetitiontimes & 20,000 & 20,000 \\
Waveletused & - & Haar \\
SNRrange $(\mathrm{dB})$ & $1-20$ & $1-20$ \\
\hline
\end{tabular}




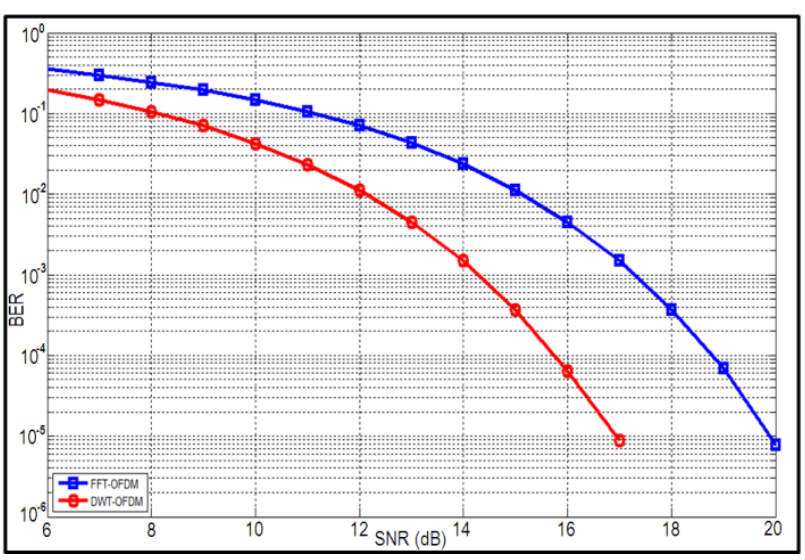

Fig. 3 FFT-based OFDM vs DWT-based OFDM.

In order to observe the effect of the OFDM system, we compare the raw 16-QAM system to the 16-QAM OFDM system. First, using the FFT tool (Fig. 4), and then using the DWT (Fig. 5). The results show that the 16-QAM DWT-based OFDM system outperforms the raw 16-QAM one with a $3 \mathrm{~dB}$ as a gain, unlike in the case of the FFT-based OFDM system where the performance is too close to the raw-QAM system. We must note here that the main cause of this observation is that we assume the AWGN channel case which does not reflect clearly the OFDM benefits. But, we focus in this work on the effect of the WT with comparison to FT within the OFDM systems.

Therefore, we can say that either QAM alone or QAM with FFT-based OFDM will do the same work under same conditions and both are less applicable when compared to DWT based OFDM system.

\subsection{Power Spectral Density Comparison}

Now, we compare in the frequency domain. The PSD for each transmitted signal is presented in Fig. 6 . QAM is theoretically a single carrier modulation scheme, where the signal is transmitted over one single frequency. Thus, the spectrum of the 16-QAM should be a single carrier signal as shown by simulation (Fig. 6). Merging QAM with OFDM creates a multi-carrier transmission. Indeed, the FFT-based OFDM spectrum proves that, as shown in Fig. 6 where the variation of the power levels indicates the separation between the sub-channels.

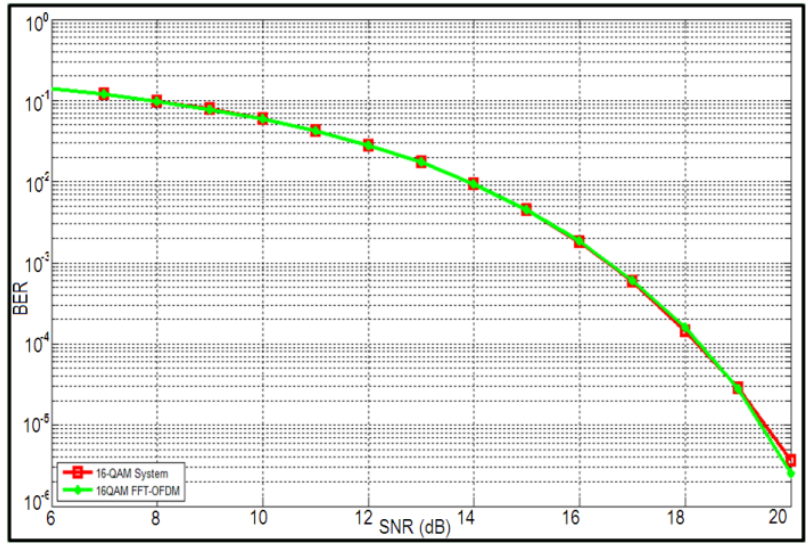

Fig. 4 16-QAM and 16-QAM with FFT-based OFDM.

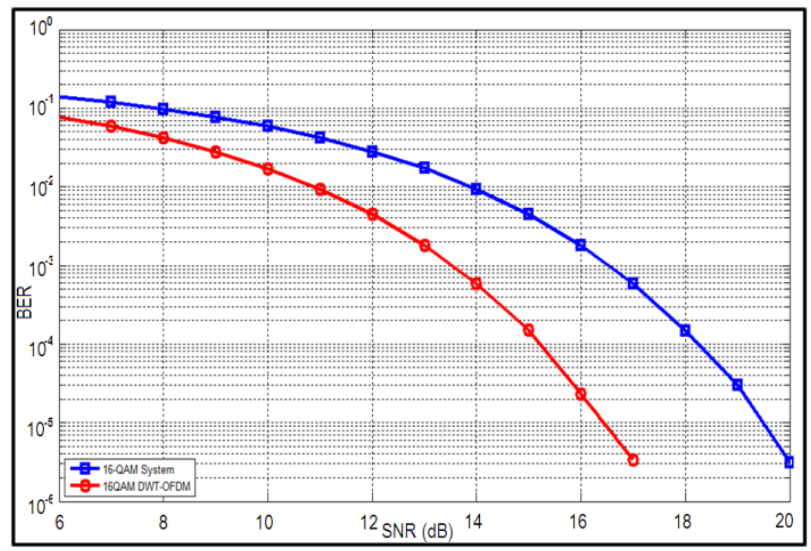

Fig. 5 16-QAM and 16-QAM with DWT-based OFDM.

Now, when substituting the FFT with DWT, as we proposed in this project, we should maintain the OFDM multi-carrier principle. This is theoretically achieved and the studies about Wavelet Transform confirm this concept. Mathematically, we can prove that the use of IDWT leads to a multi-carrier transmission.

The operation undergoes a set of translations and scales of the wavelet function along the whole signal and convolving the scaled and shifted wavelet with our concerned signal. This leads to a set of subcarriers orthogonally spaced [11]. As a result, the PSD for both FFT-based system and DWT based one should logically have the same form which meets with the OFDM theoretical one. Well, this is the main concept, but does this really happen with the MatLab simulations?

Unfortunately, simulations do not show a coincidence of the DWT-based OFDM spectrum 


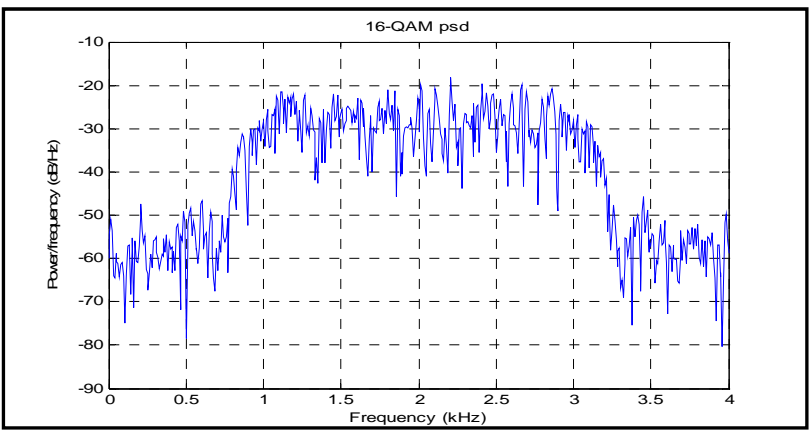

(a)

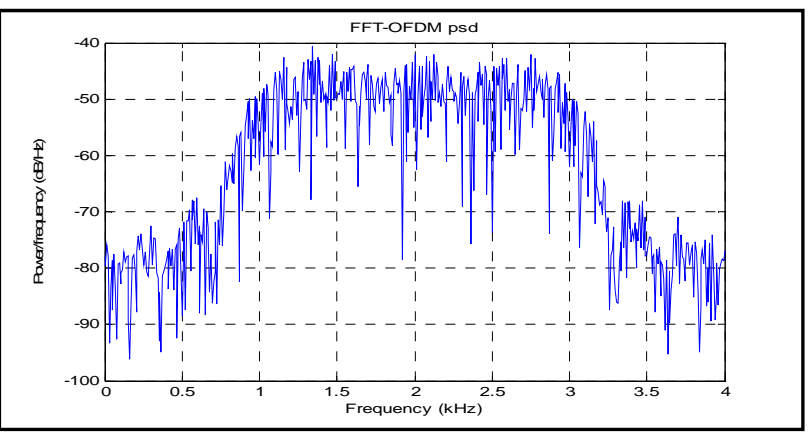

(b)

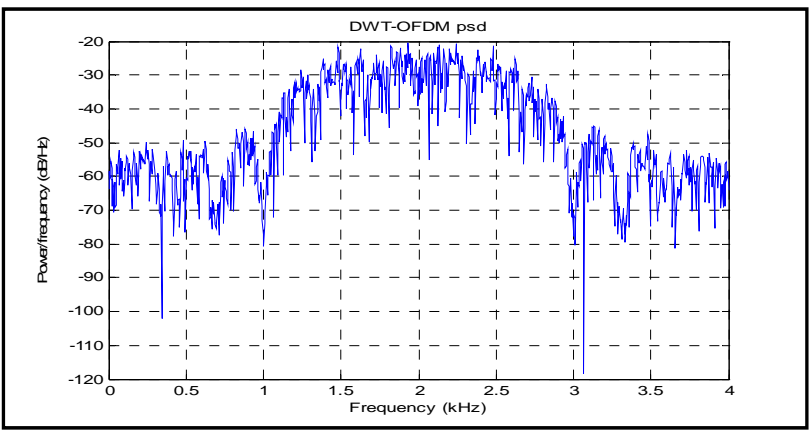

(c)

Fig. 6 Power spectral density of (a) QAM; (b) FFT-based OFDM and (c) DWT-based OFDM system.

(Fig. 6c) with the FFT-based one (Fig. 6b). Therefore, this PSD seems different from the theoretical spectrum and appears as a single carrier transmission. Otherwise, the figure shows a gain in the spectral efficiency offered by introducing of DWT: the PSD width of the DWT-based OFDM is less than that of the FFT-based OFDM since they are not adding a CP.

In fact, with Mat Lab " $D W T$ ", the convolution between the wavelet function and the data symbols is done only one time, nor shifting neither scaling are applied on the wavelet. This means that the principle of multi-carrier modulation is not applied since the reason behind the creation of subcarriers is not verified.

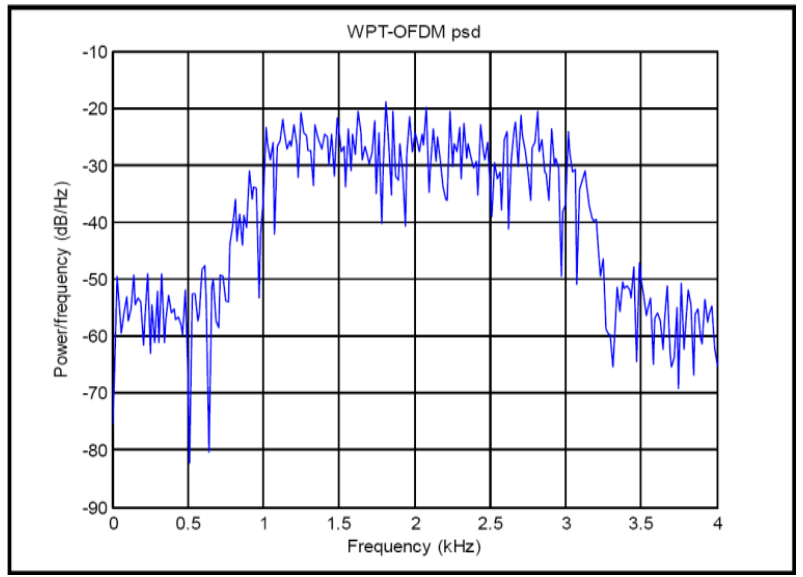

Fig. 7 Power spectral density of WPT based OFDM system.

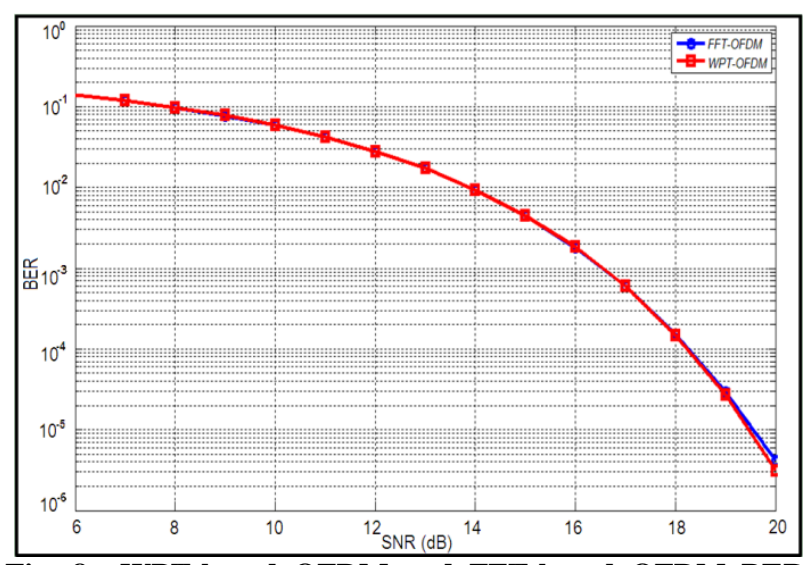

Fig. 8 WPT-based OFDM and FFT-based OFDM BER performance.

Thus, MatLab is not the right way to get the exact answer for the comparison between FFT-based OFDM and DWT-based OFDM since the first one is a multi-carrier modulation type and the second one is a single carrier. Raw QAM and DWT-OFDM can be compared; they are both a single carrier modulation types.

\subsection{Wavelet Packet Transform (WPT) Performance}

Using the 'DWT' function does not lead to a multi-carrier OFDM system. An alternative way can overcome this lack: this is the WPT. Analytically, the WPT is the Real Discrete Wavelet Transform (DWT). This MATLAB function leads to multi-carrier OFDM system. Thus, we must obtain the same PSD of the traditional OFDM system. The simulation proves this theoretical fact, as shown in Fig. 7. To reach a complete 
answer about this proposed solution we calculate the BER under the same conditions and we obtain the Fig. 8. As it is clear, the WPT has the same performance of the FFT-OFDM system and does not provide any gain.

\section{Conclusions and Future Work}

The aim of this work is improving the OFDM-transmission performance by introducing the DWT mathematical tool instead of the FFT one. Indeed, the Wavelet Transform outperforms the Fourier transform in many communication applications. MATLAB simulations show that this idea provides " $3 \mathrm{~dB}$ " in term of BER gain. However, the system lost his multi-carrier characteristic. This observation motivates us to introduce the Wavelet Packet Transform WPT as an alternative solution. This way leads to a multi-carrier OFDM system, but does not present any performance gain by comparing to the traditional OFDM system. This result leads us to a conclusion that the comparison of FFT-based OFDM and WT-based OFDM using MATLAB is not the right way to get the exact answer for our proposal. It is better wise to compare raw QAM with the DWT system; they are both a single carrier modulation.

We propose to perform this study using another simulation way to meet results with the theoretical properties. As practical case, it is suitable to study the case of the fading transmission channel.

\section{References}

[1] P. Vyas, S. Bohra, A. Parihar, I. Gupta, R. Jain, A comparison study for BER performance in OFDM systems based on using M-QAM with Detection techniques, International Journal of Advanced Technology \&
Engineering Research (IJATER) 3 (2) (2013).

[2] H. Liu, G. Li, OFDM-based broadband Wireless Networks: Design and Optimization, Chapter 2: OFDM Fundamentals, ISBN: 978-0-471-72346-2, John Wiley and Sons, 2005.

[3] V.M. Band, M.N.S. Swamy, Design \&implementation of optimized DMWT architecture for OFDM on FPGA, in: International Conference on Innovations in Electrical and Electronics Engineering (ICIEE'2012) Dubai (UAE), 2012.

[4] M. Rodrigues, Orthogonal frequency division multiplexing (OFDM): A Primer, Lecture in the Laboratory for Communication Engineering, Department of Engineering, University of Cambridge.

[5] H. Zhang, D. Yuan, M. Jiang, D. Wu, Research of DFT-OFDM and DWT-OFDM on different transmission scenarios, in: Proc. ICITA’04, 2004, pp. 31-33.

[6] H.J. Taha, M.F.M. Salleh, Performance analysis of QAM-modulation parameters on Wavelet Packet Transform (WPT) and FFT-OFDM system, in: Proc IEEE-Malaysia International Conference on Communications'09, 2009.

[7] F. Farrukh, S. Baig, M.J. Mughal, Performance comparison of DFT-OFDM and Wavelet-OFDM with zero-forcing equalizer for FIR channel equalization, in: Proc. International Conference Electrical Engineering, ICEE'07, 2007, pp. 1-5.

[8] B.G. Negash, H. Nikookar, Wavelet based OFDM for wireless channels, in: Vehicular Technology Conference, VTC 2001 Spring, IEEE VTS 53rd (Volume:1), 2001.

[9] A. Li, W. Shieh, R.S. Tucker, Wavelet packet transform-based OFDM for optical communications, Journal of Light Wave Technology 28 (24) (2010) 3519-3528.

[10] H. Zhang, D. Yuan, M. PÄtzold, Novel study on PAPRs reduction in wavelet-based multicarrier modulation systems, Digital Signal Processing ELSEVIER 17 (2007) 272-279.

[11] W. Saad, N. El-Fishawy, S. El-Rabaie, M. Shokair, An Efficient Technique for OFDM System Using Discrete Wavelet Transform, Springer-Verlag, Berlin Heidelberg, 2010, pp. 533-541. 


\title{
Enhancement of the Photodynamic Therapy Effect on
} Streptococcus Mutans Biofilm

\author{
Vitor Hugo Panhóca ${ }^{1,2}$, Mariana Carreira Geralde ${ }^{2}$, Thaila Quatrini Corrêa ${ }^{2}$, Mariana Torres Carvalho ${ }^{1}$, Clóvis \\ Wesley Oliveira Souza ${ }^{2}$ and Vanderlei Salvador Bagnato ${ }^{1}$ \\ 1. Optical Group, Biophotonics Laboratory, Physics Institute of São Carlos (IFSC), University of São Paulo (USP), São Carlos, SP \\ 13560-970, Brazil \\ 2. Biotechnology Pos-Graduate Program, Federal University of São Carlos (UFSCar), São Carlos, SP 13560-000, Brazil
}

Received: November 07, 2013 / Accepted: October 14, 2013 / Published: February 15, 2014.

\begin{abstract}
Biofilm is a community of bacteria, less susceptible to traditions treatments. Although photodynamic therapy (PDT) is a very effective way to microorganism inactivation, in biofilm it is not as efficient as it is in planktonic bacteria cultures. The increment of an element to increase the effectiveness of PDT was our aim. Therefore, this in vitro study evaluates the susceptibility of a biofilm formed by Streptococcus mutans on metallic surface of orthodontic accessories under the application of PDT with a surfactant. Samples obtained from blades of orthodontic bands ( $\mathrm{NiCr}$ ), where used as adhesion surface for the biofilm. They were treated with $1 \mathrm{mg} / \mathrm{ml}$ of curcumin, with $0.1 \%$ of sodium dodecyl sulfate and exposed to $30 \mathrm{~J} / \mathrm{cm}^{2}$ of light (455 nm). Eight experimental groups were studied, including the positive and negative controls. The results show that the group with PDT and surfactant had a significant decrease (p $<$ $0.001)$ in viability. In this case, the reduction observed was of $5.6 \log 10(\mathrm{CFU} / \mathrm{ml})$ in comparison to the control group. We have shown that, even though the biofilm is very tough and complex structure, we are able to promote almost the complete inactivation of $S$. mutans in systems similar to an orthodontic treated patient's mouth.
\end{abstract}

Key words: Photodynamic therapy, light-induced damage, oral biofilm, streptococcus mutans, curcumin, surfactant.

\section{Introduction}

There are two forms for bacteria to survive in their natural environment. They may be free-floating, as a planktonic cell, or attached to a surface, as in a biofilm. The biofilm is a complex community in which most of bacteria live. It is a well-organized community that adheres to surfaces and is embedded in an extracellular adherent layer. Bacterial resistance increases when they are organized as biofilm. Also, once in a biofilm, the bacteria display different characteristics from those that they had as a free-floating organism. Biofilms can be found in many places, and the human mouth has perfect conditions for the bacteria to grow.

Corresponding author: Vitor Hugo Panhóca, M.S., research fields: biological physics, biophotonics and dentistry. E-mail: vhpanhoca@msn.com.
For that reason, biofilms are precursor for most common oral diseases, such as caries and periodontal disease. Prior to the formation of the biofilm, a thin microorganism layer adheres on the surface of the tooth or gum. These microorganisms use salivary proteins as substrate for adhesion. Streptococcus mutans produces adhesins that together with these proteins are used to form the substrate of adhesion. The extracellular substrates that they produce allow the accession of other types of microorganism to form the mature biofilm, and finally the dental plaque.

The dental biofilm can be categorized into sub- and supra-gingival. It is formed subsequent to an increase of saccharolytic and acidogenic microorganisms, such as Streptococcus mutans and lactobacillus, which occur in most people who eat sucrose-rich food. Hence, these biofilms produce acids that cause tooth 
demineralization and caries. Four components are needed to create the carious lesion [1, 2]: a host, high-carbohydrate diet, microorganisms and time.

The Streptococcus mutans, that may be one of the most common bacteria in the oral cavity, produces a polyhedral matrix to structure the biofilm. The microorganisms existent in a biofilm become sheltered by this polyhedral matrix, reducing the action of antimicrobial agents. Compared to planktonic cells, the biofilm structure protects the bacteria, and other microorganisms. The thickness of this structure prevents contact between antimicrobial agents and microorganisms, keeping the chemicals away from the deeper layers of the biofilm. As a result, the biofilm boosts the growth rate of the microorganisms [2-4]. Consequently, microorganisms embedded in biofilms are 10 to 1000 times more resistant to antimicrobial agents $[5,6]$.

One cause that enhances the biofilm formation in the mouth is the installation of orthodontic accessories [7]. The usage of orthodontic accessories creates new retention surfaces for microorganisms in the oral cavity, increasing their retention. Therefore, it increases biofilm formation and the amount of bacteria in the mouth [7]. The high level of S. mutans in the oral cavity of patients with orthodontic accessories, compared to non-orthodontic treatment patients, is cause both by the presence of the accessories and also by the poor hygiene control of it by the patient.

Photodynamic therapy (PDT) is a well-known medical therapy for cell inactivation and microorganism control $[8,9]$. This technique is based on the combined use of light and some types of drugs, called photosensitizers (PS), which produce an oxidation reaction. This oxidative reaction can them cause the death or inactivation of selected cells. PDT can also be used as a therapy with a potent antimicrobial effect [10]. Inactivation of microorganisms by PDT can ensure several advantages over the traditional use of antimicrobials. First, bacterial killing is rapid, reducing the need to maintain high concentrations of chemicals for long periods, as in the case of antibiotics and antiseptics usage [11]. Secondly, bacterial killing is not linked to the intervention of chemicals, so, development of resistance is improbable to happen [12]. Finally, since neither the PS, nor the light, are bactericides by themselves, the destruction of bacteria can be controlled by restricting the irradiated region, preventing the destruction of the normal micro-biota elsewhere.

PDT is an alternative therapy for preventing and treating dental caries and periodontal diseases. It is an innovative way to control the formation of the bacterial biofilm, controlling the incidence of these pathogens. Most studies present high efficiency of PDT to planktonic bacteria. On the other hand, since the biofilm protects its microorganisms, the results over clinical trials are not as efficient [13]. Therefore, researches over systems that emulate the patients' mouth are so important to find the "perfect therapy". Thus, the purpose of this paper is to evaluate the effect of the antimicrobial activity of PDT on biofilms formed on orthodontic accessories. This in vitro study evaluates the use of PDT and a surfactant on metal surfaces, observing the susceptibility of biofilms formed by $S$. mutans. Our objective is to increase the performance of PDT, adding one element to the treatment, in a system that emulates the mouth real environment.

\section{Material and Method}

For this study 24 metal samples measuring $5 \times 5$ mm were used, obtained from orthodontic band blades (NiCr). On the metal surface, S. mutans biofilms were growth and threated with PDT and a surfactant. The PDT was induced by curcumin and light emitting diode (LED) in the blue range, and the surfactant used was the sodium dodecyl sulfate (SDS). There were tested eight experimental groups, each test repeated three times and each solution measured twice. 


\subsection{Biofilm Growth and CFU Preparation}

For Streptococcus mutans biofilm, an inoculum from stock culture (ATCC 25175) was grown in Brain Heart Infusion (BHI) broth (Difco, Detroit, USA) and incubated at $37{ }^{\circ} \mathrm{C}$ for $24 \mathrm{~h}$. The suspensions were centrifuged, 3,000 rpm per $15 \mathrm{~min}$ (Excelsa II centrifuge, model 206-BL, FANEM), and the bacterial pellet was dispersed into BHI broth with $20 \%$ sucrose. To archive the desired population density, the culture was adjusted to obtain standardized suspension containing $10^{6}$ cells $/ \mathrm{ml}$, the optical density of the final suspensions was verified using a digital spectrophotometer (FEMTO 600).

The biofilm was grown in orthodontic band metal blades (orthodontic appliance, Tecnident, São Carlos, Brazil), cut into $5 \times 5 \mathrm{~mm}$ squares, and sterilized in autoclave. Each sample was placed inside individuals wells of a 24-well microtiter plate. Then, aliquots of $1 \mathrm{~mL}$ of the $S$. mutans were transferred to the plates. The biofilms were incubated at $37^{\circ} \mathrm{C}$ up to 7 days, with the growth medium changed every two days. All the samples were washed in PBS solution and placed in a new well into the 24 microtiter plate to be prepared for experimental treatment. They were separated between eighth groups and treated accordingly.

After treatment, the samples and theirs solutions were transferred to eppendorfs, $1 \mathrm{ml}$ of PBS was added, and they were homogenized, to break and loosen the biofilm into the solution, during $8 \mathrm{~min}$ in an ultrasound shaker (150 Watts, $50 \mathrm{~Hz}$ ), before the metal squares were removed. The solutions were diluted up to $10^{-5}$ and uniformly spread to petri dishes containing culture medium BHI AGAR (Difco, Detroit, USA) plus $20 \%$ sucrose. They were aerobically cultured at $37{ }^{\circ} \mathrm{C}$ for $48 \mathrm{~h}$ prior to the colony-forming unit (CFU) count.

\subsection{Experimental Treatment Procedure}

The experimental groups included: control group (P-L-S-); light (P-L+S-), surfactant (P-L-S+) and photosensitizer (P+L-S-) groups; surfactant-light
$(\mathrm{P}-\mathrm{L}+\mathrm{S}+)$, photosensitizer-surfactant $(\mathrm{P}+\mathrm{L}-\mathrm{S}+)$ and PDT (P+L+S-) groups; and the PDT-surfactant $(\mathrm{P}+\mathrm{L}+\mathrm{S}+)$ group. Each group was named accordingly to the addiction of the photosensitizer ( $\mathrm{P}+$ or $\mathrm{P}-)$, surfactant (S+ or S-) and application of light ( $\mathrm{L}+$ or $\mathrm{L}-$ ).

All the samples were placed in a new well into the 24 microtiter plate and received the correct solution necessary for treatment. To the control (P-L-S-) and light (P-L+S-) groups, there was added $1 \mathrm{ml}$ of a solution of PBS with 5\% of DMSO. There was added $1 \mathrm{ml}$ of surfactant solution to the surfactant (P-L-S+) and surfactant-light $(\mathrm{P}-\mathrm{L}+\mathrm{S}+)$ groups. The surfactant solution was made in PSB with $0.1 \%$ of SDS and $5 \%$ of DMSO. To the photosensitizer (P+L-S-) and PDT $(\mathrm{P}+\mathrm{L}+\mathrm{S}-)$ groups it was added $1 \mathrm{~mL}$ of a photosensitizer solution. This solution had $1 \mathrm{mg} / \mathrm{ml}$ of curcumin, and was made in PBS with 5\% of DMSO. And to the photosensitizer-surfactant $(\mathrm{P}+\mathrm{L}-\mathrm{S}+)$ and PDT-surfactant $(\mathrm{P}+\mathrm{L}+\mathrm{S}+)$ groups, there was added 1 $\mathrm{mL}$ of a photosensitizer-surfactant solution, which had $1 \mathrm{mg} / \mathrm{mL}$ of curcumin, $0.1 \%$ of surfactant, and $5 \%$ of DMSO in PBS.

All the experimental groups were kept in the dark for $5 \mathrm{~min}$, incubation of the PS, before the application of light in the designated groups. The experimental groups with light $(\mathrm{L}+)$ were then irradiated in a home-made blue LED device (high power royal blue LEDs, 355mW, centered at $455 \mathrm{~nm}$ with $20 \mathrm{~nm}$ bandwidth), for $10 \mathrm{~min}$ and $54 \mathrm{~s}$, ensuring a $30 \mathrm{~J} / \mathrm{cm}^{2}$ dose at all the wells. The LED device (Fig. 1), called Biotable (LAT-IFSC-USP), was made to guarantee uniform distribution of light ( $46 \mathrm{~mW} / \mathrm{cm}^{2}$ intensity) in the plane were the samples were placed. The experimental groups (L-) where no light was applied were kept in the dark at room temperature, for the same amount of time.

\subsection{Data Analysis}

The effect of the photodynamic therapy and surfactant on the biofilms was evaluated by counting colony-forming units. The results were statistically evaluated by ANOVA, differences were considered 

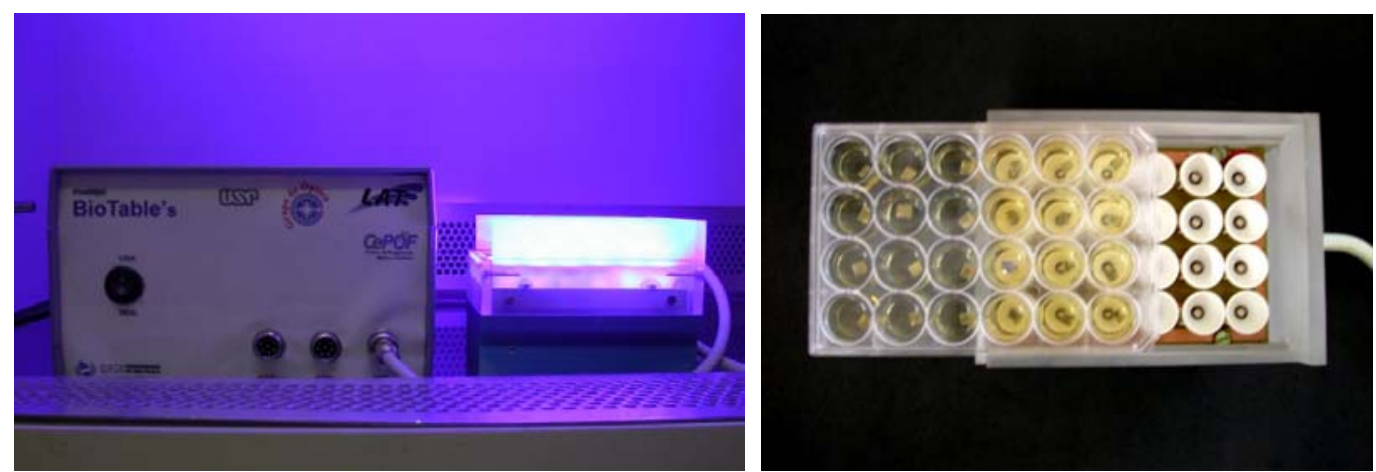

Fig. 1 Photography of the 24-well blue Biotable and details of the metal plate samples, placed in a 24-well microtiter plate, being put in place on the LED device.

when $\mathrm{p}<0.05$. The difference between treated groups with the control group (P-L-S-) was made with the OneWay ANOVA with post hoc Turkey.

\section{Results and Discussion}

To ensure biofilm formation, selected metallic samples were imaged with confocal microscopy (Fig. 2). The images showed the formation of $S$. mutans biofilm after 7 days of cultivation on the metallic samples of orthodontic appliance (NiCr). The photodynamic inactivation was also observed, by means of a live-dead biological marker, imaging plate before and after PDT treatment. The confocal reflection images show the metal surface, indicating the presence of colonies of $S$. mutans. The confocal fluorescence images show the fluorescence of the propidium iodide (PI) dye and curcumin in the bacteria cells. The colonies can be highlighted when both images are superposed, showing the correspondence between the colonies seen by the reflection and fluorescence images.

The confocal reflectance images present the surface of the metal sample. It was expected that the orthodontic appliance squares show some roughness, and appears in the image as a random pattern. It is also noticed a pattern similar to a bunch of grapes, and the superposition of the reflectance and fluorescence images confirm that those patterns correspond to the biofilm. Meanwhile, the fluorescence image show red and yellow-green dots. The yellow-green dots are the fluorescence of the curcumin within the bacteria cells. The red dots are the fluorescence if the propidium iodide (PI) dye, which appear in the dead cells.
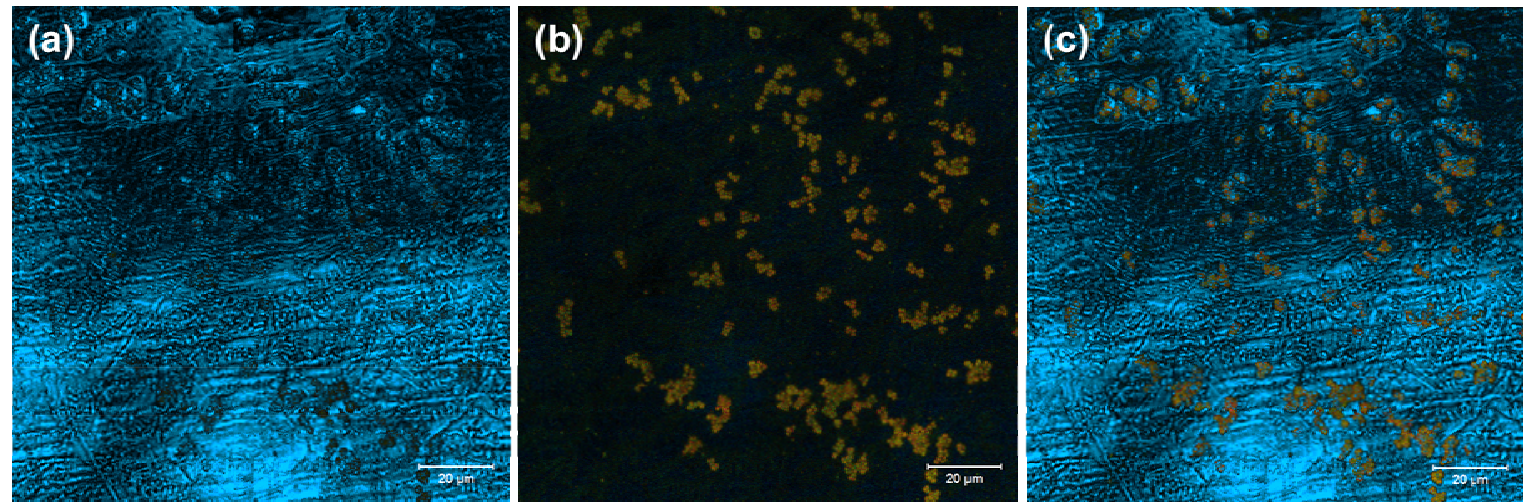

Fig. 2 Biofilms of S. mutans on metallic sample with 7 days, after PDT treatment: (a) Confocal reflection imaging, the image show the appearance of the metal surface, indicating the presence of $S$. mutans colonies; (b) confocal fluorescence image, showing the fluorescence of the propidium iodide (PI) dye and curcumin. The colonies appear as bright spots in the image, red indicates dead cells and yellowish-green show the living microorganisms and (c) reflection and fluorescence images superposed $(a+b)$. The image shows the correspondence among the colonies seen by reflection and fluorescence images. The scale bar corresponds to $20 \mu \mathrm{m}$. 
The bacterial effects of PDT and the surfactant were evaluated by the cell viability (CFU/ml). The efficacy of the treatment differs depending on the association of curcumin and surfactant (Fig. 3). They showed significant reductions in the viability of the $S$. mutans (over $1 \log 10$ ) for all groups, except for the experimental conditions (P-L-S+) and (P+L-S-). The highest reduction in the cell viability of the biofilms was equivalent to $5.6 \log 10(\mathrm{CFU} / \mathrm{ml})$ and correspond to the association of PDT and surfactant.

The results show remarkable behavior of the bacteria population depending on the association of curcumin, surfactant and the application of light (Table 1). In some cases the illumination can promote biostimulation, but none of the studied cases presented a relatively relevant increase on the bacterial population. Applying just PDT $(\mathrm{P}+\mathrm{S}-\mathrm{L}+)$ or surfactant $(\mathrm{P}-\mathrm{S}+\mathrm{L}-)$, it was observed small decreases on the $S$. mutans population. The same occur when light was applied mutually with surfactant (P-S $+\mathrm{L}+$ ), but in this case the antibacterial effect was somehow smaller. The test group where only the photosensitizer was used (P+S-L-) was statistically similar to the control group, confirming that curcumin, by itself, is not capable to a significant bacteria inactivation. On the other hand, the association of curcumin and SDS (P+S+L-) upheld an even smaller decrease in the population. However, when the three where used together $(\mathrm{P}+\mathrm{S}+\mathrm{L}+)$, it was observed a huge antibacterial effect (-5.6 log10). In this case, only one in a thousand part of the population survived.

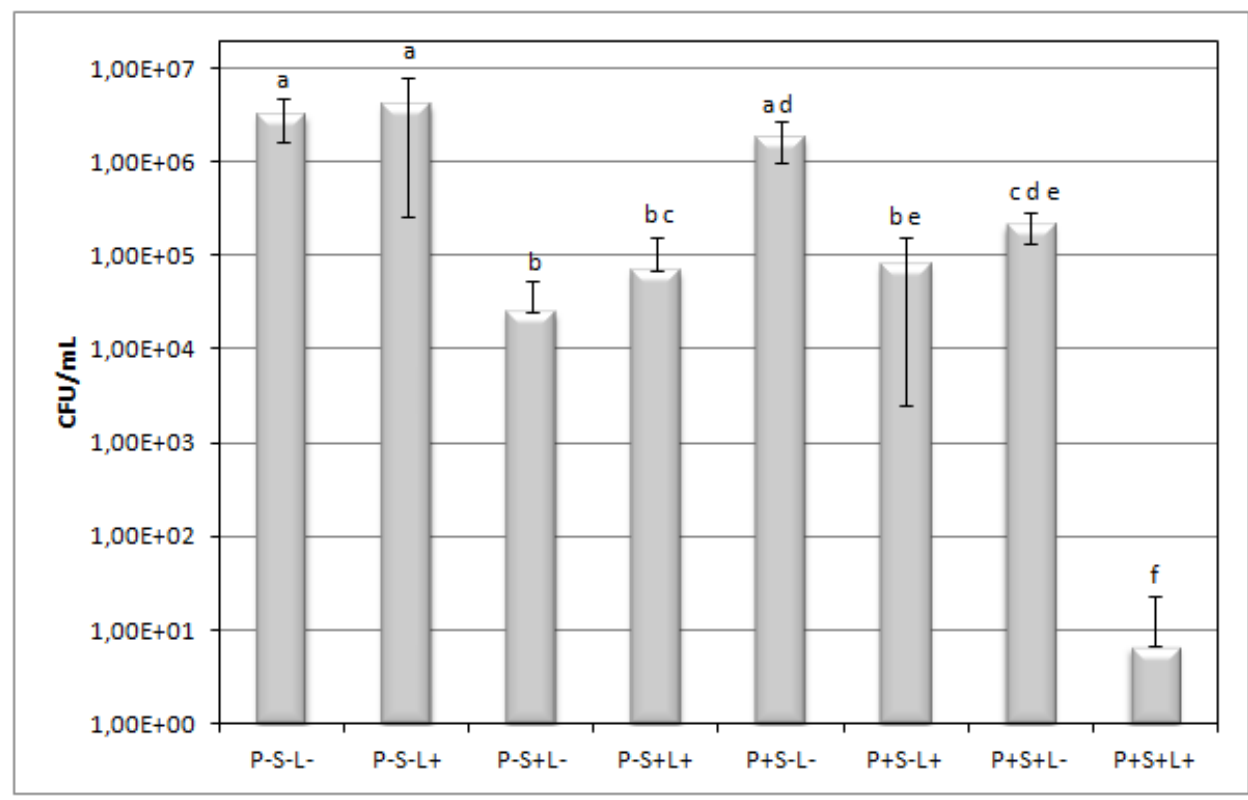

Fig. 3 Reduction of CFU for S. mutans biofilms. Individual difference made by ANOVA One Way: post hoc Turkey. Groups with the same letter are not significantly different $(p>0.005)$.

Table 1 Expression of cell viability $(\mathrm{CFU} / \mathrm{ml})$, related to the control group data, for all experimental groups. Groups with the same letter are not significantly different $(p>0.005)$.

\begin{tabular}{lll}
\hline Experimental groups & CFU percentage difference & Expression $(\log 10)$ \\
\hline (P-S-L-) $^{\text {a }}$ & 0 & \\
$(\mathrm{P}-\mathrm{S}-\mathrm{L}+)^{\text {a }}$ & +29.17 & +0.1 \\
$(\mathrm{P}-\mathrm{S}+\mathrm{L}-)^{\text {b }}$ & -99.22 & -2.1 \\
$(\mathrm{P}-\mathrm{S}+\mathrm{L}+)^{\mathrm{bc}}$ & -97.84 & -1.6 \\
$(\mathrm{P}+\mathrm{S}-\mathrm{L}-)^{\text {a d }}$ & -42.91 & -0.2 \\
$(\mathrm{P}+\mathrm{S}-\mathrm{L}+)^{\text {b e }}$ & -97.46 & -1.5 \\
$(\mathrm{P}+\mathrm{S}+\mathrm{L}-)^{\text {cd e }}$ & -93.37 & -1.1 \\
$(\mathrm{P}+\mathrm{S}+\mathrm{L}+)^{\mathrm{f}}$ & -99.999792 & -5.6 \\
\hline
\end{tabular}


The oral microbiota is composed of more than 500 types of microorganisms, even though $S$. mutans is the etiologic agent of caries and demineralization. It is also responsible for the adhesion, colonization and dental biofilm formation [14]. This study evaluated particularly the susceptibility of $S$. mutans biofilm on the metal surface $(\mathrm{NiCr})$ of orthodontic blade band by means of photodynamic therapy with curcumin (1 $\mathrm{mg} / \mathrm{mL}$ ), $0.1 \%$ of SDS and LED illumination. As expected, we have shown that photodynamic therapy reduces the number of living cells of $S$. mutans in a biofilm model created on metallic surface [15-17]. The presence of photosensitizer, without irradiation, was not able to achieve an effective antibacterial outcome. This confirms the essential rule of light to obtain an anti-microbial effect $[18,19]$ by means of PDT.

The most significant finding was the lack of viability of $S$. mutans on the surfaces of the samples treated with curcumin combining PDT with surfactant. The result for this group $(\mathrm{P}+\mathrm{S}+\mathrm{L}+)$ is above the minimal bactericidal concentration, which is the minimum concentration able to reduce $4 \log 10$ on the survival of a microorganism [20]. Significant decrease on the viability of microorganisms was also observed when biofilms were exposed to the surfactant alone (P-S+L-), surfactant with light $(\mathrm{P}-\mathrm{S}+\mathrm{L}+)$, photosensitizer with light $(\mathrm{P}+\mathrm{S}-\mathrm{L}+)$, or the combined use of surfactant and curcumim without light $(\mathrm{P}+\mathrm{S}+\mathrm{L}-)$, compared to the control group (P-S-L-). For these groups, the reduction on the bacteria viability was above $90 \%$. Depending on the application, $90 \%$ of reduction on a microorganism population might be sufficient. Although the results show that the bacterial inactivation can be almost complete when the surfactant was used together with PDT.

The surfactants are amphipathic molecules composed of a hydrophobic portion and a hydrophilic portion. The first is often a nonpolar hydrocarbon chain, while the second can be ionic (cationic or anionic), nonionic or amphoteric. The surfactants, for their biochemical characteristics, are highly potent and act as emulsifiers, reducing interfacial and superficial tension [21]. They are responsible for modifications on the ion channels of the bacterial membrane via trans-membranes proteins, similar to the porins. This allows the passage of solutes through the membrane, increasing the permeability of these membranes [21-24]. Surfactants also work as: emulsifiers, dispersing in water hydrocarbons or other insoluble compounds; reducing the adhesion and releasing surfaces cell; and presenting antibiotic activity.

The mechanism of action of PDT is not connected to the mediation of chemical radicals acting in a single target, which is the case of the antibacterial products. Photosensitizes act producing reactive oxygen species (ROS) which have no specific site of action. This avoids the formation of strains resistant to antibiotics [20, 21, 25, 26]. Photodynamic therapy applied as antimicrobial therapy can be also advantageous over mechanical removal methods, since it can reach places such as recesses and protrusions of the orthodontic accessories [27]. It may also enable safe treatment of patients with special needs or difficulty in oral hygiene during orthodontic treatment, avoiding oral infections, such as dental caries, gingivitis and periodontal pockets [13, 27].

Several hypotheses could explain the synergism of the PDT action with surfactant as antimicrobial therapy in $S$. mutans biofilm. The surfactant could be increasing the number of working cycles of PS, protecting it from oxidation, and increasing the efficacy of PDT. It could also be acting increasing the permeability of the curcumin through the cell membrane, increasing the concentration of PS molecules in the bacteria. One other hypothesis is that the surfactant is breaking the biofilm structure, and this allows the PDT to be more effective. Although the surfactant, SDS, can be used as antimicrobial by itself, the combined effects of it with PDT cannot explain such efficacy of the treatment. The reduction on bacteria viability was larger than one could expect from using both treatments separately. Therefore more 
studies must be done in order to thoroughly understand this mechanism.

\section{Conclusions}

The result of this study shows higher reduction in the S. mutans population, compared to previous investigations [15, 19]. We demonstrate significant removal of $S$. mutans biofilm after application of PDT with SDS as antimicrobial therapy. PDT associated to surfactants promoted a high inactivation of $S$. mutans biofilm (5.6log10) on the surface of metallic orthodontic accessories. We have shown that, even though the biofilm is very tough and complex structure, we are able to promote almost the complete inactivation of $S$. mutans in a system similar to an orthodontic treated patient's mouth. And this inactivation was achieved with a protocol that does not promote bacterial resistance that applied PDT and SDS.

Given the findings, our results suggest that the surfactant acts on the microorganisms' membrane making them permeable to solutes, and breaking the biofilm structure. The surfactant leads to higher the input of oxygen molecules and curcumin from the extracellular medium into the microorganism. Therefore, the synergistic action of the SDS and PS produces higher amounts of reactive oxygen species (ROS) upon irradiation, optimizing the antimicrobial effect of PDT. At the same time, protecting the PS molecules from oxidation and increasing its lifetime of the PDT cycle. These hypotheses should be put to test in supplementary investigations.

\section{Aknowledgments}

The authors would like to acknowledge the support of the Brazilian agencies FAPESP, CNPQ and CAPES, through founding and scholarships. V. H. Panhóca would like to acknowledge scientific contributions and helpful advice from Clóvis Grecco and Fernanda Rossi Paolillo.

\section{References}

[1] P.H. Keyes, The infectious and transmissible nature of experimental dental caries, Findings and implications, Arch. Oral. Biol. 1 (1960) 304-320.

[2] E. Newbrum, Cariology, 2nd ed., Willians na Wilkins, Baltimore, 1978.

[3] T. Fusayama, S. Terachima, Differentiation of two layers of carious dentin by staining, J. Dent. Res. 51 (3) (1972) 866.

[4] T. Fusayama, S. Terashima, Differentiation of two layers of carious dentin by staining, Bull Tokyo Med. Dent. Univ. 19 (1) (1972) 83-92.

[5] M.J. Sedlacek, C. Walker, Antibiotic resistance in an in vitro subgingival biofilm model, Oral. Microbiol. Immunol. 22 (5) (2007) 333-339.

[6] C.R. Fontana, A.D. Abernethy, S. Som, K. Ruggiero, S. Doucette, R.C. Marcantonio, et al., The antibacterial effect of photodynamic therapy in dental plaque-derived biofilms, J. Periodontal Res. 44 (6) (2009) 751-759.

[7] J.A. Corbett, L.R. Brown, H.J. Keene, I.M. Horton, Comparison of Streptococcus mutans concentrations in non-banded and banded orthodontic patients, J. Dent. Res. 60 (12) (1981) 1936-1942.

[8] S. George, A. Kishen, Photophysical, photochemical, and photobiological characterization of methylene blue formulations for light-activated root canal disinfection, Journal of Biomedical Optics 12 (3) (2007) 034029.

[9] R. Darlenski, J.W. Fluhr, Photodynamic therapy in dermatology: past, present, and future, Journal of Biomedical Optics 18 (6) (2012) 061208.

[10] M. Wainwright, Photodynamic antimicrobial chemotherapy (PACT), Journal of Antimicrobial Chemotherapy 42 (1) (1998) 13-28.

[11] H. Greenwell, N. Bissada, Emerging concepts in periodontal therapy, Drugs 62 (18) (2002) 2581-2587.

[12] P. Bidault, F. Chandad, D. Grenier, Risk of bacterial resistance associated with systemic antibiotic therapy in periodontology, Journal of the Canadian Dental Association 73 (8) (2007) 721-725.

[13] N. Araujo, C. Fontana, M. Gerbi, V. Bagnato, Overall-mouth disinfection by photodynamic therapy using curcumin, Photomedicine and Laser Surgery 30 (2) (2012) 96-101.

[14] J. Kreth, E. Hagerman, K. Tam, J. Merritt, D.T. Wong, B.M. Wu, et al., Quantitative analyses of Streptococcus mutans biofilms with quartz crystal microbalance, microjet impingement and confocal microscopy, Biofilms 1 (4) (2004) 277-284.

[15] Z. Zou, P. Gao, H. Yin, Y. Li, Investigation of photodynamic therapy on streptococcus mutans of oral biofilm, Chinese Optics Letters 6 (12) (2008) 947-949.

[16] J. Giusti, L. Santos-Pinto, A. Pizzolito, K. Helmerson, E. Carvalho-Filho, C. Kurachi, et al., Antimicrobial photodynamic action on dentin using a light-emitting 
diode light source, Photomedicine and Laser Surgery 26 (4) (2008) 281-287.

[17] I.C. Zanin, M.M. Lobo, L.K. Rodrigues, L.A. Pimenta, J.F. Höfling, R.B. Gonçalves, Photosensitization of in vitro biofilms by toluidine blue $\mathrm{O}$ combined with a light-emitting diode, Eur. J. Oral. Sci. 114 (1) (2006) 64-69.

[18] J. O'Neill, C. Hope, M. Wilson, Oral bacteria in multi-species biofilms can be killed by red light in the presence of toluidine blue, Lasers in Surgery and Medicine 31 (2) (2002) 86-90.

[19] M. Schneider, G. Kirfel, M. Berthold, M. Frentzen, F. Krause, A. Braun, The impact of antimicrobial photodynamic therapy in an artificial biofilm model, Lasers in Medical Science 27 (3) (2012) 615-620.

[20] G. Jori, C. Fabris, M. Soncin, S. Ferro, O. Coppellotti, D. Dei, et al., Photodynamic therapy in the treatment of microbial infections: Basic principles and perspective applications, Lasers Surg. Med. 38 (5) (2006) 468-481.

[21] P.A.V. Fernandes, I.R.d. Arruda, A.F.A.B.d. Santos, A.A.d. Araújo, A.M.S. Maior, E.A. Ximenes, Antimicrobial activity of surfactants produced by Bacillus subtilis R14 against multidrug-resistant bacteria, Brazilian Journal of Microbiology 38 (2007) 704-709.
[22] F. Peypoux, J.M. Bonmatin, J. Wallach, Recent trends in the biochemistry of surfactin, Appl. Microbiol. Biotechnol. 51 (5) (1999) 553-563.

[23] M. Nitschke, G.M. Pastore, Biosurfactants: Properties and applications, Chemicals New 25 (2002) 772-776.

[24] J.P. Lyon, R.R. Rezende, M.P. Rabelo, C.J. de Lima, L.M. Moreira, Synergic effect of photodynamic therapy with methylene blue and surfactants in the inhibition of Candida albicans, Mycopathologia 175 (1-2) (2013) 159-164.

[25] F. Lauro, P. Pretto, L. Covolo, G. Jori, G. Bertoloni, Photoinactivation of bacterial strains involved in periodontal diseases sensitized by porphycene-polylysine conjugates, Photochemical \& Photobiological Sciences 1 (7) (2002) 468-470.

[26] A. Tavares, C.M. Carvalho, M.A. Faustino, M.G. Neves, J.P. Tomé, A.C. Tomé, et al., Antimicrobial photodynamic therapy: Study of bacterial recovery viability and potential development of resistance after treatment, Mar Drugs 8 (1) (2010) 91-105.

[27] T.S. Mang, D.P. Tayal, R. Baier, Photodynamic therapy as an alternative treatment for disinfection of bacteria in oral biofilms, Lasers Surg. Med. 44 (7) (2012) 588-596. 


\title{
Efficiency Improvement of $590 \mathrm{~nm}$ AIGalnP Light Emitting Diode with a Reflective Top Electrode
}

\author{
Hyung Joo Lee ${ }^{1}$, Young Dae Cho ${ }^{1}$, Young Jin Kim ${ }^{1}$, Choong Hun Lee ${ }^{2}$, Jae Hoon Kim ${ }^{2}$, Hwa Sub $\mathrm{Oh}^{3}$ and $\mathrm{Su}$ \\ Chang $\mathrm{Ahn}^{3}$ \\ 1. CF Technology Division, AUK Corporation, Iksan 570-210, Republic of Korea \\ 2. Division of Microelectronics and Display Technology, Wonkwang University, Iksan 570-749, Republic of Korea \\ 3. LED Team, Korean Photonic Technology Institute (KOPTI), Gwangju 500-779, Republic of Korea
}

Received: November 08, 2013 / Accepted: December 17, 2013 / Published: February 15, 2014.

\begin{abstract}
In this work, enhancement of the light extraction efficiency of a $590 \mathrm{~nm}$ AlGaInP light-emitting diodes (LED) with a reflective top electrode (RTE) was investigated. A distributed Bragg reflector (DBR), consisting of AlAs/AlGaAs pairs, grown on an $\mathrm{AlGaInP}$ structure was used as a reflector for a reflective top electrode. It was found that a higher output power was observed from the AlGaInP LED with a RTE than from a conventional one. In addition, it was noted that the improvement in the output power depends strongly on the reflectivity of the reflector and that it exhibits a more effective performance with low injection currents. The increase in the optical output power was attributed to the enhanced extraction efficiency caused by a reduction of light absorbed from the emission region to top electrode through the RTE.
\end{abstract}

Key words: AlGaInP, reflective top electrode (RTE), light-emitting diode (LED).

\section{Introduction}

III-V compound semiconductors are known as an attractive material for optical devices such as solar cells [1], laser diodes [2], and light-emitting diodes [3] amongst others. In particular, light-emitting diodes based on AlGaInP materials, with a wavelength ranging from $570 \mathrm{~nm}$ to $630 \mathrm{~nm}$, have been developed for commercial applications such as illumination, traffic lights, vehicle lighting and indicators [4]. Ever higher efficiency AlGaInP LEDs have been required for high power devices with a low operating voltage. The efficiency of AlGaInP LEDs has been significantly improved by either increasing the internal quantum efficiency by inserting multiple hetero-structures and multiple quantum barriers, or by increasing the light extraction efficiency by using a distributed Bragg

Corresponding author: Hyung-Joo Lee, Ph.D., research fields: light emitting diode, AlGaInP and AlGaAs. E-mail: lee-hyungjoo@auk.co.kr. reflector [5-7], surface texturing [8], bottom window [9] or nanoscale materials deposited on their surfaces [10].

Although numerous studies have been reported for AlGaInP LEDs with a high efficiency, certain intrinsic limitations still exist that considerably decrease their overall efficiency. In particular, it was shown that a significant reduction in the efficiency depends strongly on an opaque top electrode $[11,12]$, as the light-emitting region in the active region is generated mostly beneath the opaque top electrode.

In this study, the fabrication and effects of a reflective top electrode on the light extraction efficiency of a $590 \mathrm{~nm}$ AlGaInP LED were investigated. This reflective top electrode was fabricated by inserting a AlAs/AlGaAs distributed Bragg reflector between the top electrode and the LED structure using a MOVD system. It was found that the light extraction efficiency of $590 \mathrm{~nm}$ AlGaInP LEDs 
could be increased by using the reflective top electrode developed.

\section{Experiments}

The AlGaInP LEDs used were grown on an n-type GaAs (111) substrate at a $10^{\circ}$ forward tilt [0-1-1] \pm $0.5^{\circ}$ using an MOCVD system. Here, trimethylgallium (TMGa), trimethylaluminum (TMAl), and trimethylindium (TMIn) were used as the group III sources; arsine $\left(\mathrm{AsH}_{3}\right)$ and phosphine $\left(\mathrm{PH}_{3}\right)$ were the group $\mathrm{V}$ sources; and disillane $\left(\mathrm{Si}_{2} \mathrm{H}_{6}\right)$ gas and cyclo-peantadienylmagnesium $\left(\mathrm{Cp}_{2} \mathrm{Mg}\right)$ were the $\mathrm{n}$ and p-doping sources. Hydrogen $\left(\mathrm{H}_{2}\right)$ was used as the carrier gas for all sources.

The cross-sectional schematics of the AlGaInP LED structures developed are shown in Fig. 1. In brief, the substrate is a GaAs lattice matched with both AlGaInP and AlGaAs. The AlAs/AlGaAs distribution Bragg reflector (DBR) is used to reflect upward the light emitted from the active region to the substrate. The nand p-AlGaInP materials were used as the n- and p-confinement layers, respectively, by varying the component ratio of $\mathrm{Al}$ and $\mathrm{Ga}$ in the $\mathrm{AlGaInP}$ materials. The active region consists of 30 pairs of MQWs, having $10 \mathrm{~nm}$ thick AlGaInP wells and $30 \mathrm{~nm}$ thick AlGaInP barriers. In conventional AlGaInP LEDs, the GaAs top layer of $80 \mathrm{~nm}$ thick was grown on a pconfinement layer for ohmic contact. In the developed AlGaInP LEDs with a reflective top electrode, the AlAs/AlGaAs top distributed Bragg reflector (TDBR), consisting of $\sim 68 \mathrm{~nm}$ thick AlAs and $\sim 57 \mathrm{~nm}$
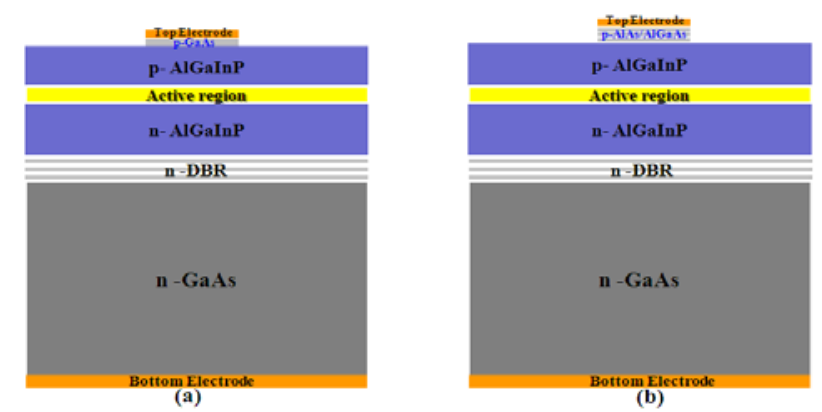

Fig. 1 Schematics of structures of (a) conventional AIGaInP LED and (b) AIGaInP LEDs with a reflective top electrode. thick AlGaAs periods, was grown on the pconfinement layer for both reflection and ohmic contact. For further improved information, the TDBRs of 4 and 12 periods were trialed for the reflective top electrode.

In this work, all the epitaxial wafers used were divided into 9 mil chips $(230 \mu \mathrm{m} \times 230 \mu \mathrm{m})$ using dicing system.

\section{Results and Discussion}

Fig. 2 presents the photoluminescence (PL) measurements for the AlGaInP LED epitaxial wafers with difference structures, which were obtained using a $525 \mathrm{~nm}$ laser diode source, showing that all of these epitaxial wafers have the same wavelength of $590 \mathrm{~nm}$. The figure shows that the intensity of the AlGaInP LEDs was decreased remarkably by the insertion of the TDBR, with the highest intensity being obtained in the AlGaInP LED epitaxial wafer without a TDBR. Additionally, a significant reduction in the intensity close to zero was observed from the AlGaInP LED with the TDBR of 12 periods. From these results, it was confirmed that the light emitted upward from the LED was reflected effectively through the use of a TDBR.

A schematic of the structure of an AlGaInP LED with a reflective top electrode is shown in Fig. 3a. From the schematic, it can be assumed that the emission region in the active layerwas generated

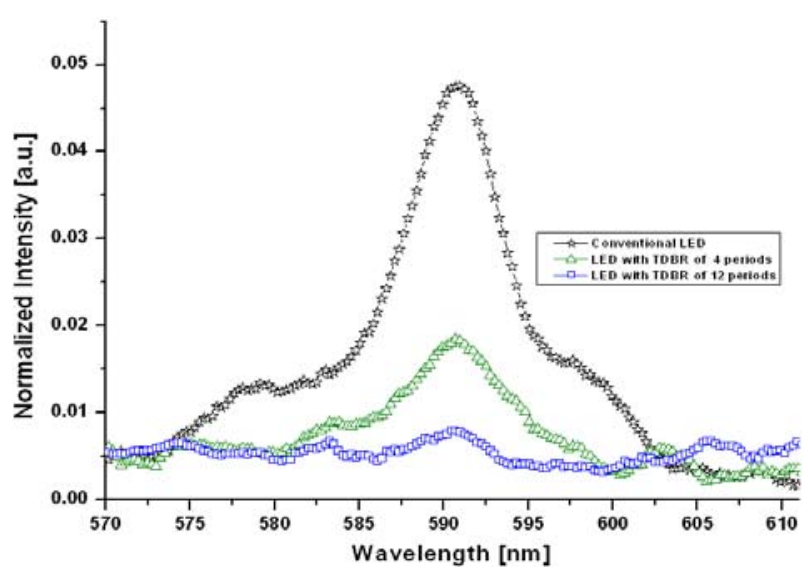

Fig. 2 Photo-luminescence measurement of AIGaInP LED epi-wafer with different structures. 

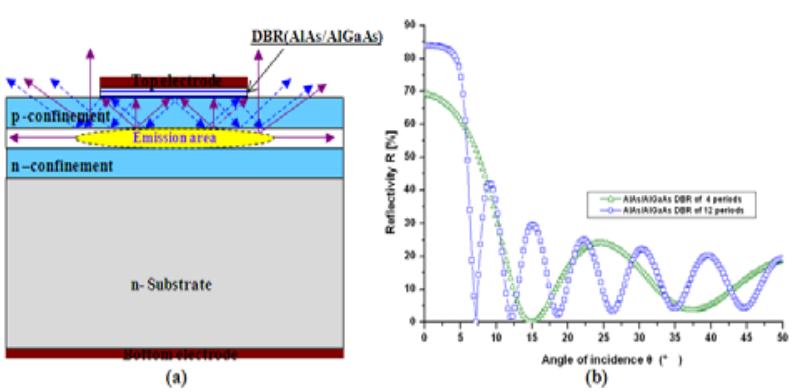

Fig. 3 (a) Light emitting path on lights emitted upwardly from emission area in AIGaInP LED chip with the RTE and (b) graphic of reflectivity and angle of incidence for the different DBRs used as reflector.

mainly below the top electrode, and that most of the light emitted upward from the emission region was moved to the top electrode. Therefore, the efficiency of a conventional LED must be low due to the top electrode being absolutely opaque to the light injected. On the other hand, an even higher efficiency could be obtained from an AlGaInP LED with a reflective top electrode because light emitted from the emission area to the absorbing top electrode was reflected by a reflective top electrode. It seems to be resonant cavity effect caused by reflective top electrode. Also, it was known that light extraction efficiency of LED was increased by resonant cavity effect caused

Fig. $3 b$ shows the angle of incidence $(\theta)$ and reflectivity (R) of an AlAs/AlGaAs DBR, which is used as the reflector for the reflective top electrode, for the different periods. In Fig. $3 \mathrm{~b}, \sim 70 \% \mathrm{R}$ and $15^{\circ} \theta$ were observed at the DBR of 4 periods. The reflectivity and angle of incidence were increased or decreased respectively, with increasing periods of the DBR because they have trade-off relationship [4]. At 12 periods, $\sim 70 \% \mathrm{R}$ and $7.5^{\circ} \theta$ were observed.

For further improved performance, the light-current-voltage (L-I-V) characteristics of $590 \mathrm{~nm}$ AlGaInP LED chips with different structures were measured under an injection current ranging from 0 $\mathrm{mA}$ to $40 \mathrm{~mA}$ as shown in Fig.4. In the current-voltage (I-V) characteristics, all of the samples show nearly the same operating voltages. The operating voltage of the LED chips developed was barely influenced by the presence and thickness of the TDBR because the AlAs

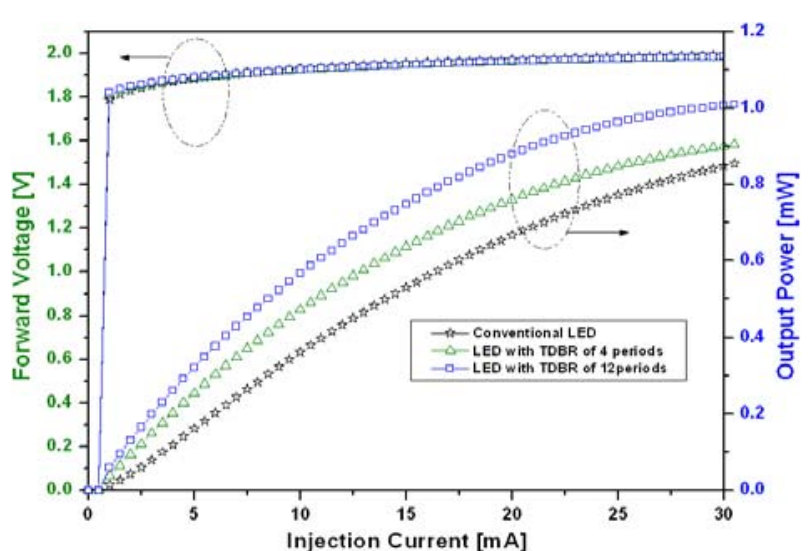

Fig. 4 (a) Comparison of the $L-I-V$ characteristic curves of the conventional AIGaInP LED chip and AIGaInP LED chip with RTE having the TDBR of various periods.

and $\mathrm{AlGaAs}$ materials used for the reflector have much lower resistivity than the underlying structures [13].

Distinct results were observed in the $L-I$ characteristics of the $590 \mathrm{~nm}$ AlGaInP LED chips with different structures. Clearly the LED chips with a reflective top electrode show a higher output power than the conventional LED chips. Also, it was found that the higher output power was obtained at DBR of 12 periods. At a high injection current of $30 \mathrm{~mA}$, output power of $1 \mathrm{~mW}$ obtained for a DBR of 12 periods shows relative increase of $23 \%$ and $18 \%$ respectively, as compared to without and with 4 periods. The remarkable difference of output power between LEDs without and with 4 periods was not observed. Therefore, this results presented that cavity effect caused by insertion of RTE was depends strongly on reflectivity of DBR used.

\section{Conclusions}

In this work, efficiency improvements for a $590 \mathrm{~nm}$ AlGaInP light-emitting diode (LED) with a reflective top electrode (RTE) were investigated. A distributed Bragg reflector (DBR), consisting of $\mathrm{AlAs} / \mathrm{AlGaAs}$ pairs, was used as a reflector for a reflective top electrode. It was found that a higher output power was observed from the AlGaInP LED with a RTE as compared to a conventional one. The output power of the LEDs with a RTE depends strongly on the reflectivity of the DBR used as its reflector. At an 
injection current of $30 \mathrm{~mA}$, the highest output power of $1 \mathrm{~mW}$ was obtained from a $590 \mathrm{~nm}$ AlGaInP LED having the 12 pairs DBR with $\sim 85 \% \mathrm{R}$, which showed a relative increase of around $23 \%$ over a conventional LED.

\section{Acknowledgments}

The authors wish to express their appreciation to the Human Resources Development program (No. 20134030200250) of the Korea Institute of Energy Technology Evaluation and Planning (KETEP) grant funded by the Korea government Ministry of Trade, Industry and Energy, and by a Korea Research Foundation Grant from the ATC project (No. 10035863 ) by the Ministry of Knowledge Economy

\section{References}

[1] X.Q. Liu, C.M. Fetzer, E.Rehder, H. Cotal, S. Mesropian, D. Law, et al., Organometallic vapor phase epitaxy growth of upright metamorphic multi-junction solar cells. Journal of Crystal Growth 352 (2012) 186-189.

[2] T. Onishi, K. Inoue, K.O. Onozawa, T. Takayama, M. Yuri, High power and high temperature operation of $\mathrm{Mg}$ doped AlGaInP based red laser diodes, IEEE Journal of Quantum Electronics 40 (2004) 1634-1638.

[3] H.J. Lee, S.U. Kim, J.H. Kim, Effects of rough p-GaN layer on improving light extraction efficiency of $630-\mathrm{nm}$ AlGaInP LEDs, IEEE Electron Devices Letters 34 (2013) 906-908.

[4] E.F. Schubert, Light Emitting Diodes Cambridge University, London, 2006, Ch.1, Ch. 10.

[5] H.S. Oh, S.M. Kim, J.H. Joo, J.H. Baek, J.S. Kwak, Optimization of the number of quantum well pairs for high brightness AlGaInP based light emitting diodes, Journal of Nanoscience and Nanotechnology 11 (2011) 1503-1506.

[6] C.S. Chang, Y.K. Su, S.J. Chang, P.T. Chang, Y.R. Wu, K.H. Huang, et al., High-bringthness AlGaInP 573-nm light emitting diode with a chirped multiquantum barrier, IEEE Journal of Quantum Electronics 34 (1998) 77-83.

[7] R. RoBbach, W.M. Schulz, M. Reischle, G.J. Beirne, M. Jetter, P. Michler, Increased single-photon emission from $\mathrm{InP} / \mathrm{AlGaInP}$ quantum dots grown on $\mathrm{AlGaAs}$ distributed Bragg reflectors, Journal of Crystal Growth 3 (2008) 4818-4820.

[8] L.J. Yan, J.K. Sheu, W.C. Wen, T.F. Liao, M.J. Tsai, C.S. Chang, Improved light extraction efficiency in AlGaInP light emitting diodes by applying a periodic texture on the surface, IEEE Photonic Technology Letter 20 (2008) 1724-1726.

[9] H.J. Lee, Y.J. Kim, S.U. Kim, J.U. Jo, C.H. Lee, J.H. Kim, et al., Efficiency improvement of $630 \mathrm{~nm}$ AlGaInP light-emitting diodes based on AlGaAs bottom window, Japaness Journal of Applied Physics 52 (2013) 1021011-1021014.

[10] H.J. Lee, E.J.D. Castro, J.H. Kim, C.H. Lee, Effects of nanosized $\mathrm{Ni}$ particle structure on the enhancement of light extraction from $600 \mathrm{~nm}$ AlGaInP light emitting diodes, Applied Physics Express 5 (2012) 1221021-1221023.

[11] R.M. Flectcher, C.P. Kuo, T.D. Osentowski, K.H. Huang, M.G. Craford, The growth and properties of high performance AlGaInP emitter using a lattice mismatch $\mathrm{GaP}$ window layer, Journal of Electronics Materials 20 (1991) 1125-1130.

[12] H. Sugawara, K. Itaya, H. Nozaki, G. Hatakoshi, High-brightness InGaAlP green light-emitting diodes, Applied Physics Letters 61 (1992) 1775-1777.

[13] H. Sugawara, M. Ishikawa, G. Hatakoshi, High efficiency InGaAlP/GaAs visible light-emitting diodes, Applied Physics Letters 58 (1991) 1010-1012. 


\title{
Compensation Method: New Perspectives
}

\author{
Boris Levin \\ Holon Institute of Technology, Holon 58102, Israel
}

Received: July 31, 2013 / Accepted: September 20, 2013 / Published: February 15, 2014.

\begin{abstract}
The compensation method aiming to reduce the irradiation on the user's body and to form a weak field area (a dark spot) in an antenna near region is considered. The results of the method development for the use in a narrow frequencies range are given. They show that under realistic conditions the compensation method allows reducing the irradiation power by a factor of 3-4 and more. The pattern changes are predictable and small, if the points of antennas placement are selected correctly. The dark spot necessity near the transmitter is not limited by mobile communication. A man often uses a low-power radio station, which is placed nearby in a vehicle, and it is necessary to protect from irradiation not only user but also other passengers. In the paper, it is shown that broadband field compensation can be achieved in various structures where the needed anti-phase fields are created either by identical antennas or by radiators located at equal distance from the compensation point, or by flat reflectors.
\end{abstract}

Key words: Antenna theory, compensation, mobile antennas, mutual coupling, near fields.

\section{Introduction}

The compensation method was proposed to protect user's head against strong electromagnetic fields of a cellular phone antenna. The method was intended to decide the particular problem of decreasing near region field created with the antenna of the certain device operated at the one given frequency. A cellular phone is, in particular, such a device. During a phone conversation, it is placed next to user's head and irradiates sensitive human organs (brain, eye, etc.). The absorbed energy in today's cellular phones can be as high as a half of all radiated energy.

But the problem is not limited to the cellular phones, since, firstly, a man often uses or services a low-power transmitter, which he carries or places nearby in a production area or in a vehicle. Secondly, one must protect against a strong electromagnetic radiation not only living organisms, but also devices. The protection of devices is necessary, since the irradiation of nearby devices can disrupt their normal operation, cause

Corresponding author: Boris Levin, doctor, research fields: electromagnetic theory, theory of linear antennas and antennas optimization and electromagnetic compatibility. E-mail: levinpaker@gmail.com. spontaneous device switching on and switching off, change operating regimes, etc..

The rather obvious idea of head protection by means of screening, i.e., by shading effect, is not practicable. The near field has no ray structure, and hence the shadow formed behind a metal screen can only cover an area approximately equal to the screen size. For example, in order to protect the head of the cellular phone user, the screen must be much larger than the cross section of the handset housing. For similar reasons, one should discard the idea of using an absorber, i.e., a dielectric shield that absorbs some part of energy. The distortion of the antenna pattern is another obvious disadvantage of using screens and absorbers.

The protective action of the compensation method proposed by Bank [1] is based on another principle: the mutual suppression of fields created by various radiating elements in a certain area. The diverse variants of the principle implementation (interference of two linear radiators fields, folded antenna, and cavity antenna [2, 3]) possess a potentiality to reduce irradiation of user's organism, especially his head, 
without distorting the antenna pattern in the horizontal plane.

In accordance with the key variant of the compensation method, as shown in Fig. 1, main radiator 1 is supplemented with second (auxiliary) radiator 2 situated in the plane passing through the head center and the feed point of the main radiator. The second radiator is placed between the head and the main radiator and is excited approximately in anti-phase to it (not exactly in anti-phase because the phase of the field progresses along the interval between the radiators). So, the radiator fields will compensate each other at a certain point inside the head, and the point will be surrounded with a zone of a weak field: a dark spot will arise.

In order to obtain the same field strengths at the compensation point, when the radiators driving currents differ substantially, it is enough to separate the radiators by a small fraction of the wavelength, e.g. a distance of about $1-2 \mathrm{~cm}$ at $1 \mathrm{GHz}$. The relatively small driving current of the auxiliary radiator ensures that upon appropriate placement of the radiators the total field at far region is almost unaffected by the presence of the auxiliary radiator, and the pattern remains omnidirectional, allowing to decide the crucial question in the development of any method of reducing exposure, how to preserve the communication performances of the system.

Results of key variant analysis are described in Section 2.

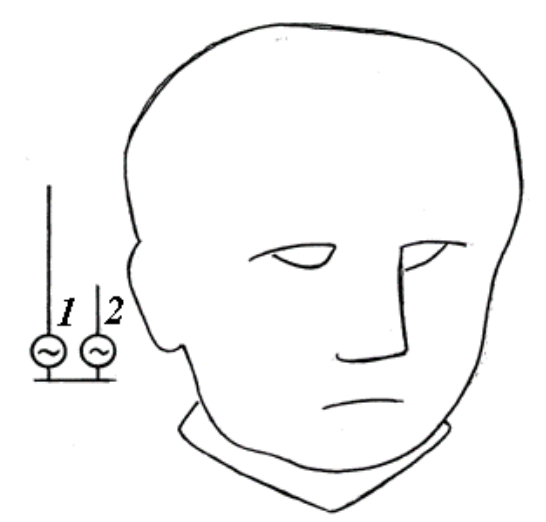

Fig. 1 Two radiators next to the head.

\section{Compensation at One Frequency}

The problem of field calculation in the space surrounding a radiator is complicated due to the heterogeneous nature of this medium. Cellular handset antennas are in close proximity to the user's head, hand and body, which contain multiple tissues with different permittivity, much higher than that of the free space. If an antenna is tangent to the user's head and is considered in a crude approximation as a linear radiator located along the (plane) boundary between two half-spaces: air with $\varepsilon_{r}=1$ and a head with $\varepsilon_{r} \neq 1$, then an analogy is valid for a quasi-stationary electrical field (including an antenna field in the near region) created by alternating linear current and an electrostatic field of charged conducting bodies. On the other hand, it is known that an electrostatic field of an isolated wire located at the boundary of two media coincides with its field in a homogeneous medium with a permittivity equal to the average of both media ones [4].

This analogy allows reducing the calculation of an antenna near field in a piecewise-homogenous medium to approximate field calculation in a homogenous medium. But the near field of an antenna has a quasi-stationary character only in the first order approximation. The radiators are located near the head, but not tangent to a head. An assumption that radiators are located along on interface of two half-spaces is too crude. Therefore, the results are in need of check-up with the help of calculation program, for example CST.

The circuit of radiators displacement is given in Fig. 2. Here, $A_{1}$ and $A_{2}$ are the main and the auxiliary

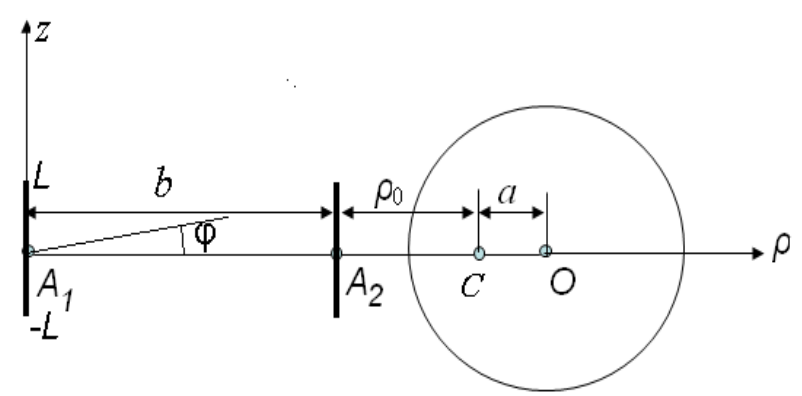

Fig. 2 The diagram of phone radiator placement close to a human head. 
radiator, $C$ is the compensation point, $O$ is the head center. The radiators are the symmetrical vertical electric dipoles with finite length. The field components of the main radiator $A_{1}$ in cylindrical coordinates $(\rho, \varphi, z)$ are given by:

$$
\begin{aligned}
& E_{z 1}=-j \frac{30 J_{A 1}}{\varepsilon_{r} \sin k L_{1}}\left[\exp \left(-j k R_{1}\right) / R_{1}+\right. \\
& \left.+\exp \left(-j k R_{2}\right) / R_{2}-2 \cos k L_{1} \exp \left(-j k R_{0}\right) / R_{0}\right], \\
& E_{\rho 1}=j \frac{30 J_{A 1}}{\varepsilon_{r} \rho_{1} \sin k L_{1}}\left[\left(z-L_{1}\right) \exp \left(-j k R_{1}\right) / R_{1}+\right. \\
& \left.+\left(z+L_{1}\right) \exp \left(-j k R_{2}\right) / R_{2}-2 z \cos k L_{1} \exp \left(-j k R_{0}\right) / R_{0}\right]
\end{aligned}
$$

here, $J_{A 1}$ is the driving current, $L_{1}$ is the dipole arm length, $R_{1}=\sqrt{\left(z-L_{1}\right)^{2}+\rho_{1}^{2}}$ is the distance from the observation point $M$ to the radiator's top edge, $R_{2}=\sqrt{\left(z+L_{1}\right)^{2}+\rho_{1}^{2}}$ is the distance from $M$ to the radiator's bottom edge, $R_{0}=\sqrt{z^{2}+\rho_{1}^{2}}$ is the distance from $M$ to the radiator center, and $\rho_{1}=\rho$. For the auxiliary radiator $A_{2}$ one can write analogous expressions with $L=L_{2}, \rho_{2}{ }^{2}=\rho_{1}{ }^{2}+b_{2}-2 \rho_{1} b \cos \varphi \rho_{2}{ }^{2}$, where, $b$ is the distance between antennas. The field components of a monopole of the same arm length are smaller by factor 2 .

At the compensation point $\mathrm{C}$, the both radiators fields must have the same magnitudes and opposite signs. But (1) shows that, in general, simultaneous cancellation of $E_{z}$ and $E_{\rho}$ by adjustment of the current $J_{A 2}$ is impossible. Since, $E_{z}$ is greater than $E_{\rho}$, and $E_{\rho}=$ 0 along the $\rho$-axis (at $z=0$ ), we choose to cancel the $E_{z}$ components:

$$
E_{z 2}\left(b+\rho_{0}, 0,0\right)=-E_{z 1}\left(b+\rho_{0}, 0,0\right)
$$

here, $\rho_{0}$ is the distance from antenna $A_{2}$ to the compensation point.

For the sake of simplicity, we assume that the dipoles have equal dimensions. The case of dipoles with different lengths is studied in Ref. [5].

The mutual coupling between closely located antennas results in some variations in their characteristics. Find the current and the input impedance of two adjacent finite length monopoles, located within the near region of each other [6]. In accordance with the theory of folded radiator and the superposition principle, one can analyze the two-radiator system with the generator exciting one of the wires as a superposition of two sub-systems: an even-mode sub-system (with in-phase currents), and an odd-mode sub-system (with anti-phased currents). The terminal currents $J_{A 1}$ and $J_{A 2}$ of the radiators excited by the generators are $e_{1}$ and $e_{2}$ are

$$
\begin{aligned}
J_{A 1} & =\left(e_{1}-e_{2}\right) Y_{1}+\left(e_{1}+e_{2}\right) Y_{2} \\
J_{A 2} & =\left(e_{2}-e_{1}\right) Y_{1}+\left(e_{1}+e_{2}\right) Y_{2}
\end{aligned}
$$

where, $Y_{1}=1 /\left[\left(-j W_{1} \cot k L+2 R\right)\right]$ is the input admittance of the long line formed with two monopoles, $Y_{2}=1 /\left[\left(4 Z_{m}\left(L, a_{e}\right)+2 R\right)\right]$ is the input admittance depending on the admittance $Z_{m}\left(L, a_{e}\right)$ of the monopole of the length $L$ and radius $a_{e}=\sqrt{a b}, W_{l}=120 \ln (b / a)$ is the wave impedances of the line, $k=2 \pi / \lambda$ is the propagation constant, $\lambda$ is the wave length, $R$ is the output impedance of each generator, $b$ is the distance between the monopoles, and $2 a$ is its diameter.

Accordingly the self $Z_{11}$ and the mutual $Z_{12}$ impedances of the radiators are:

$$
\begin{aligned}
Z_{11} & =Z_{22}=0.25\left(1 / Y_{1}+1 / Y_{2}\right) \\
& =Z_{m}\left(L, a_{e}\right)-j 0.25 W_{l} \cot k L+R, \\
Z_{12} & =0.25\left(1 / Y_{2}-1 / Y_{1}\right) \\
& =Z_{m}\left(L, a_{e}\right)+j 0.25 W_{l} \cot k L . .
\end{aligned}
$$

It is easy to show that in order to nullify the total field at the compensation point $\left(\rho_{0}+b, 0,0\right)$, i.e., $E_{z}(0)=E_{z 1}(0)+E_{z 2}(0)=0$, the ratio of the radiators' emf's must be equal to:

$$
\frac{e_{2}}{e_{1}}=-\frac{\left(Y_{2}+Y_{1}\right) F_{10}+\left(Y_{2}-Y_{1}\right) F_{20}}{\left(Y_{2}-Y_{1}\right) F_{10}+\left(Y_{1}+Y_{2}\right) F_{20}}
$$

here, $F_{m 0}$ is the value of function $F_{m}$ at the compensation point, where, $F_{m}$ is dependence of the field of the current excited with each generator from the distance to the observation point. Eq. (5) allows finding the voltage amplitude and phase at the input of the second radiator, if the amplitude and phase at the input of the first radiator are known: 


$$
J_{A 2} / J_{A 1}=-F_{10} / F_{20}
$$

i.e., the expression for required current ratio coincides with this expression in the absence of mutual coupling between the radiators. This fact occurs when the radiators are of the same length. For $L=\lambda / 4$, we obtain, in the first-order approximation:

$$
\frac{J_{A 2}}{J_{A 1}}=-\frac{R_{20}}{R_{10}} \exp \left[-j k\left(R_{10}-R_{20}\right)\right]
$$

where, $R_{20}=\sqrt{L^{2}+\rho_{0}^{2}}, R_{10}=\sqrt{L^{2}+\left(\rho_{0}+b\right)^{2}}$

As it follows from the Eq. (6), the dipole moments of linear radiators must be in inverse proportion to the distances from the radiators to the compensation point.

Fig. 3 shows two variants of the dark spot position relative to the head, where the spot boundaries are shown by a dotted line and the solid curve shows the head boundary. The feed points $A_{1}$ and $A_{2}$ of the main and the auxiliary radiators and the compensation point $\mathrm{C}$ in Fig. 3 lie on the structure axis, and hence the dark spot is symmetric relative to this axis. In Fig. $3 a$, the compensation point is placed outside the head, and in Fig. $3 b$ it is inside the head, close to its surface. In the latter case the whole dark spot lies inside the head, resulting in a significant reduction of losses inside the head.

The dark spot dimensions can be calculated using equations similar to Eq. (1) written for the radiator's field. The dark spot boundary is determined by radius $\rho_{n}$ depending on $b, z$ and $\varphi$, at which $n=\left|E_{z} / E_{z 10}\right|$ is equal to a given value $n_{0}$ (e.g., $n_{0}=0.1$ ), where $E_{z}$ is the total field and $E_{z 10}$ is the field of the main radiator. The intersection points $\rho_{1}$ and $\rho_{2}$ of curve $n$ with a given level $n_{0}$ determine the dark spot boundaries in the $\rho$ direction and the length $\rho_{1} \rho_{2}$ of the segment, along which $n$ is smaller than the required value $n_{0}$. The length $\Delta \rho=\rho_{2}-\rho_{1}$ of this segment, i.e., the dark spot length, is presented in Ref. [6] in the table and figures for different values of $b, \varphi$ and $n_{0}$, i.e., for different levels of field decrease at the dark spot boundary.

The calculations show that upon optimal design of the structure, in terms of the relative positions of the
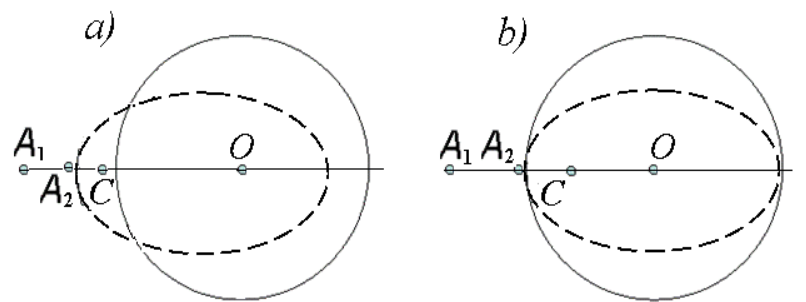

Fig. 3 The dark spot: compensation point is located outside (a) and inside (b) the head.

radiators and the compensation point, the volume of the dark spot increases, and the field inside this spot substantially decreases. One can choose the structure parameters so that the spot dimensions may coincide with the human head dimensions, resulting in a big reduction of losses power in the head, and in a high tolerance with respect to the user's head movements.

In Ref. [7], it is shown that using a second vertical auxiliary radiator placed along the symmetry axis of the structure, i.e., on the horizontal straight line passing through the head center $O$ and the compensation point, permits to increase the dark spot volume. Employing a few of auxiliary radiators allows a further increase of the dark spot volume. At the same time the irradiation reduction factor increases. This factor is defined as the ratio of the losses power in the absence and presence of the auxiliary radiator within the limits of the dark spot.

It is assumed, for the sake of simplicity, that the electric field strength increases linearly from the compensation point $t=0$ to the dark spot boundary $t=$ $t_{0}$ such that $\left|E_{z} / E_{z 10}\right|=n s$, where $s=t / t_{0}, n=\operatorname{const}(s)$, and hence the losses power exhibits a quadratic growth with the distance. The total losses power inside the spot is given by:

$$
P=P_{0} \int_{0}^{1}(n s)^{2} d s=P_{0} n^{2} / 3
$$

where, $P_{0}$ is the losses power due to the main radiator in the absence of the auxiliary radiators, within the limits of spot. For $n_{0}=0.2$ the losses power is diminished by a factor of $3 /(0.2)^{2}=75$ (or $18.8 \mathrm{~dB}$ ). For $n_{0}=0.1$ the reduction factor is $300(24.8 \mathrm{~dB})$, and for $n_{0}$ $=0.04$ the factor is $1,875(32.7 \mathrm{~dB})$. In practice, this factor is roughly half of the calculated value, since the field within the dark spot has a more complicated 
structure, i.e. the reduction factor is approximately equal to $1.5 / n_{0}^{2}$.

The pattern of the structure is considered in Ref. [8]. As said before, in order to preserve the circular form of the pattern, and in particular to avoid a deep gap along the structure symmetry axis, the auxiliary antenna field must be small compared to the main antenna field, i.e. this antenna must be placed at a minimal distance from the compensation point. If $J_{A 1}$ is the driving current of the main radiator, $E_{z 1}$ is its field, and $J_{A 1}=J_{A 2} D e^{-j \psi}$ is the driving current of the additional radiator, where $D$ is the ratio of the radiators' dipole moments, then the field of the additional radiator is $E_{2}=E_{1} D e^{j u}$, where, $u$ $=k l-\psi, l$ is the path-length of rays from the radiators to a point located at angle $\varphi$, and $\psi$ is the phase difference between the currents of the main and additional radiators.

Since the total field of both radiators is equal to $E=$ $E_{1}+E_{2}$, one can write for the pattern in the horizontal plane:

$$
|F|=|1+D \exp (j u)| / \sqrt{\left(1+D \cos u_{m}\right)^{2}+D^{2} \sin ^{2} u_{m}}(7)
$$

here, $u_{m}$ is the value of $u$ at the denominator maximum. Since, $D$ and $u_{m}$ are constant values, the denominator of this expression does not depend on $\varphi$, i.e., the ratio between the pattern maximum to the minimum is equal to:

$$
\frac{\left|F_{\max }\right|}{\left|F_{\min }\right|}=\frac{\left|1+D e^{j u}\right|_{\max }}{\left|1+D e^{j u}\right|_{\min }}=\frac{1 \pm D}{1 \mp D}
$$

where the upper sign corresponds to a positive $D$ and the bottom sign, to negative. It is easy to show that when the pattern maximum and minimum differ by 6 $\mathrm{dB}$ (deviation of $3 \mathrm{~dB}$ from the average level), we obtain $|D|=0.33$, and when the difference between the maximum and the minimum is $3 \mathrm{~dB}$, we get $|D|=$ 0.17 .

Taking in the first approximation that $R_{1}=\rho_{0}+b, R_{2}$ $=\rho_{0}$, we obtain $D=R_{2} / R_{1}=\rho_{0} /\left(\rho_{0}+b\right)$, which reduces to $\rho_{0}=b / \beta$, where $\beta=1 / D-1$. Consider the case when the main radiator is situated on the structure axis, at a distance of $3 \mathrm{~cm}$ from the head, i.e., $\rho_{0}+b=3$, and the compensation point is placed on the head surface. In this case, if $D=0.33$, then $\beta=2, \rho_{0}=b / 2=1, b=2$; for $D=0.17$ we have $\beta=4.88, \rho_{0}=0.5, b=2.5$. The results point out the necessity for a tradeoff between the irradiation reduction level and the radiation pattern of the two-antenna structure, especially in cases when the antenna platform is subject to spatial restrictions. However, the pattern changes are predictable and small, if the points of antennas displacement are selected correctly.

The simulation performed with the CST program show that the results of calculations, simulations and measurements are in good agreement, if the user's head is absent. The coincidence between the results in the presence of head is not too good (in accordance with the approximate estimation of permittivity), but a qualitative agreement does exist.

The obtained results show that under realistic conditions the compensation method allows reducing the irradiation power by a factor of 3-4 and more.

A method of user's irradiation reduction similar to the compensation method is proposed in [9], where the concrete ratio of two monopoles driving currents required for decreasing the SAR level at $2 \mathrm{GHz}$ is found by calculations using the Method of Moments. But in this paper an analysis of causes of an irradiation reduction and recommendations for the use of the observed effect are absent.

\section{Wideband Field Compensation}

Cellular communication is not the only area of application, where it is necessary to create a region of weak field near the transmitting antenna. The task is vital one, if, for example, a man uses or services a low-power radio station, which he carries or places nearby in a production area or in a vehicle, where it is necessary to protect from irradiation not only user but also other workers or passengers. Creating a weak field region in such cases is an efficient technique of 
protecting against irradiation.

In these cases, requirements to the produced weak field change in part. On the one hand, they often get complicated due to necessity of operation in a broad frequency range. On the other hand, the requirements become less stringent. For example, large dimensions of the transmitter allow increasing the room for mounting auxiliary radiators. Presence of a signal in the direction opposite to user's location sometimes is not required. The requirement for the horizontal pattern to be flat in the direction of user's location is somewhat lifted.

Operation in a broad frequency band gives rise to additional difficulties, since the fields of the main and the auxiliary radiators at the compensation point must have the same magnitude and opposite sign at each frequency. The phase shift can be easily implemented by using a transmitter output stage with balanced output. But, firstly, these two instances of the radiator type need to have identical characteristics at all frequencies of the operation range. Secondly, since the main and the auxiliary radiators are located at different distances from the compensation point, and signal velocities in the air and in the feed lines connecting the radiators to the transmitter are different, it is necessary to insert a small phase shifter in one channel. This phase shifter must provide the required delay time throughout the frequency range.

It is not easy to meet both these requirements. To provide the same signal amplitudes, it is necessary to secure identity of the main and the auxiliary radiators or to insert controlled attenuator in one channel. Wideband antennas often have a complicated structure, which uses different conducting and dielectric materials. That is why antenna characteristics are unstable, i.e., different antenna instances have different frequency responses, and so their fields are not equal. It is impossible to construct controllable attenuators in a wide frequency range.

Placing both radiators at equal distance from the compensation point yields better results.
A simple structure consisting of two radiators placed at the same distance from the compensation point is shown in Fig. 4. The radiators are two asymmetrical antenna instances of the same type, which have the circular pattern in the horizontal plane and similar amplitude-frequency responses. They are located at the same distance $b=a / \cos \beta$ from point $C$, where $a=A C$ is the distance from point $C$ to the middle line of the transmitter cover.

For example, the auxiliary radiator phase differ from the main radiator phase by $180^{\circ}$, and the amplitude of the vertically polarized signal $\vec{E}_{2}$ of the auxiliary radiator be $E_{2}=D E_{1}$, where $\vec{E}_{1}$ is the amplitude of the main radiator signal, and $D \leq 1$. If $D=1$, i.e., the amplitudes of the auxiliary signal and the main signal coincide, the sum of the signals in the plane of the structure symmetry is zero, since the signals in the plane are the same in amplitude and opposite in phase. If $D \neq 1$, the summing signal $E=E_{1}+E_{2}=(1-D) E_{1}$ is equal to a fixed small share of the main signal and weakly frequency dependent, since the distance from the radiators to the compensation point is the same, and the radiators themselves are similar. No phase shifter is needed in this case.

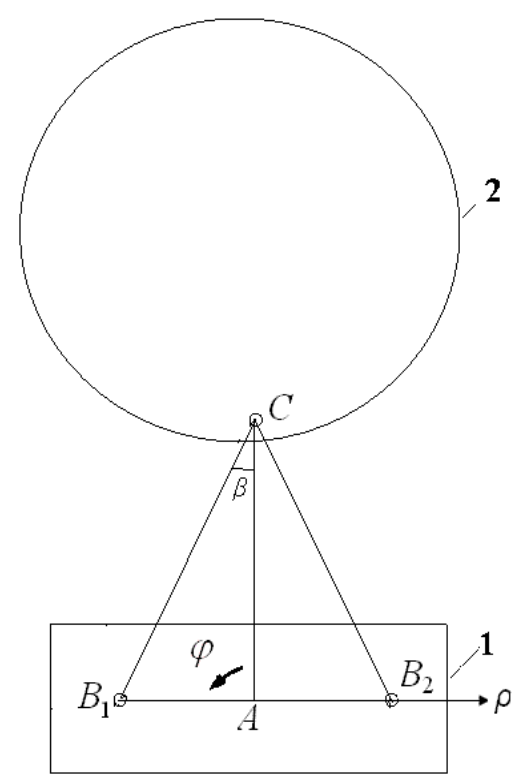

Fig. 4 The two-radiator structure. 1 is the transmitter top cover, 2 is the user's body, $B_{1}$ is the main radiator, $B_{2}$ is the auxiliary one, and $C$ is the compensation point. 
A disadvantage of the proposed circuit is the absence of the far field signal in the structure symmetry plane (in both directions), when $E_{2}=E_{1}$. But, first, this is not critical in many applications. Second, the angle (gap width), in which radiation signal is nearly zero, is very small. And third, the influence of the ground and neighboring bodies results in a smoothing of the pattern, i.e., both the depth and the width of the gap in the horizontal pattern will be reduced in real conditions.

Estimate the gap width by calculating angle $\Delta \varphi$ between its boundaries, i.e., between the points corresponding to given small value $f_{1}$ of the pattern. If $a=25 \mathrm{~cm}$, and $\beta=30^{\circ}$, the signal at angle $\varphi$, which consists of two signals with equal amplitude $E_{1}$ and opposite phase created by radiators located at distance $d=2 a \tan \beta=28.9 \mathrm{~cm}$ from each other is equal to $E=$ $2 E_{1} \sin \left[(k d / 2) \sin \varphi\right.$ ], i.e., $E_{\max }=2 E_{1}$. Here, the cylindrical system of coordinates is used (Fig. 4). If $\varphi$ is small,

$$
f_{1}=\sin [(k d / 2) \sin \varphi] \approx k d \varphi / 2
$$

i.e., $\Delta \varphi=2 \varphi=4 f_{1} /(k d)$. In particular, if $f_{1}=0.1$, the wavelength $\lambda$ is $30 \mathrm{~cm}$, and $d=28.9 \mathrm{~cm}$, then $\Delta \varphi=$ $3.8^{\circ}$.

Another disadvantage is the fact that, when $D \neq 1$, the irradiation power reduction factor inside the dark spot decreases, as the total field, fails to vanish even at the compensation point. But the disadvantage is also inherent in the case of antenna placement at different distance from the compensation point, due to the limited dimensions of the transmitter top cover.

Experimental results for the signal magnitude at the compensation point are given in Fig. 5. Curve 1 corresponds to the signal of the main antenna placed at point $A$ (Fig. 4), and curve 2 corresponds to the signal of two antennas placed at points $B_{1}$ and $B_{2}$. As seen from the results, mounting both radiators at the same distance from the compensation point permits to ensure compensation in a wider frequency band. The auxiliary radiator decreases the signal magnitude at the compensation point by 10-15 $\mathrm{dB}$ (3-6 times) in the band from 1.7 to $2.7 \mathrm{GHz}$.

Using of two auxiliary radiators gives additional advantages. In this case, the amplitude of each auxiliary radiator signal may be smaller than that of the main radiator. One variant of this compensation structure is presented in Fig. 6 (top view) where the same notation is used.

If $E_{0}$ is the amplitude of the main radiator signal at the compensation point, the amplitude of each auxiliary radiator signal must be $E_{1}=E_{2}=E_{0} / 2$. In the cylindrical coordinate system, the origin of which coincides with point $A$, the ratio of the auxiliary signal to the main signal in direction $\varphi$ is:

$$
\begin{gathered}
E_{1}(\rho) / E_{0}(\rho)=0.5 \exp \left[j k\left(a_{1}-b_{1}\right)\right], \\
E_{2}(\rho) / E_{0}(\rho)=0.5 \exp \left[j k\left(a_{1}+b_{1}\right)\right]
\end{gathered}
$$

where, $E_{0}(\rho)$ is the main radiator field at distance $\rho$, $E_{\mathrm{i}}(\rho)$ is the field of the auxiliary radiator located at point $B_{\mathrm{i}}$, and $a_{1}+b_{1}$ are the path-length differences of the signals from the main and auxiliary radiators to the observation point. Here, $a_{1}=a(1-\cos \beta) \sin \varphi, b_{1}=$ $a \sin \beta \cos \varphi$ (these segments are marked in Fig. 6). The total signal is:

$$
E(\rho)=E_{0}(\rho)\left[1-\cos \left(k b_{1}\right) \exp \left(j k a_{1}\right)\right]
$$

The ratio of the total and the main signals is equal to

$$
\begin{aligned}
& |E(\rho)| /\left|E_{0}(\rho)\right|=\sqrt{\left[1-\cos \left(k b_{1}\right) \cos \left(k a_{1}\right)\right]^{2}+} \\
& +\cos ^{2}\left(k b_{1}\right) \sin ^{2}\left(k a_{1}\right)
\end{aligned}
$$

In the structure symmetry plane, the total signal in the far region is:

$$
\begin{aligned}
& E(\rho) / E_{0}(\rho)=1-\exp [j k a(1-\cos \beta)]= \\
& =-2 j \exp [j k a(1-\cos \beta) / 2] \sin [k a(1-\cos \beta) / 2] \\
& \text { i.e., } \\
& \qquad\left|E(\rho) / E_{0}(\rho)\right|=2 \sin [k a(1-\cos \beta) / 2]
\end{aligned}
$$

If, for example, $a=25 \mathrm{~cm}$ and $\beta=30^{\circ}$ or $60^{\circ}$, we obtain at $1 \mathrm{GHz} \mid E(\rho) / E_{0}(\rho \mid=0.69$ and 1.22, respectively. The total far field in the symmetry plane in both directions is other than zero. It follows from 


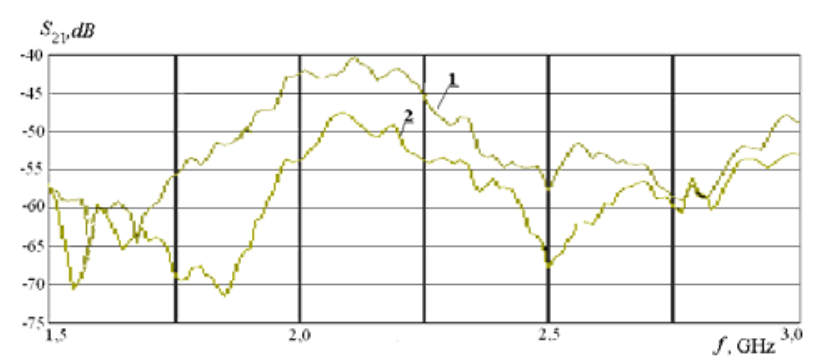

Fig. 5 The frequency dependence of the signal at the compensation point, when the main radiator (1) and two radiators (2) are placed at the same distances from the point.

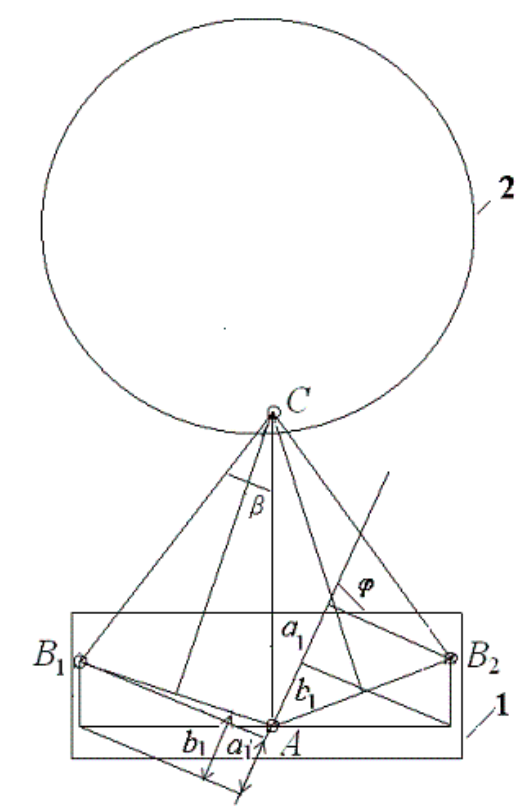

Fig. 6 The three-radiator structure.

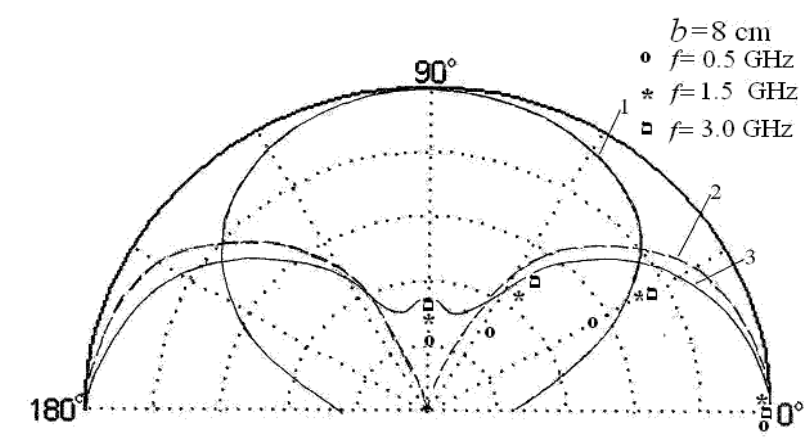

Fig. 7 The horizontal patterns of different structures at 1 GHz.

Eq. (6) that at any $\varphi$ the total signal in the far region is other than zero, too. This is an inherent advantage of such structure.

The calculated horizontal patterns at frequency $1 \mathrm{GHz}$ of one radiator placed at point $A$, of two radiators (Fig. 4) and of three radiators (Fig. 6) located on the cover of infinite dimensions are given in Fig. 7 and marked with symbols 1,2 and 3, respectively.

The considered compensation structures comprise radiators placed at the same distances from the compensation point. The structures require no use of any phase shifter. The compensation structures, in which flat metal reflectors (mirrors) are used instead of auxiliary radiators, permit to create a weak field in the subspace on the side of the reflectors, where the antenna is located, which is similar to the one generated by three radiators.

An example of such structure is presented in Fig. 8 (top view). As seen from the figure, the flat metal plate, in which main radiator $A$ is reflected, is mounted on the transmitter top cover along the structure axis of symmetry (in the vertical plane passing through compensation point $C$ ). The reflection (or imaging) of the main radiator signal by the metal plate into the space in front of the reflector is equivalent to the presence of second radiator $B$ located at the same distance behind the plate.

The reflected signal phase differs from the main radiator phase by $180^{\circ}$, since vector $\vec{E}_{1}$ of the incident signal is located along the tangent to the reflector surface.

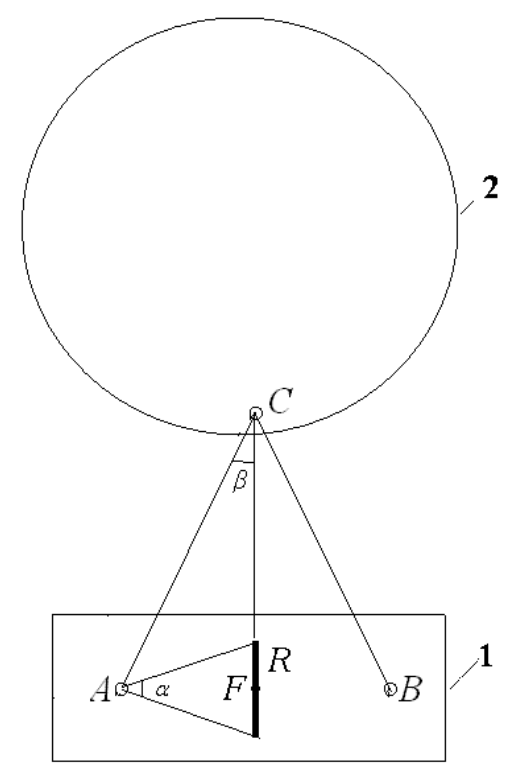

Fig. 8 The structure with one reflector. $R$ is the metal reflector, and $B$ is the location of the equivalent radiator. 
The amplitude of the reflected signal $\vec{E}_{2}$ is $E_{2}=D E_{1}$, where $D$ depends on magnitude $\alpha$ of the angle, subtended at point $A(D \leq 1)$ by the reflector.

If the plate dimensions are infinite (or if they are much larger in all directions than the main radiator dimensions), then $D=1$, i.e., the amplitudes of the reflected and the main signal coincide, and the total signal in the reflector plane is zero. If $D \neq 1$, total signal $E=E_{1}+E_{2}=(1-D) E_{1}$ is a fixed share of the main signal, and the share is independent of frequency, since the distances from both radiators to the compensation point are the same, and the radiators themselves are identical.

On the whole, the performance of the structure is similar to that of the two-radiator structure presented in Fig. 4. No phase shifter is needed in this structure. An additional and very important advantage of the described structure is that the external actions, e.g., an approach of metal bodies, exert practically no effect on the structure operation, as the main radiator and the reflected signals undergo the same changes upon any external actions. Only the appearance of a metal body between a signal source and the compensation point is an exception and a very rare one at that.

The influence of a mirror (a metal plate) on the antenna input impedance decreases the matching between the antenna and the transmitter. To offset the decreased matching, it is necessary to increase the distance between the antenna and the mirror as well as the mirror width so that the second signal would not decrease. The reflector width has only a weak effect on the antenna input impedance, but essentially increases signal $\vec{E}_{2}$. Moreover, the mirror influence on the antenna input impedance is constant and can be allowed for a priori. The mirror height must exceed that of the antenna.

Flat reflectors can be manufactured in the form of a light-weight, strong and collapsible construction (if necessary). In such a case, each reflector is implemented as a set of vertical parallel wires, located at a distance of $0.06 \lambda_{\min }$, where, $\lambda_{\min }$ is the minimum wavelength in the frequency range.

Using two reflectors, i.e., creating two equivalent radiators, gives additional advantages. In this case, the amplitude of each reflected signal may be smaller than that of the main radiator signal, and so the requirements to the reflector loosen. A variant of such compensation structure is presented in Fig. 9 (top view), where, as before, $B_{1}$ and $B_{2}$ denote the equivalent radiators locations. The performance of the structure of Fig. 9 is similar to that of the three-radiator structure presented in Fig. 6: the distance from all radiators to the compensation point is the same, i.e., field variations due to frequency dependence have no effect on the compensation quality.

As stated above, the amplitude of the reflected signal (vertically polarized) is $E_{2}=D E_{1}$. Here, $D$ depends on the mirror dimensions.

An essential drawback of the structure with two reflectors is that the reflectors creating the dark spot in the near region block at the same time prevent the electromagnetic waves in the angular sector, shadowed by them. Here, the signal in the open sector increases

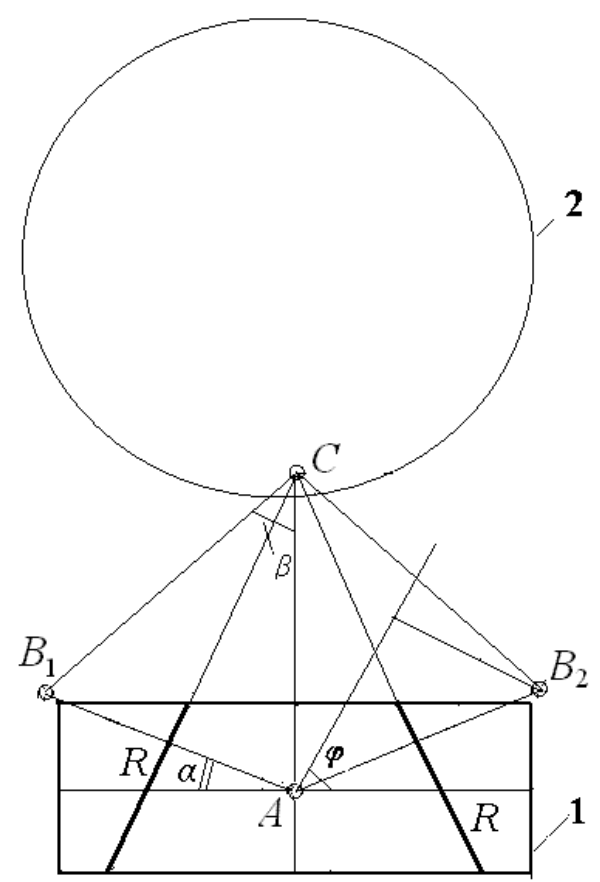

Fig. 9 Compensation structure with two reflectors. 
approximately twice. This drawback limits the application of such structures.

The structure with two reflectors was tested experimentally. A general view of the experimental setup is presented in Fig. 10. The following notation is used in the figure: 1 is the radiator, 2 is the receiving antenna, placed at the compensation point, and 3 and 4 are flat reflectors, located symmetrically on either side of the straight line passing through the compensation point and the radiator. The distance between the radiator and the receiving antenna is $25 \mathrm{~cm}$. The reflector is manufactured in the shape of a vertical rectangular plate. Contact with the ground is maintained along the whole width of the reflector, using a horizontal element. In the course of the measurements, each reflector was separately moved along a straight line to the point where the received signal at $2 \mathrm{GHz}$ (close to the band center) decreased by $6 \mathrm{~dB}$. As a result the antenna field at the compensation point in the presence of both reflectors is substantially smaller than the field of the antenna without reflectors. The measurements are performed at two different values of reflectors width $b$, i.e., 8 and $15 \mathrm{~cm}$, and at different angles $\left(10^{\circ}, 15^{\circ}\right.$ and $\left.20^{\circ}\right)$ between the axis of the setup symmetry and the lines, along which the reflectors were moved to attain the zero field at the

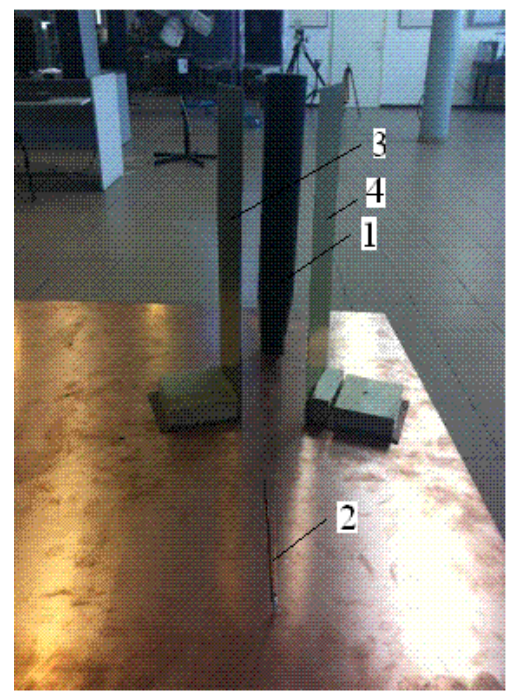

Fig. 10 General experimental setup.

Table 1 The measurement results for the signal magnitudes at the compensation point, in dB.

\begin{tabular}{|c|c|c|c|c|c|c|c|}
\hline \multirow{3}{*}{$f(\mathrm{GHz})$} & \multirow{3}{*}{ Without reflector } & \multicolumn{3}{|c|}{ With reflectors of width $(\mathrm{cm})$} & \multicolumn{3}{|c|}{ Difference } \\
\hline & & \multicolumn{2}{|c|}{8} & \multirow{2}{*}{$\frac{15}{15}$} & \multicolumn{2}{|r|}{8} & \multirow{2}{*}{$\begin{array}{l}15 \\
15\end{array}$} \\
\hline & & $\beta / 2=10^{\circ}$ & 15 & & 10 & 15 & \\
\hline 0.5 & -17 & -32.1 & -33.3 & -35.8 & 15.1 & 16.3 & 18.8 \\
\hline 1.0 & -19.5 & -33.5 & -35.8 & -34.3 & 14 & 16.3 & 14.8 \\
\hline 1.5 & -39 & -46.4 & -43.4 & -54.9 & 7.4 & 4.4 & 15.9 \\
\hline 2.0 & -28.8 & -37.3 & -40.4 & -62.5 & 8.5 & 11.6 & 33.7 \\
\hline 2.5 & -27.7 & -47.1 & -42.6 & -41.3 & 19.4 & 14.9 & 13.6 \\
\hline 3.0 & -36.3 & -43.9 & -47 & -42.6 & 7.6 & 10.7 & 6.3 \\
\hline
\end{tabular}
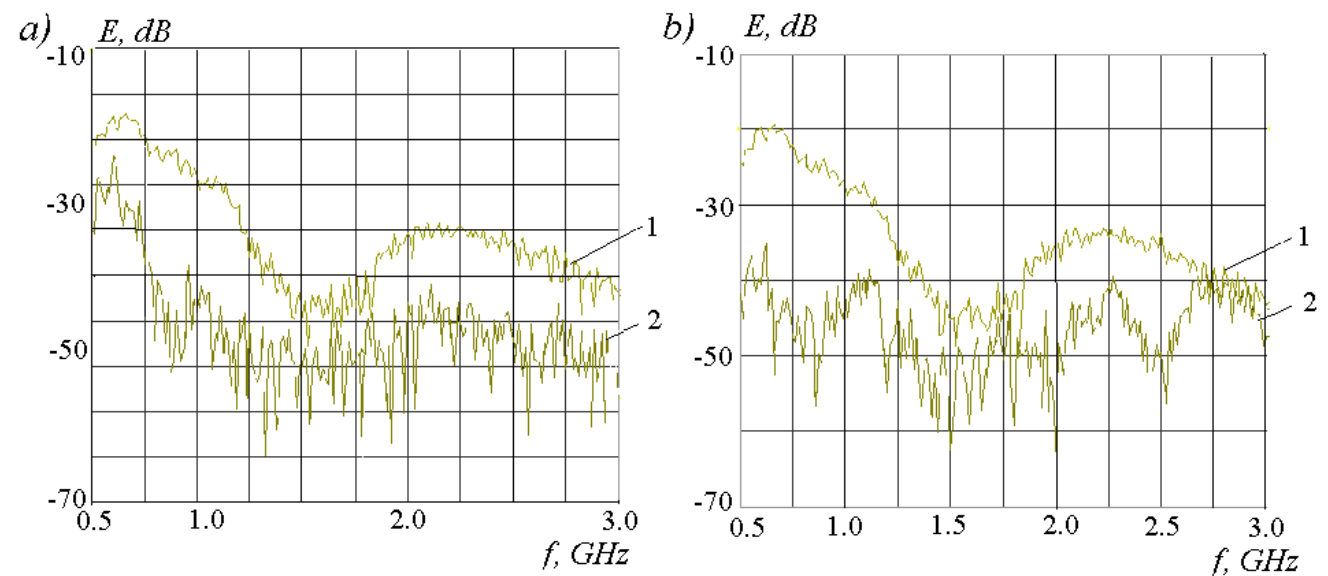

Fig. 11 Frequency dependence of signal at the compensation point without reflectors (1) and with reflectors (2) for reflector width (a) $8 \mathrm{~cm}$ and (b) $15 \mathrm{~cm}$. 
compensation point. In all variants, when the distance between the compensation point and the near edge of the reflector was $9 \mathrm{~cm}$, the signal was half as strong as its value without the reflector.

The measurement results are given in Table 1. The frequency dependence of the signal at the compensation point without reflectors (1) and with reflectors (2) is presented in Fig. 11, for reflector widths of 8 (a) and $15 \mathrm{~cm}$ (b). As it is clear from the table and figures, the reflectors with width $8 \mathrm{~cm}$ permit one to decrease the signal magnitude without tuning by $10-15 \mathrm{~dB}$ for the whole frequency range, and the reflectors with width $15 \mathrm{~cm}$ perform even better.

The dark spot boundaries of the three radiator structure (Fig. 6) in the horizontal plane $(z=0)$, which are determined by the procedure described above, show that these spots have sufficiently large dimensions.

\section{Conclusions}

We have analyzed a compensation method for wideband operation. Different structures for wideband compensation are introduced and investigated, for example, replacing the phase shifter by a delay line. The calculation and experimental results show that using several radiators located at equal distances from the compensation point or using flat reflectors, one can decrease the irradiation power by a factor of 5 or more in a large bandwidth. Two auxiliary radiators or the flat reflectors permit to achieve these results without introducing deep and wide gaps in the horizontal pattern.

\section{References}

[1] M. Bank, B. Levin, The development of the cellular phone antenna with a small radiation of human organism tissues, IEEE Antennas Propagat Magazine 49 (4) (2007) 65-73.

[2] B.Z. Katzenelenbaum, B.M. Levin, Synthesis of antennas, in which near region there is the weak field area, Antennas 97 (6) (2005) 38-46. (in Russian)

[3] B.M. Levin, Formation of a weak-field zone near a wire antenna, Journal of Communications Technology and Electronics 51 (6) (2006) 660-662.

[4] B.M. Levin, Calculation of electrostatic field in heterogeneous media, Journal of Communications Technology and Electronics 42 (8) (1997) 852-857.

[5] B.M. Levin, A long line and a nonsymmetric oscillator with wires of different length, Journal of Communications Technology and Electronics 57 (11) (2012) 48-55.

[6] B. Levin, M. Haridim, M. Bank, Cellular phone antenna with a small radiation of human head, IEEE Antennas Propagat. Magazine 55 (4) (2013) 113-127.

[7] M. Haridim, B. Levin, M. Bank, Fields' compensation by two linear arrays, in: 15th Intern. Seminar/Workshop DIPED-2010, Tbilisi, 2010, pp. 156-159.

[8] B. Levin, M. Bank, M. Haridim, The compensation method and the antenna pattern, in: 15th Intern. Seminar/Workshop DIPED-2010, Tbilisi, 2010, pp. 160-163.

[9] M.A. Mangoud, R.A. Abd-Alhameed, N.J. McEwan, P.S. Excell, E.A. Abdulmula, SAR reduction for handset with two-element phased array antenna computed using hybrid MoM/ FDTD technique, Electronic Letters 35 (20) (1999) 1693-1694. 


\title{
$H^{ \pm}$Decays into $W^{ \pm}$and $a_{1}$ in the Next Minimal
} Supersymmetric Standard Model

\author{
Nguyen Chinh Cuong \\ Faculty of Physics, Hanoi National University of Education, Hanoi 84-4, Vietnam
}

Received: August 21, 2013 / Accepted: September 26, 3013 / Published: February 15, 2014.

\begin{abstract}
To solve the $\mu$ problem of the Minimal Supersymmetric Standard Model (MSSM), a single field S is added to build the Next Minimal Supersymmetric Standard Model (NMSSM). Vacuum enlarged with non-zero vevs of the neutral-even CP is the combination of $\mathrm{H}_{\mathrm{u}}, \mathrm{H}_{\mathrm{d}}$ and S. In the NMSSM, the Higgs sector is increased to 7 (compared with 5 hogs in the MSSM), including three Higgs-which are the even-CP $\mathrm{h}_{1,2,3}\left(\mathrm{~m}_{\mathrm{h} 1}<\mathrm{m}_{\mathrm{h} 2}<\mathrm{m}_{\mathrm{h} 3}\right)$, two Higgs-which are odd-CP $\mathrm{a}_{1,2}\left(\mathrm{~m}_{\mathrm{a} 1}<\mathrm{m}_{\mathrm{a} 2}\right)$ and a couple of charged Higgs $\mathrm{H}^{ \pm}$. The decays Higgs into Higgs is one of the remarkable new points of the NMSSM. In this paper, we study the decays $\mathrm{H}^{ \pm}$into $\mathrm{W}^{ \pm}$and $\mathrm{a}_{1}$. The decay width is calculated to one loop vertex correction. The numerical results are also described together with evaluations.
\end{abstract}

Key words: Higgs, decays, NMSSM.

\section{Introduction}

The simplest version of supersymmetry is the Minimal Supersymmetric Standard Model (MSSM). This version is limited by two problems: the $\mu$ and the hierarchy [1-4]. The simple supersymmetry, which is beyond the MSSM, is the Next Minimal Supersymmetric Standard Model (NMSSM). The special characteristic of Higgs boson in the NMSSM is the decay of Higgs into Higgs. It is remarkable that the lightest state a1 of the odd-CP Higgs (CP is Charged and Parity transition) can play a role of a pseudo, goldstone, which has small mass and can lead to the predominated decay of the even-CP $h \rightarrow a_{1} a_{1}$ [5]. The even-CP Higgs and the heavy odd-CP Higgs can be generated at Large Electron-Positron Collider (LEP) in $\mathrm{e}^{+} \mathrm{e}^{-} \rightarrow$ ha , but they may not be discovered because the dominant h decay was not searched for. There are different ways to make the mass of Higgs boson

Corresponding author: Nguyen Chinh Cuong, research fields: CP violation, super symmetry standard models, simulation. E-mail: cuongnc@hnue.edu.vn. increased in the MSSM and in the beyond MSSM. One simple way is to study the beyond singlet of the MSSM which contains one term $\lambda \hat{\mathrm{S}} \hat{\mathrm{H}}_{\mathrm{u}} \hat{\mathrm{H}}_{\mathrm{d}}$ in the super-potential, this is the term that contributes $\lambda^{2} \mathrm{v}^{2} \sin ^{2} 2 \beta$ at $\mathrm{v}=174 \mathrm{GeV}$ to the squared mass of even-CP Higgs [6] and therefore, it can make the mass of Higgs boson increased over the limit of independent decay state. It should be noted that this contribution is maximum with $\tan \beta \sim 1$. Thus, a condition in which the lightest odd-CP Higgs with its mass is under $2 \mathrm{~m}_{\mathrm{b}}$, the two lightest even-CP Higgs boson and the charged Higgs boson can be found in the MSSM. All of them can be generated at LEP and they are now being searched for.

The charged Higgs makes up more than $40 \%$ in the top-quark decay at Tevatron; the products of this decay are charged Higgs and bottom-quark $\left(\mathrm{t} \rightarrow \mathrm{H}^{+} \mathrm{b}\right)$. The decay method of charged Higgs is $\mathrm{H}^{ \pm} \rightarrow \mathrm{W}^{ \pm} \mathrm{a}_{1}$, with $\mathrm{a}_{1} \rightarrow \mathrm{c} \overline{\mathrm{c}}$, gg .

The neutral Higgs sector in the NMSSM includes the following states: three even-CP and two odd-CP. Many 
analysis on Higgs sector in the NMSSM [7] have shown that, in the specific physical state of the even-CP Higgs, there is a strong mix between the doublet state and the singlet $\mathrm{SU}(2)$ with the reduction in the interaction of gauge boson. The study on light Higgs contributes to the discovery of one or more Higgs states at LEP, at Large Hadron Collider (LHC) [7] and at large energy accelerators.

The NMSSM is established from the MSSM when the MSSM is added with a gauge chiral single superfield $\hat{S}$, which includes the renormalized superfield interaction and the soft supersymmetry breaking term $L_{S o f t}$. In the NMSSM, the terms of the super-potential $\mathrm{W}_{\text {Higgs }}$ are dependent on superfield Higgs $\hat{H}_{d}, \hat{H}_{u}$ and $\hat{S}$ (here, we follow the SLHA2 regulations, however $\hat{H}_{u}$ is also written as $\hat{H}_{2}$ and $\hat{H}_{d}$ is also written as $\hat{H}_{1}$ ):

$$
W_{\text {Higgs }}=(\mu+\lambda \hat{S}) \hat{H}_{u} \cdot \hat{H}_{d}+\xi_{F} \hat{S}+\mu^{\prime} \hat{S}^{2}+\frac{\kappa}{3} \hat{S}^{3}(1)
$$

with: $\lambda, \kappa$ is the non-dimension coupling Yukawa; $\mu, \mu^{\prime}$ is the supersymmetry mass; $\xi_{\mathrm{F}}$ is the square supersymmetry mass parameter.

From Eq. (1), Yuakawa interaction of quark and lepton superfield are added to:

$$
W_{\text {Yukawa }}=h_{u} \hat{H}_{u} \cdot \hat{Q} \hat{U}_{R}^{c}+h_{d} \hat{H}_{d} \cdot \hat{Q} \hat{D}_{R}^{c}+h_{e} \hat{H}_{d} \cdot \hat{L} \hat{E}_{R}^{c}(2)
$$
here, the Yukawa interaction $h_{u}, h_{d}, h_{e}$ and the superfields $\hat{Q}, \hat{U}_{R}^{c}, \hat{D}_{R}^{c}, \hat{L}, \hat{E}_{R}^{c}$ are the matrices and vectors in the corresponding spaces.

The soft breaking supersymmetry sector is regulated in SLHA2:

$$
\begin{aligned}
& -L_{s o f t}=m_{H u}^{2}\left|H_{u}\right|^{2}+m_{H d}^{2}\left|H_{d}\right|^{2}+m_{s}^{2}|S|^{2}+m_{Q}^{2}\left|Q^{2}\right| \\
& +m_{U}^{2}\left|U_{R}^{2}\right|+m_{D}^{2}\left|D_{R}^{2}\right|+m_{L}^{2}\left|L^{2}\right|+m_{E}^{2}\left|E_{R}^{2}\right| \\
& +\left(h_{u} A_{u} Q \cdot H_{u} U_{R}^{c}-h_{d} A_{d} Q \cdot H_{d} D_{R}^{c}-h_{e} A_{e} L \cdot H_{d} E_{R}^{c}\right. \\
& \left.+\lambda A_{\lambda} H_{u} \cdot H_{d} S+\frac{1}{3} \kappa A_{\kappa} S^{3}+m_{3}^{2} H_{u} \cdot H_{d}+m_{s}^{2 \prime} S^{2}+\xi_{s} S+h c\right)
\end{aligned}
$$

(definition $\mathrm{m}_{3}^{2}=\mathrm{B} \mu, \mathrm{m}_{\mathrm{s}}^{\prime 2}=\mathrm{B}^{\prime} \mu^{\prime}$ ).

In the super-potential Eq. (1), we have supersymmetry parameters $\mu, \mu$ ' and $\xi_{F}$ (the soft supersymmetry interactions break the parameters $m_{3}^{2}, m_{\mathrm{S}}^{\prime 2}$ and parameter $\xi_{\mathrm{S}}$ in Eq. (3)), however, some terms are not eliminated in some different solutions for simple NMSSM with a part of invariant super-potential when $\mu=\mu^{\prime}=\xi_{\mathrm{F}}=0$

$$
W_{N M S S M}=\lambda \hat{S} H_{u} \cdot H_{d}+\frac{\kappa}{3} \hat{S}^{3}
$$

Then, eliminating the parameters $m_{3}^{2}, m_{\mathrm{S}}^{\prime 2}$ and $\xi_{S}$ in Eq. (3), combining the vevs of $\hat{S}$ in the weak sector or in the breaking supersymmetry to define $\mu$ :

$$
\mu_{\text {eff }}=\lambda \mathrm{s}
$$

The matter of $\mu$ in MSSM has been solved then.

As any supersymmetry theory with invariant super-potential sector (ternary), the Lagrangians, which contain the soft supersymmetry violation conditions specified by Eq. (3), have one $Z_{3}$ symmetry randomly, which is corresponding to the multiplication of all chiral superfields with $\mathrm{e}^{2 \pi \mathrm{i} / 3}$. The invariant super-potential Eq. (4) is presented like invariant $Z_{3}$. The non-dimension terms in the super-potential Eq. (1) will break the symmetry $Z_{3}$. The model with super-potential Eq. (1) is the NMSSM. The invariant $\mathrm{Z}_{3}$ Higgs sector is defined by the seven parameters $\lambda, \kappa, m_{H_{d}}^{2}, m_{H_{u}}^{2}, m_{S}^{2}, A_{\lambda}, A_{\kappa}$. The expressions of Higgs mass matrix in the invariant $Z_{3}$ of the NMSSM show that invariant $Z_{3}$ is obtained when:

$$
m_{3}^{2}=m_{S}^{2 \prime}=\xi_{S}=\mu=\mu^{\prime}=\xi_{F}=0
$$

From the supersymmetry gauge interaction and soft supersymmetry breaking conditions, we obtain Higgs potential:

$$
\begin{aligned}
V_{\text {Higgs }}= & \left|\lambda\left(H_{u}^{+} H_{d}^{-}-H_{u}^{0} H_{d}^{0}\right)+\kappa S^{2}+2 \mu^{\prime} S+\xi_{F}\right|^{2} \\
& +\left(m_{H_{u}}^{2}+|\mu+\lambda S|^{2}\left(\left|H_{u}^{0}\right|^{2}+\left|H_{u}^{+}\right|^{2}\right)\right. \\
& +\left(m_{H_{d}}^{2}+|\mu+\lambda S|^{2}\left(\left|H_{d}^{0}\right|^{2}+\left|H_{d}^{-}\right|^{2}\right)\right. \\
& +\frac{g_{1}^{2}+g_{2}^{2}}{8}\left(\left|H_{u}^{0}\right|^{2}+\left|H_{u}^{+}\right|^{2}-\left|H_{d}^{0}\right|^{2}-\left|H_{d}^{-}\right|^{2}\right)^{2} \\
& +\frac{g_{2}^{2}}{2}\left|H_{u}^{+} H_{d}^{0^{*}}+H_{u}^{0} H_{d}^{0^{*}}\right|^{2}+m_{S}^{2}|S|^{2} \\
& +\left(\lambda A_{\lambda}\left(H_{u}^{+} H_{d}^{-}-H_{u}^{0} H_{d}^{0}\right) S+\frac{1}{3} \kappa A_{\kappa} S^{3}\right. \\
& +m_{3}^{2}\left(H_{u}^{+} H_{d}^{-}-H_{u}^{0} H_{d}^{0}\right)+m_{S}^{\prime 2} S^{2}+\xi_{S} S+h . c
\end{aligned}
$$


where $g_{1}$ and $g_{2}$ present gauge interaction $U(1)$ and $S U(2)$.

The even-CP Higgs sector has three mixed states, which are the real parts of $H_{u}, H_{d}$ and $S$. The largest mass state $h$, which is nearly the same with SM, interacts with electroweak Gauge boson, this state has squared mass $\mathrm{M}_{\mathrm{h}}^{2}$ given by [7, 8]:

$$
M_{h}^{2}=M_{z}^{2} \cos ^{2} 2 \beta+\lambda^{2} v^{2} \sin ^{2} 2 \beta+\text { rad.corrs }+\Delta_{\operatorname{mix}}(8)
$$

The diagonal elements of matrix in the singlet sector are given by (with the assumption that $\mathrm{s}>\mathrm{v}_{\mathrm{u}}, \mathrm{v}_{\mathrm{d}}$ )

$$
M_{s s}^{2}=k s\left(A_{k}+4 k s\right)
$$

The term $\Delta_{\text {mix }}$ in Eq. (8) is derived from the mixture of doublet and singlet

$$
\Delta_{m i x}=\frac{4 \lambda^{2} s^{2} v^{2}(\lambda-k \sin 2 \beta)^{2}}{\bar{M}_{h}^{2}-M_{S S}^{2}}
$$

where, $\bar{M}_{h}^{2}$ is given by $\mathrm{M}_{\mathrm{h}}^{2}$ and has no mixed term.

And the mass of charged Higgs boson is defined by

$$
M_{ \pm}^{2}=M_{P, A A}^{2}+v^{2}\left(\frac{g_{2}^{2}}{2}-\lambda^{2}\right)
$$

The process of the decay of Higgs into Higgs also contains other interests; that is the decay of one charged Higgs $\mathrm{H}^{ \pm}$into $\mathrm{W}^{ \pm}+\mathrm{a}_{1}$ (or $\mathrm{W}^{ \pm}+\mathrm{h}$ ) with $\mathrm{a}_{1} \rightarrow$ gg, $\overline{\mathrm{cc},} \tau^{+} \tau^{-}$, this is the channel that was missed in the process of searching for the decays $\tau \rightarrow \mathrm{H}^{+} \mathrm{b}$ at Tevatron. In the MSSM, the mass of charged Higgs is related to the mass of the particle $\mathrm{W}$ and odd-CP Higgs.

$$
m_{H^{ \pm}}^{2}=m_{W^{ \pm}}^{2}+m_{A}^{2}+(\text { SUSY loop })
$$

Supersymmetry (SUSY) does not contribute much in this relationship, since $\mathrm{m}_{\mathrm{A}}$ is minor, we can see that $\mathrm{m}_{\mathrm{H}^{ \pm}} \approx \mathrm{m}_{\mathrm{w}^{ \pm}}$. The dominant decay process of charged Higgs is $\mathrm{H}^{+} \rightarrow \mathrm{W}^{+} \mathrm{a}_{1}$, which is nearly $100 \%$, except when the mass of charged Higgs almost reaches to the mass of Boson $\mathrm{W}^{+}$. In that case, the decay process $\mathrm{H}^{+} \rightarrow \mathrm{W}^{+} \mathrm{a}_{1}$ is still dominant, $\mathrm{B}\left(\mathrm{H}^{+} \rightarrow \mathrm{W}^{+} \mathrm{a}_{1}\right) \geq 70 \%$

The searching for charged Higgs which decays into
$\mathrm{W}^{ \pm} \mathrm{a}_{1}$ is relatively easy at LHC, where quark top produces charged Higgs ( $\left.\tau \rightarrow \mathrm{H}^{+} \mathrm{b}\right)$. Studying the decay of new particles in the Model will bring us the hope of finding out these particles as well as verifying the correctness of that Model [9]. In this paper, we study the decay $\mathrm{H}^{ \pm} \rightarrow \mathrm{W}^{ \pm} \mathrm{a}_{1}$ and calculate the decay width to one loop vertex correction. The numerical results are also shown in graph to evaluate the influence of $\mathrm{m}_{\mathrm{a}_{1}}$ to the decay width and to the lifetime of $\mathrm{H}^{ \pm}$.

\section{The Decay $\mathrm{H}^{ \pm} \rightarrow \mathrm{W}^{ \pm} \mathrm{a}_{1}$}

The amplitude which is calculated to tree level has the following form:

$$
M_{0}=\varepsilon_{\mu}(\vec{k}, \lambda) \cdot \frac{g}{2}\left(\sin \beta \cdot U_{11}^{P}+\cos \beta \cdot U_{12}^{P}\right)\left(q+q_{2}\right)^{\mu} .
$$

The decay width which is calculated to tree level is:

$$
\Gamma_{0}\left(H^{ \pm} \rightarrow W^{ \pm} a_{1}\right)=\frac{\left|M_{0}\right|^{2} \lambda}{16 m_{H}^{3} \pi}
$$

with

$$
\begin{aligned}
&\left|M_{f i}\right|^{2}=- \frac{g^{2}}{4}\left(\sin \beta \cdot U_{11}^{P}+\cos \beta \cdot U_{12}^{P}\right)^{2}\left(2 m_{H}^{2}+2 m_{a_{1}}^{2}-m_{W}^{2}\right) \\
&+\frac{g^{2}}{4}\left(\sin \beta \cdot U_{11}^{P}+\cos \beta \cdot U_{12}^{P}\right)^{2} \frac{\left(m_{H}^{2}-m_{a_{1}}^{2}\right)^{2}}{m_{W}^{2}}, \\
& \text { and } \lambda=\sqrt{m_{H}^{4}+m_{W}^{4}+m_{a_{1}}^{4}-2 m_{H}^{2} m_{W}^{2}-2 m_{W}^{2} m_{a_{1}}^{2}-2 m_{H}^{2} m_{a_{1}}^{2}}
\end{aligned}
$$

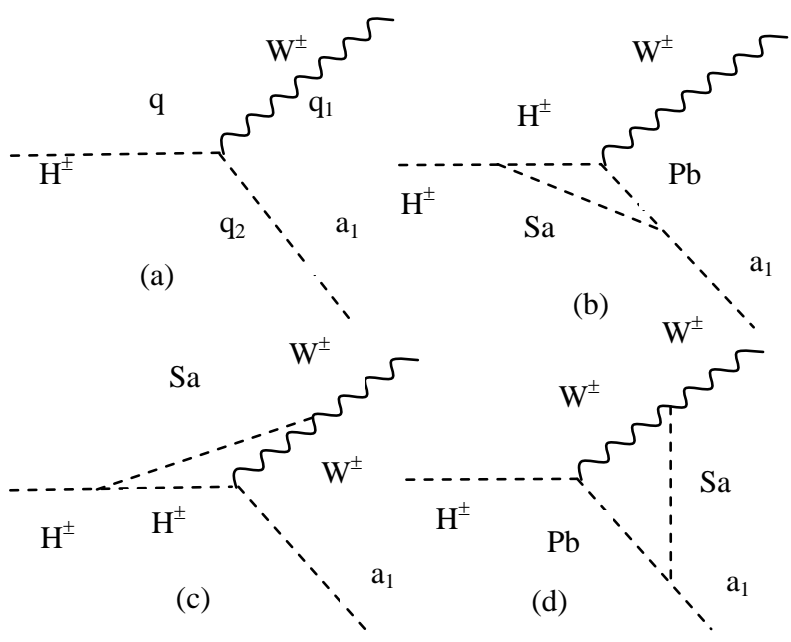

Fig. 1 Feyman diagram for correction SUSY-QCD in decay $\mathrm{H}^{ \pm} \rightarrow \mathrm{W}^{ \pm} \mathrm{a}_{1}$. (a) Tree level; (b), (c) and (d) one loop vertex correction. 
The decay width which is calculated to one loop vertex correction has the following result:

$$
\Gamma\left(H^{ \pm} \rightarrow W^{ \pm} a_{1}\right)=\frac{\left|M_{f i}\right|^{2} \lambda}{16 m_{H}^{3} \pi}
$$

with $\left|M_{f i}\right|^{2}=M_{0}^{+} \cdot M_{0}+2 \operatorname{Re}\left(M_{0}^{+} \cdot \delta M_{1}+M_{0}^{+} \cdot \delta M_{2}+M_{0}^{+} \cdot \delta M_{3}\right)$ $M_{0}^{+} \cdot \delta M_{1}=\frac{A \cdot B}{(2 \pi)^{4}} \frac{g^{2} \pi^{2}}{4}\left(\sin \beta \cdot U_{11}^{P}+\cos \beta \cdot U_{12}^{P}\right)^{+}\left(\sin \beta \cdot U_{b 1}^{P}+\cos \cdot U_{b:}^{F}\right.$

$\left\{\begin{array}{l}{\left[-\left(2 m_{H}^{2}+2 m_{a_{1}}^{2}-m_{W}^{2}\right)+\frac{1}{m_{W}^{2}}\left(m_{H}^{2}-m_{a_{1}}^{2}\right)^{2}\right] C_{0}(1)+} \\ +\left[-\left(m_{H}^{2}+3 m_{a_{1}}^{2}-m_{W}^{2}\right)+\frac{\left(m_{H}^{2}-m_{a_{1}}^{2}\right)\left(m_{H}^{2}-m_{a_{1}}^{2}-m_{W}^{2}\right)}{m_{W}^{2}}\right] C_{11}(1)\end{array}\right\}$

with $C_{0}(1)=C_{0}\left(m_{a_{1}}^{2}, m_{W}^{2}, m_{H}^{2}, m_{S a}^{2}, m_{P b}^{2}, m_{H}^{2}\right)$

and $C_{11}(1)=C_{11}\left(m_{a_{1}}^{2}, m_{W}^{2}, m_{H}^{2}, m_{S a}^{2}, m_{P b}^{2}, m_{H}^{2}\right)$ are the Pasarino-Vellman functions.

The vertex elements $A, B$, in which $S_{1} \equiv h_{1} ; S_{2} \equiv h_{2}$; $\mathrm{S}_{3} \equiv \mathrm{h}_{3}$ and $\mathrm{P}_{1} \equiv \mathrm{a}_{1} ; \mathrm{P}_{2} \equiv \mathrm{a}_{2}$,

$$
\begin{aligned}
& A=-i g m_{W}\left(U_{a 1}^{S} \cos \beta+U_{a 2}^{S} \sin \beta\right) \\
& -\frac{i g m_{W}}{2 \cos \theta_{W}}\left(U_{a 1}^{S} \sin \beta+U_{a 1}^{S} \cos \beta\right) \cdot \cos 2 \beta \\
& +i \frac{\lambda^{2}}{\sqrt{2}}\left(v_{1} U_{a 2}^{S}+v_{2} U_{a 1}^{S}\right) \sin 2 \beta \\
& -\frac{i}{\sqrt{2}} \lambda U_{a 3}^{S}\left[\left(2 k x+A_{\lambda}\right) \sin \beta+2 \lambda x\right] \text {, } \\
& B=-i \frac{g^{2}+g^{\prime 2}}{2 \sqrt{2}}\left(v_{1} U_{a 1}^{S} U_{11}^{P} U_{b 1}^{P}+v_{2} U_{a 2}^{S} U_{12}^{P} U_{b 2}^{P}\right) \\
& +\left(i \frac{g^{2}+g^{\prime 2}}{2 \sqrt{2}}-\sqrt{2} \lambda^{2}\right)\left(v_{1} U_{a 1}^{S} U_{12}^{P} U_{b 2}^{P}+v_{2} U_{a 2}^{S} U_{11}^{P} U_{b 1}^{P}\right) \\
& -\sqrt{2} i\left(\lambda k v_{1}+\lambda^{2} v_{2}\right) U_{a 2}^{S} U_{13}^{P} U_{b 3}^{P}-\sqrt{2} i\left(\lambda k v_{2}+\lambda^{2} v_{1}\right) U_{a 1}^{S} U_{13}^{P} U_{b 3}^{P} \\
& -\sqrt{2} i \lambda^{2} x \cdot U_{a 3}^{S}\left(U_{11}^{P} U_{b 1}^{P}+U_{12}^{P} U_{b 2}^{P}\right) \\
& -i\left(2 \sqrt{2} k^{2} x+\sqrt{2} k A_{k}\right) U_{a 3}^{S} U_{13}^{P} U_{b 3}^{P} \\
& +\sqrt{2} i \lambda k \cdot U_{a 3}^{S}\left[v_{1}\left(U_{12}^{P} U_{b 3}^{P}+U_{13}^{P} U_{b 2}^{P}\right)+v_{2}\left(U_{11}^{P} U_{b 3}^{P}+U_{13}^{P} U_{b 1}^{P}\right)\right] \\
& +i\left(\sqrt{2} \lambda k x-\frac{\lambda A_{\lambda}}{\sqrt{2}}\right) \times \\
& \times\left[U_{a 1}^{S}\left(U_{12}^{P} U_{b 3}^{P}+U_{13}^{P} U_{b 3}^{P}\right)+U_{a 2}^{S}\left(U_{11}^{P} U_{b 3}^{P}+U_{13}^{P} U_{b 1}^{P}\right)\right] \\
& -i\left(\sqrt{2} \lambda k x+\frac{\lambda A_{\lambda}}{\sqrt{2}}\right) U_{a 3}^{S}\left(U_{11}^{P} U_{b 2}^{P}+U_{12}^{P} U_{b 1}^{P}\right) . \\
& * M_{0}^{+} \cdot \delta M_{2}=\left(\frac{-g^{3}}{4}\right) \frac{i \pi^{2} A m_{W}}{(2 \pi)^{4}}\left(\sin \beta \cdot U_{11}^{P}+\cos \beta \cdot U_{12}^{P}\right)^{2} \text {. } \\
& \left(\cos \beta \cdot U_{\alpha 1}^{S}+\sin \beta \cdot U_{\alpha 2}^{S}\right) \text {. }
\end{aligned}
$$

$$
\begin{aligned}
& C_{0}(2)\left[-\left(m_{H}^{2}+3 m_{a_{1}}^{2}-m_{W}^{2}\right)+\frac{m_{H}^{2}-m_{a_{1}}^{2}-m_{W}^{2}}{m_{W}^{2}}\left(m_{H}^{2}-m_{a_{1}}^{2}\right)\right] \\
& \left\{+\frac{m_{W}^{2}-m_{H}^{2}+m_{a_{1}}^{2}}{m_{W}^{2}} C_{11}(2)\left[\begin{array}{l}
-\frac{\left(m_{H}^{2}+3 m_{a_{1}}^{2}-m_{W}^{2}\right)}{2} \\
+\frac{m_{H}^{2}-m_{a_{1}}^{2}-m_{W}^{2}}{2 m_{W}^{2}}\left(m_{H}^{2}-m_{a_{1}}^{2}\right)
\end{array}\right]\right. \\
& \left.-\frac{m_{W}^{2}-m_{H}^{2}+m_{a_{1}}^{2}}{m_{W}^{2}} C_{12}(2)\left[\begin{array}{l}
-\frac{3 m_{H}^{2}+m_{a_{1}}^{2}-m_{W}^{2}}{2} \\
+\frac{\left(m_{H}^{2}-m_{a_{1}}^{2}\right)}{2 m_{W}^{2}}\left(m_{H}^{2}+m_{W}^{2}-m_{a_{1}}^{2}\right)
\end{array}\right]\right]
\end{aligned}
$$

with: $C_{0}(2)=C_{0}\left(m_{S a}^{2}, m_{H}^{2}, m_{W}^{2}, m_{W}^{2}, m_{H}^{2}, m_{S a}^{2}\right)$,

$C_{11}(2)=C_{11}\left(m_{S a}^{2}, m_{H}^{2}, m_{W}^{2}, m_{W}^{2}, m_{H}^{2}, m_{S a}^{2}\right)$

and $C_{12}(2)=C_{12}\left(m_{S a}^{2}, m_{H}^{2}, m_{W}^{2}, m_{W}^{2}, m_{H}^{2}, m_{S a}^{2}\right)$ are the Pasarino-Vellman functions.

$$
\begin{gathered}
* M_{0}^{+} \delta M_{3}=i \pi^{2} \cdot B \cdot\left(\frac{-g^{3}}{4}\right) m_{W} \frac{1}{(2 \pi)^{D}}\left(\sin \beta \cdot U_{11}^{P}+\cos \beta \cdot U_{12}^{P}\right)^{+} \\
.\left(\sin \beta \cdot U_{b 1}^{P}+\cos \beta \cdot U_{b 2}^{P}\right) \cdot\left(\cos \beta \cdot U_{a 1}^{S}+\sin \beta \cdot U_{a 2}^{S}\right) \\
\left\{\begin{array}{l}
2 C_{0}(3)\left[-\frac{3 m_{H}^{2}+m_{a_{1}}^{2}-m_{W}^{2}}{2}+\frac{m_{H}^{2}+m_{W}^{2}-m_{a_{1}}^{2}}{2 m_{W}^{2}}\left(m_{H}^{2}-m_{a_{1}}^{2}\right)\right] \\
+\frac{m_{H}^{2}-m_{P b}^{2}+m_{W}^{2}}{m_{W}^{2}} C_{12}(3)\left[\begin{array}{l}
-\frac{m_{H}^{2}+3 m_{a_{1}}^{2}-m_{W}^{2}}{2} \\
\left.+\frac{m_{H}^{2}-m_{a_{1}}^{2}-m_{W}^{2}}{2 m_{W}^{2}}\left(m_{H}^{2}-m_{a_{1}}^{2}\right)\right]
\end{array}\right\}
\end{array}\right.
\end{gathered}
$$

with: $C_{0}(3)=C_{0}\left(m_{W}^{2}, m_{a_{1}}^{2}, m_{H}^{2}, m_{W}^{2}, m_{S a}^{2}, m_{P b}^{2}\right)$ and $C_{12}(3)=C_{12}\left(m_{W}^{2}, m_{a_{1}}^{2}, m_{H}^{2}, m_{W}^{2}, m_{S a}^{2}, m_{P b}^{2}\right)$.

To study the influence of the mass $m_{a 1}$ on the decay process $\mathrm{H}^{ \pm} \rightarrow \mathrm{W}^{ \pm} \mathrm{a}_{1}$, we use two set of parameters [7, $10]$ for programming numerical calculation.

The 1st parameter set: $\lambda=0.8 ; x=200 ; k=0.1 ; \quad m_{H}$ $=100 \mathrm{GeV} ; \tan \beta=3 ; \sin \alpha=-0.58 ; A_{k}=6 ; A_{\lambda}=486$. From the results obtained, we find that the influence of $\mathrm{m}_{\mathrm{a} 1}$ on the decay $\mathrm{H}^{ \pm} \rightarrow \mathrm{W}^{ \pm} \mathrm{a}_{1}$ is relatively significant (Figs. 2 and 3).

Specifically, the influence of $\mathrm{m}_{\mathrm{a}_{1}}$ on the decay width and on the lifetime of $\mathrm{H}^{ \pm}$in the decay $\mathrm{H}^{ \pm} \rightarrow \mathrm{W}^{ \pm} \mathrm{a}_{1}$ is relatively significant. When $\mathrm{m}_{\mathrm{a} 1}$ runs from 4 to 5 , it can influence $2.9 \%$ to the decay width and about $4 \%$ to the lifetime of $\mathrm{H}^{ \pm}$. With the $1 \mathrm{st}$ 


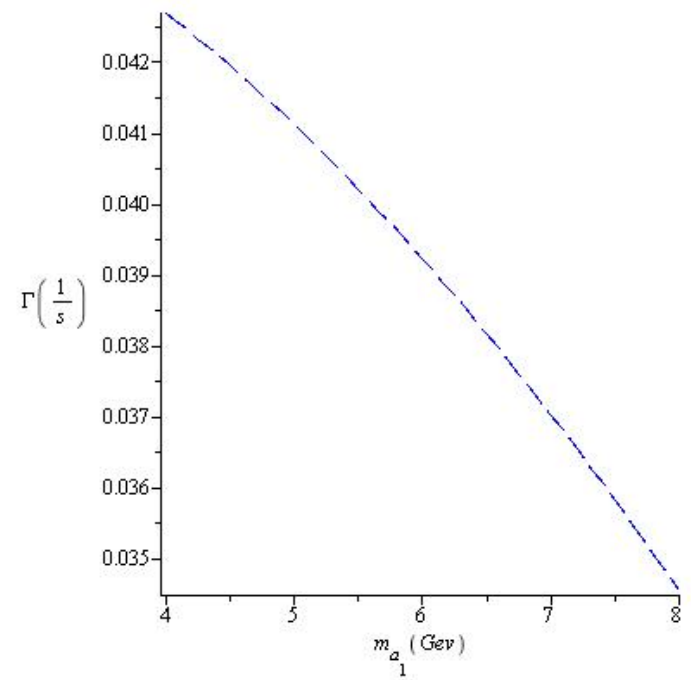

Fig. 2 The influence of $\mathbf{m}_{\mathrm{a} 1}$ on the decay width of the decay $\mathrm{H}^{ \pm} \rightarrow \mathrm{W}^{ \pm} \mathrm{a}_{1}$ with $\lambda=0.8 ; x=200 ; k=0.1 ; m_{H}=100 \mathrm{GeV}$; $\tan \beta=3 ; \sin \alpha=-0.58 ; A_{k}=6 ; A_{\lambda}=486$.

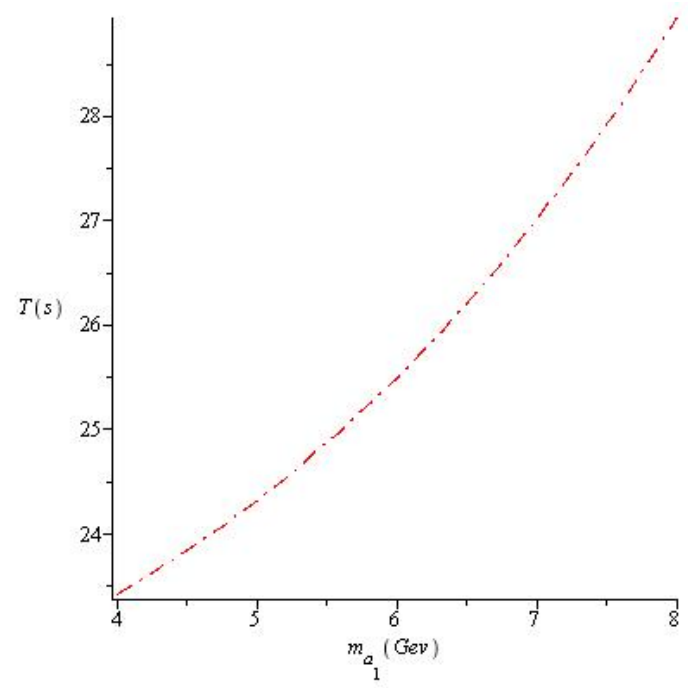

Fig. 3 The influence of $\mathbf{m}_{\mathrm{a} 1}$ on the lifetime of $\mathbf{H}^{ \pm}$of the decay $\mathrm{H}^{ \pm} \rightarrow \mathrm{W}^{ \pm} \mathrm{a}_{1}$ with $\lambda=0.8 ; x=200 ; k=0.1 ; m_{H}=100$ $\mathrm{GeV} ; \tan \beta=3 ; \sin \alpha=-0.58 ; A_{k}=6 ; A_{\lambda}=486$.

parameter set, we obtain the results of the decay width $\mathrm{H}^{ \pm} \rightarrow \mathrm{W}^{ \pm} \mathrm{a}_{1}$ at about $0.035-0.043(1 / \mathrm{s})$, and that of the lifetime of $\mathrm{H}^{ \pm}$at about 23.5-29 (s).

The 2nd parameter set: $\lambda=0.8 ; x=200 ; k=0.1$; $m_{H}=100 \mathrm{GeV} ; \tan \beta=10 ; \sin \alpha=-0.726 ; A_{k}=7 ; A_{\lambda}=$ 492. We obtain Figs. 4 and 5.

From the graph results with the 2nd parameter set, it can be seen that the influence of $m_{a 1}$ is also relatively significant, its contributions to the results are even more than that of the 1 st parameter set. When $m_{a 1}$ runs

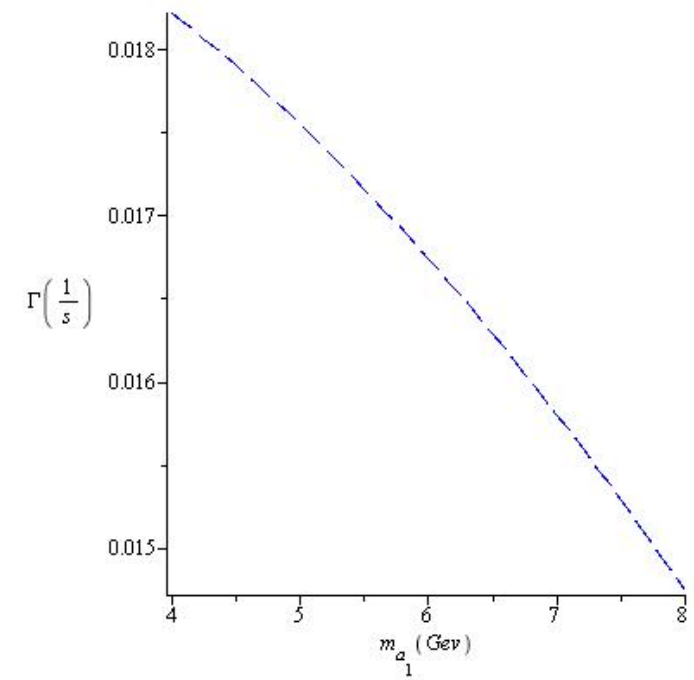

Fig. 4 The influence of $m_{a 1}$ on the decay width of the decay $\mathrm{H}^{ \pm} \rightarrow \mathrm{W}^{ \pm} \mathrm{a}_{1}$ with $\lambda=0.8 ; x=200 ; k=0.1 ; m_{H}=100 \mathrm{GeV}$; $\tan \beta=10 ; \sin \alpha=-0.726 ; A_{k}=6 ; A_{\lambda}=486$.

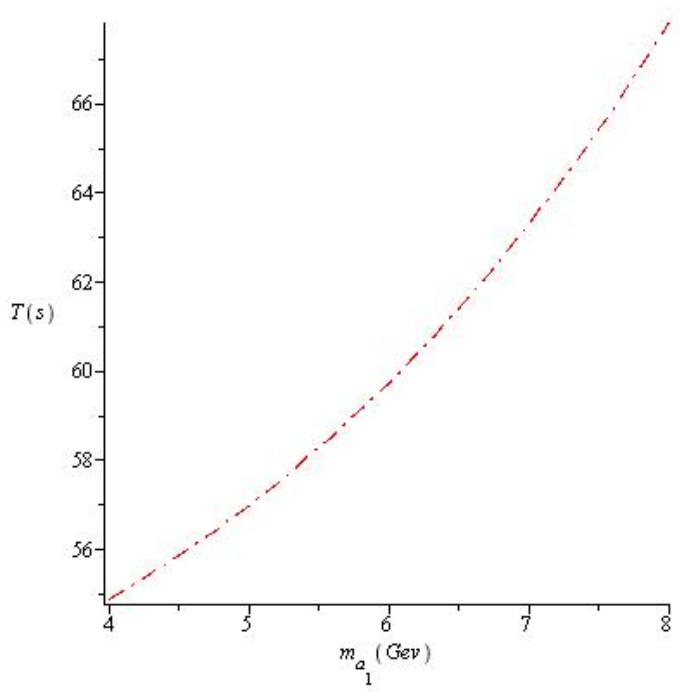

Fig. 5 The influence of $m_{a 1}$ on the lifetime of $\mathbf{H}^{ \pm}$of the decay $\mathrm{H}^{ \pm} \rightarrow \mathrm{W}^{ \pm} \mathrm{a}_{1}$ with $\lambda=0.8 ; x=200 ; k=0.1 ; m_{H}=100$ $\mathrm{GeV} ; \tan \beta=10 ; \sin \alpha=-0.726 ; A_{k}=6 ; A_{\lambda}=486$.

from 4 to 5 , it can influence $6.7 \%$ to the decay width and about $2.9 \%$ to the lifetime of $\mathrm{H}^{ \pm}$. With this parameter set, we obtain the value of the decay width $\mathrm{H}^{ \pm} \rightarrow \mathrm{W}^{ \pm} \mathrm{a}_{1}$ at about $0.015-0.018(1 / \mathrm{s})$, and that of the lifetime of $\mathrm{H}^{ \pm}$at about 55-61 (s).

\section{Conclusions}

In the NMSSM, a single superfield is added with complex scalar field components, this causes the appearance of seven Higgs in the NMSSM. In the 
search for Higgs boson basing on decayed products, we need to focus on some channels which have high branching ratios. In this paper, we study the dominant decay of charged Higgs: $\mathrm{H}^{ \pm} \rightarrow \mathrm{W}^{ \pm} \mathrm{a}_{1}$, it can be nearly $100 \%$, except when the mass of charged Higgs almost reaches to the mass of $\mathrm{W}^{+}$.

The above decay width is calculated to one loop vertex correction. The numerical results which assess the influences of $m_{a 1}$ are also shown in this paper. In this decay, the influence of $m_{a 1}$ is relatively significant. We need to pay attention to this element when studying theories as well as paying attention to the decay experiments of charged Higgs. The lifetime of $\mathrm{H}^{ \pm}$is relatively short, and this brings us the hope that we can find charged Higgs soon.

\section{Acknowledgments}

This research is supported by the National Foundation for Science and Technology Development (NAFOSTED) of Vietnam. Grant number: 103.03-2012.80.

\section{References}

[1] D. Radovan, F.G. John, Many light Higgs bosons in the next-to-minimal supersymmetric model, Phys. Rev. D 79 (5) (2009) 055014-055029.

[2] M.M. Almarashi, S. Moretti, Very light $C P$-odd Higgs bosons of the NMSSM at the LHC in $4 b$-quark final states, Phys. Rev. D 84 (1) (2011) 015014-015021.

[3] H.E. Haber, G.L. Kane, The search for supersymmetry: Probing physics beyond the standard model, Phys. Rep. 117 (2-4) (1985) 75-263.

[4] W. Bernrenther, M. Suzuki, The electric dipole moment of the electron, Rev. Mod. Phys. 63 (2) (1991) 313-340.

[5] M.M. Almarashi, S. Moretti, LHC signals of a heavy CP-even Higgs Boson in the NMSSM via decays into a $\mathrm{Z}$ and a light CP-odd Higgs state, Phys. Rev. D 85 (1) (2012) 017701-017705.

[6] A. Bartl, H. Eberl, K. Hidaka, S. Kraml, W. Majerotto, W. Porod, et al., SUSY-QCD corrections to stop and sbottom decays into W and Z bosons, Phys. Lett. B 419 (1998) 243-252.

[7] U. Ellwanger, Higgs bosons in the next-to-minimal supersymmetric standard model at the LHC, High Energy Physics -Phenomenology (2011).

[8] U. Ellwanger, C. Hugonie, A.M. Teixeira, The next-to-minimal supersymmetric standard model, Phys. Rept. 496 (1-2) (2010) 1-77.

[9] N.C. Cuong, Squark decays into $\mathrm{H}^{0}$ in the MSSM with complex parameters, Commu. Phys. 14 (4) (2004) 231-237.

[10] R. Dermisek, Light charged Higgs in the NMSSM, hep-ph/1012.3487 (2010). 


\title{
The Statistical Distribution for $\hat{g}$-Deformed Crystal Lattice Vibration for Generic Atomic String
}

\author{
Nguyen Thi Ha Loan \\ Department of Physics, Hanoi Pedagogical University No 2, Vinh Phuc 280000, Viet Nam
}

Revised: August 30, 2013 / Accepted: October 30, 2013 / Published: February 15, 2014.

\begin{abstract}
In this paper, a $\hat{g}$-deformation of crystal lattice vibration for generic atomic string is suggested. The expression for energy spectrum depends on $\hat{\mathrm{g}}$-deformed parameter have been obtained. The statistical distribution of $\hat{\mathrm{g}}$-deformed crystal lattice vibration is calculated and this result is very much different from there of q-deformed crystal lattice vibration.
\end{abstract}

Key words: Quantum, crystal, lattice, vibration.

\section{Introduction}

Over the last decades, there has been a lot of interest in the study of quantum groups and algebra. This is dictated by the point of view about their applicability in physical models [1-4]. They are relevant for a variety of diverse problems, such as the study of solutions to the Yang-Baxter equation, rational conformal field theory, two dimensional field theory with fractional statistics.

The deformed oscillators formalism plays a useful role in the study of the quantum optics, condensed matter physics, etc..

In this work, we construct a $\hat{\mathrm{g}}$-deformed crystal lattice vibration for a generic atomic string; this is a fundament for study of crystal lattice vibration, which would give a base for a new quantization procedure.

\section{2. $\hat{\mathrm{g}}$-Deformed Oscillator}

The Hamitonian of $\hat{g}$-deformed oscillator, system is given by $[5,6]$ :

$$
H=\frac{P^{2}}{2 m}+\frac{1}{2} m \omega^{2} x^{2}
$$

Corresponding author: Nguyen Thi Ha Loan, Ph.D., associate professor, research fields: deformedoscillators and applications in quantum physics. E-mail: hloansp2@gmail.com.
We define the creation (annihilation) operator $\mathrm{a}^{+}$(a) of $\hat{g}$-deformed oscillator as:

$$
\begin{aligned}
& a^{+}=\sqrt{\frac{m \omega}{2 \hbar}}\left(x-\frac{i}{m \omega} P\right) \\
& a=\sqrt{\frac{m \omega}{2 \hbar}}\left(x+\frac{i}{m \omega} P\right)
\end{aligned}
$$

Hence, the coordinate and momentum operators can be expressed in terms of creation and annihilation operators $\mathrm{a}^{+}$, a as:

$$
\begin{aligned}
& x=\sqrt{\frac{\hbar}{2 m \omega}}\left(a+a^{+}\right) \\
& p=-i \sqrt{\frac{\hbar m \omega}{2}}\left(a-a^{+}\right)
\end{aligned}
$$

Using Eqs. (3) and (1), we obtain:

$$
H=\frac{\hbar \omega}{2}\left(a \cdot a^{+}+a^{+} \cdot a\right)
$$

The creation (annihilation) operator $\mathrm{a}^{+}$.(a) and the number operator $\mathrm{N}$ satisfies the commutation relations [2]:

$$
\begin{aligned}
& a \cdot a^{+}-\hat{g} \cdot a^{+} \cdot a=1 \\
& {[\hat{g}, a]=\left[\hat{g}, a^{+}\right]=0} \\
& \hat{g}=\hat{g}^{+}, \quad \hat{g}^{2}=1 \\
& {[N, a]=-a} \\
& \left.N, a^{+}\right]=a^{+}
\end{aligned}
$$

The Fock space basics can be introduced:

$$
|n\rangle=\frac{\left(a^{+}\right)^{n}}{\sqrt{[n]_{g} !}}|0\rangle
$$


where, $|0\rangle$ is the ground state satisfying the conditions:

$$
\begin{aligned}
& a|0\rangle=0 \\
& N|n\rangle=n|n\rangle \\
& a^{+}|n\rangle=\sqrt{[n+1]_{g}}|n+1\rangle \\
& a|n\rangle=\sqrt{[n]_{g}|n-1\rangle}
\end{aligned}
$$

The energy spectrum of the $\hat{\mathrm{g}}$-deformed oscillator is defined by the equation:

$$
H|n\rangle=E_{n}|n\rangle
$$

where

$$
\begin{aligned}
& E_{n}=\frac{\hbar \omega}{2}\left\{[n+1]_{g}+[n]_{g}\right\} \\
& {[n]_{g}=\frac{1-\hat{g}^{n}}{1-\hat{g}}}
\end{aligned}
$$

\section{The g-Deformed Crystal Lattice Vibration for a Generic Atomic String}

The Hamitonian of generic atomic string can be expressed as:

$$
H=\sum_{k}^{1}\left(\frac{1}{2 M} P_{-k} P_{k}+\frac{1}{2} M \omega^{2} u_{-k} u_{k}\right)
$$

where, $u_{k}$ is genezalized coordinate and $P_{k}$ is corresponding momentum of generic atomic string. $\sum_{\mathrm{K}}^{\mathrm{i}}$ is total of $\mathrm{k}$, which is waved vector in the first Brillouin area.

The creation and annihilation operators corresponding to wave vector $\mathrm{k}$ have the forms:

$$
\begin{aligned}
& a_{k}=\frac{1}{\sqrt{2 \hbar \omega}}\left(\sqrt{M} \omega u_{k}+\frac{i}{\sqrt{M}} P_{k}\right) \\
& a_{k}^{+}=\frac{1}{\sqrt{2 \hbar \omega}}\left(\sqrt{M} \omega u_{-k}-\frac{i}{\sqrt{M}} P_{-k}\right)
\end{aligned}
$$

The coordinate and momentum operators can be expressed in terms of creation and annihilation operators $a_{-k}^{+}, a_{k}$ as:

$$
\begin{aligned}
& u_{k}=\sqrt{\frac{\hbar}{2 M \omega}}\left(a_{k}+a_{-k}^{+}\right) \\
& P_{k}=-i \sqrt{\frac{\hbar M \omega}{2}}\left(a_{k}-a_{-k}^{+}\right)
\end{aligned}
$$

Using Eqs. (12) and (10), we obtain:

$$
H=\sum_{k}^{1} \frac{\hbar \omega}{2}\left(a_{-k} a_{-k}^{+}+a_{k}^{+} a_{k}\right)
$$

The oscillator number operator and the oscillator creation, annihilation operator satisfies the following commutation relations:

$$
\begin{aligned}
& a_{k} a_{-k^{\prime}}^{+}-\hat{g} a_{-k^{\prime}}^{+} a_{k}=\delta_{k,-k} \\
& a_{k}^{+} a_{-k^{\prime}}^{+}-\hat{g} a_{-k^{\prime}}^{+} a_{k}^{+}=0 \\
& {\left[\hat{g}, a_{k}\right]=\left[\hat{g}, a_{-k}^{+}\right]=0} \\
& {\left[N_{k}, a_{-k^{+}}^{+}\right]=a_{k}^{+} \delta_{k,-k^{\prime}}} \\
& {\left[N_{k}, a_{k^{\prime}}\right]=-a_{k} \delta_{k, k^{\prime}}}
\end{aligned}
$$

The $\hat{\mathrm{g}}$-deformed state vectors in Fock space are:

$$
|n\rangle=\left|n_{k}, n_{-k}\right\rangle=\frac{\left(a_{k}^{+}\right)^{n_{k}}\left(a_{-k}^{+}\right)^{n_{-k}}}{\sqrt{\left[n_{k}\right]_{g} !\left[n_{-k}\right]_{g} !}}|0\rangle
$$

where, $|0\rangle$ is the ground state and:

$$
\begin{gathered}
{\left[n_{k}\right]_{g}=\frac{1-\hat{g}^{n_{k}}}{1-\hat{g}}} \\
{\left[n_{k}\right]_{g} !=\left[n_{k}\right]_{g}\left[n_{k}-1\right]_{g} \ldots[1]_{g}}
\end{gathered}
$$

The eigenstates $|n\rangle$ of the oscillator number operators is as follow:

$$
N_{k}|n\rangle=n_{k}|n\rangle
$$

From Eq. (14), it can be seen that the action of operators $a_{k}^{+}$and ${ }^{a_{k}}$ on the eigenstates $|n\rangle$ gives:

$$
\begin{aligned}
& a_{k}^{+}|n\rangle=\sqrt{\left[n_{k}+1\right]_{g}}\left|n_{k}+1, n_{-k}\right\rangle \\
& a_{k}|n\rangle=\sqrt{\left[n_{k}\right]_{g}}\left|n_{k}-1, n_{-k}\right\rangle \\
& a_{-k}^{+}|n\rangle=\hat{g}^{n_{k}} \sqrt{\left[n_{-k}+1\right]_{g}}\left|n_{k}, n_{-k}+1\right\rangle \\
& a_{-k}|n\rangle=\hat{g}^{n_{k}} \sqrt{\left[n_{-k}\right]_{g}}\left|n_{k}, n_{-k}-1\right\rangle
\end{aligned}
$$

The energy spectrum of $\hat{g}$-deformed crystal lattice vibration for a generic atomic string is given by:

$$
H|n\rangle=E_{n}|n\rangle
$$

where,

$$
E_{n}=\sum_{k}^{1} \frac{\hbar \omega}{2}\left\{\left[n_{-k}+1\right]_{g}+\left[n_{k}\right]_{g}\right\}
$$

If the Hamitonian (13) of the crystal lattice vibration for a generic atomic string in the $\hat{g}$-deformed quantum theory can be expressed in terms of creation annihilation operators $a_{k}^{+}, a_{k}$, which are defined to satisfy the commutation relations (14), the crystal lattice vibration are then considered as particle system and its energy spectrum depended on deformed 
parameters according to Eq. (21).

\section{The $\hat{g}$-Deformed Green Function}

The g-deformed Green function is defined as the statistical distribution of $a_{k}^{+} a_{k}$ :

$$
\left\langle a_{k}^{+} a_{k}\right\rangle=\frac{1}{Z} \operatorname{tr}\left(e^{-\beta \omega N_{k}} a_{k}^{+} a_{k}\right)
$$

where, $Z$ is the partition function:

$$
\mathrm{Z}=\operatorname{Tr}\left(e^{-\beta \omega N_{k}}\right)=\sum_{n=0}^{\infty}\left\langle n\left|e^{-\beta \omega N_{k}}\right| n\right\rangle
$$

which determines the thermodynamic properties of the system, $\beta=1 / k T, \omega$ being one particle-oscillator energy. The trace must be taken over a complete set of states.

The caculations based on the Eq. (15) give the following results:

$$
a_{k}^{+} a_{k}=\frac{1}{e^{\beta \omega}-\hat{g}}
$$

\section{Conclusions}

The present construction a statistical distribution for $\hat{g}$-deformed crystal lattice vibration for generic atomic string is an example of use deformed oscillators formalism to study of condensed matter physics, this is a fundament for study of crystal lattice vibration, which would give a base for a new quantization procedure. Further the next step is to extend the present investigations to the case of other physical systems.

\section{References}

[1] N.T.H. Loan, Deformed oscillator and their statistics, Com. In. Phys. 6 (2) (1996) 18-22.

[2] H.H. Bang, C.T.V. Ba, The g-deformed $\mathrm{N}=2$ super symmetric algebra, Com. In. Phys. 3 (2002) 143-146.

[3] N.V. Hieu, N.B. An, Theoretical Fundament of Quantum Physics, Hanoi National University Publisher, 2003.

[4] G. Ju, J. Tao, Z. Liu, M. Wang, The eigenvectors of q-deformed creation operation $a_{q}^{+}$and their properties, Mod. Phys. Lett. A 10 (8) (1995) 669-675.

[5] T. Kobayashi, T. Suzuki, Quantum mechanics with q-deformed commutations and periodic variables, J. Phys. A 26 (21) (1993) 6055-6066.

[6] A.S. Davyov, Quantum mechanics, Translate by Đặng Quang Khang, Hanoi Science and Technics Publisher, 1972. 


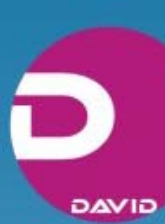

Journal of Physical Science and Application

Volume 4, Number 2, February 2014

David Publishing Company

240 Nagle Avenue \#15C, New York, NY 10034, USA

Tel: 1-323-984-7526, 323-410-1082; Fax: 1-323-984-7374, 323-908-0457

http://www.davidpublishing.com, www.davidpublishing.org

physical@davidpublishing.org, physics.david@yahoo.com 